\section{New Theory}

ISSN: 2149-1402
36 (2021) 18-38

Journal of New Theory

https://dergipark.org.tr/en/pub/jnt

Open Access

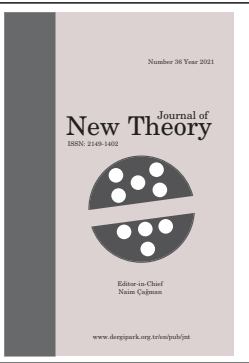

\title{
Theory of Generalized Sets in Generalized Topological Spaces
}

\author{
Mohammad Irshad Khodabocus ${ }^{1}$ (D) , Noor-Ul-Hacq Sookia ${ }^{2}$ (D)
}

\author{
Article History \\ Received: 13 Mar 2021 \\ Accepted: 20 Sep 2021 \\ Published: 30 Sep 2021 \\ 10.53570/jnt.896345 \\ Research Article
}

\begin{abstract}
Several specific types of generalized sets (briefly, $\mathfrak{g}-\mathfrak{T}_{\mathfrak{g}}$-sets) in generalized topological spaces (briefly, $\mathcal{T}_{\mathfrak{g}}$-spaces) have been defined and investigated for various purposes from time to time in the literature of $\mathcal{T}_{\mathfrak{g}}$-spaces. Our recent research in the field of a new class of $\mathfrak{g}$ - $\mathfrak{T}_{\mathfrak{g}}$-sets in $\mathcal{T}_{\mathfrak{g}}$-spaces is reported herein as a starting point for more generalized classes. It is shown that the class of $\mathfrak{g}$ - $\mathfrak{T}_{\mathfrak{g}}$-sets is a superclass of those whose elements are called open, closed, semi-open, semi-closed, pre-open, pre-closed, semi-pre-open, and semi-pre-closed sets in a $\mathcal{T}_{\mathfrak{g}}$-space. A subclass of the $\mathcal{T}_{\mathfrak{g}}$-subspace corresponds to the class of $\mathfrak{g}$ - $\mathfrak{T}_{\mathfrak{g}}$-sets of a $\mathcal{T}_{\mathfrak{g}}$-space. A class of $\mathfrak{g}-\mathfrak{T}_{\mathfrak{g}}$-sets of the Cartesian product of these $\mathcal{T}_{\mathfrak{g}}$-spaces corresponds to the Cartesian product of a finite number of classes of $\mathfrak{g}-\mathfrak{T}_{\mathfrak{g}}$-sets, each of which belongs to a $\mathcal{T}_{\mathfrak{g}}$-space. Diagrams establish the various relationships amongst the classes presented here and in the literature, and an ad hoc application supports the overall theory.
\end{abstract}

Keywords - Generalized topology, generalized topological space, generalized operations, generalized open sets, generalized closed sets

Mathematics Subject Classification (2020) - 54A05, 54B05

\section{Introduction}

Just as the notion of $\mathcal{T}$-set (open or closed set relative to ordinary topology) is fundamental and indispensable in the study of $\mathfrak{T}$-sets in $\mathcal{T}$-spaces (arbitrary sets in ordinary topological spaces) and in the formulation of the concept of $\mathfrak{g}$ - $\mathcal{T}$-set (generalized $\mathcal{T}$-open or $\mathcal{T}$-closed set relative to ordinary topology) in the study of $\mathfrak{g}$ - $\mathfrak{T}$-sets in $\mathcal{T}$-spaces (generalized sets in ordinary topological spaces) [1-6], so is the notion of $\mathcal{T}_{\mathfrak{g}}$-set (open or closed set relative to generalized topology) in the study of $\mathfrak{T}_{\mathfrak{g}}$-sets in $\mathcal{T}_{\mathfrak{g}}$-spaces (arbitrary sets in generalized topological spaces) and in the formulation of the concept of $\mathfrak{g}$ - $\mathcal{T}_{\mathfrak{g}}$-set (generalized $\mathcal{T}_{\mathfrak{g}}$-open or $\mathcal{T}_{\mathfrak{g}}$-closed set relative to generalized topology) in the study of $\mathfrak{g}$ - $\mathfrak{T}_{\mathfrak{g}}$ sets in $\mathcal{T}_{\mathfrak{g}}$-spaces (generalized sets in generalized topological spaces) [7]. Thus, the $\mathfrak{g}$-topology maps $\mathcal{T}_{\mathfrak{g}}: \mathcal{P}(\Omega) \longrightarrow \mathcal{P}(\Omega)$ from the power set $\mathcal{P}(\Omega)$ of $\Omega$ into itself, thereby inducing $\mathfrak{g}$-topologies on the underlying set $\Omega$, are classes of distinguished open subsets of a $\mathcal{T}$-space which are not $\mathcal{T}$-open sets but are $\mathcal{T}_{\mathfrak{g}}$-open sets which are related to the families of $\mathfrak{g}$ - $\mathcal{T}$-open sets $[8,9]$. Examples of $\mathfrak{g}$ - $\mathfrak{T}$-sets in $\mathcal{T}$-spaces are $\alpha$-open and $\alpha$-closed sets [10], $\beta$-open sets [11], and $\gamma$-open sets [12]. Examples of $\mathfrak{g}$ - $\mathfrak{T}_{\mathfrak{g}}$-sets in $\mathcal{T}_{\mathfrak{g}}$-spaces are $\Delta_{\mu}$-sets and $\nabla_{\mu}$-sets [13], $\omega$-open sets [2], and $\theta$-sets [14]. From these $\alpha$, $\beta, \gamma$-sets and, $\Delta_{\mu}, \nabla_{\mu}, \omega, \theta$-sets, the theories of $\mathfrak{g}$-T-sets and $\mathfrak{g}$ - $\mathfrak{T}_{\mathfrak{g}}$-sets then appear to be subjects of primary interest.

To the best of our knowledge, the theory of $\mathfrak{g}$-T-sets is well-known and that of $\mathfrak{g}$ - $\mathfrak{T}_{\mathfrak{g}}$-sets lessknown. The earliest works on the theory of $\mathfrak{g}$-T-sets are those of Levine [15,16], Njåstad [10], and

\footnotetext{
${ }^{1}$ ikhodabo@gmail.com (Corresponding Author); ${ }^{2}$ sookian@uom.ac.mu

${ }^{1}$ Department of Emerging Technologies, Faculty of Sustainable Development and Engineering, Université des Mascareignes, Rose Hill Campus, Mauritius

${ }^{2,2}$ Department of Mathematics, Faculty of Science, University of Mauritius, Réduit, Mauritius
} 
Császár [14,17-20], and the latest works on the theory of $\mathfrak{g}$-T-sets are those of Rajeshwari et al. [21], Jeyanthi et al. [3,13], Ghour et al. [2], and Tyagi et al. [6], among others. Levine [16] introduced and investigated the weaker forms of open sets, Njåstad [10] introduced and investigated the structures of some classes of more or less nearly open sets, and Császár [20] introduced the notion of g-topologies; [21] introduced the weaker forms of closed sets and studied some of their characterizations, Jeyanthi et al. [3] gave a unified framework for the study of several types of $\mathfrak{g}-\mathfrak{T}_{\mathfrak{g}}$-sets, Ghour et al. [2] extended the notion of a type of $\mathfrak{g}$ - $\mathfrak{T}$-sets in a $\mathcal{T}$-space to its analogue in a $\mathcal{T}_{\mathfrak{g}}$-space, and Tyagi et al. [6] introduced and investigated several types of $\mathfrak{g}$ - $\mathfrak{T}_{\mathfrak{g}}$-sets in $\mathcal{T}_{\mathfrak{g}}$-spaces.

Several other specific classes of $\mathfrak{g}-\mathfrak{T}, \mathfrak{g}-\mathfrak{T}_{\mathfrak{g}}$-sets have been defined and investigated by other authors for various purposes from time to time in the literature of $\mathcal{T}, \mathcal{T}_{\mathfrak{g}}$-spaces $[9,22-38]$. The fruitfulness of all these references have made significant contributions to the theory of $\mathcal{T}, \mathcal{T}_{\mathfrak{g}}$-spaces, among others.

In this paper, we will show how further contributions can be added to the field in a unified way. The rest of this paper is structured in this manner: In Section 2, preliminary notions are described in Subsection 2.1 and the main results of the theory of $\mathfrak{g}-\mathfrak{T}_{\mathfrak{g}}$-sets in $\mathcal{T}_{\mathfrak{g}}$-spaces are reported in Section 3. In Section 4, the establishment of the various relationships between the classes of $\mathfrak{T}_{\mathfrak{g}}$-open and $\mathfrak{T}_{\mathfrak{g}}$-closed sets and the classes of $\mathfrak{g}$ - $\mathfrak{T}_{\mathfrak{g}}$-open and $\mathfrak{g}$ - $\mathfrak{T}_{\mathfrak{g}}$-closed sets in the $\mathcal{T}_{\mathfrak{g}}$-space $\mathfrak{T}_{\mathfrak{g}}$ are discussed and illustrated through diagrams in Subsection 4.1. To support the work, a nice application, concentrating on fundamental concepts from the standpoint of the theory of $\mathfrak{g}-\mathfrak{T}_{\mathfrak{g}}$-sets is presented in Subsection 4.2. Finally, Subsection 5 provides concluding remarks and future directions of the theory of $\mathfrak{g}$ - $\mathfrak{T}_{\mathfrak{g}}$-sets in $\mathcal{T}_{\mathfrak{g}^{-}}$-spaces.

\section{Theory}

\subsection{Preliminaries}

Our discussion starts by recalling a carefully chosen set of terms used in this study [39]. Throughout this manuscript, the structures $\mathfrak{T}=(\Omega, \mathcal{T})$ and $\mathfrak{T}_{\mathfrak{g}}=\left(\Omega, \mathcal{T}_{\mathfrak{g}}\right)$, respectively, are called ordinary and generalized topological spaces (briefly, $\mathcal{T}$-space and $\mathcal{T}_{\mathfrak{g}}$-space). The symbols $\mathcal{T}$ and $\mathcal{T}_{\mathfrak{g}}$, respectively, are called ordinary topology and generalized topology (briefly, topology and $\mathfrak{g}$-topology). Subsets of $\mathfrak{T}$ and $\mathfrak{T}_{\mathfrak{g}}$, respectively, are called $\mathfrak{T}_{\text {-sets and }} \mathfrak{T}_{\mathfrak{g}}$-sets; subsets of $\mathcal{T}$ and $\mathcal{T}_{\mathfrak{g}}$, respectively, are called $\mathcal{T}$-open and $\mathcal{T}_{\mathfrak{g}}$-open sets, and their complements are called $\mathcal{T}$-closed and $\mathcal{T}_{\mathfrak{g}}$-closed sets. Generalizations of $\mathfrak{T}$-sets, $\mathcal{T}$-open and $\mathcal{T}$-closed sets in $\mathcal{T}$, respectively, are called $\mathfrak{g}$-T-sets, $\mathfrak{g}$ - $\mathcal{T}$-open and $\mathfrak{g}$ - $\mathcal{T}$-closed sets; generalizations of $\mathfrak{T}_{\mathfrak{g}}$-sets, $\mathcal{T}_{\mathfrak{g}}$-open and $\mathcal{T}_{\mathfrak{g}}$-closed sets in $\mathcal{T}_{\mathfrak{g}}$, respectively, are called $\mathfrak{g}$ - $\mathfrak{T}_{\mathfrak{g}}$-sets, $\mathfrak{g}$ - $\mathcal{T}_{\mathfrak{g}}$-open and $\mathfrak{g}-\mathcal{T}_{\mathfrak{g}}$-closed sets; $\mathfrak{U}$ stands for the universe of discourse, fixed within the framework of the theory of $\mathfrak{g}$ - $\mathfrak{T}_{\mathfrak{g}}$-sets and containing as elements all sets $\left(\Omega, \Gamma\right.$-sets; $\mathcal{T}, \mathfrak{g}-\mathcal{T}, \mathfrak{T}, \mathfrak{g}$-T-sets; $\mathcal{T}_{\mathfrak{g}}, \mathfrak{g}$ - $\mathcal{T}_{\mathfrak{g}}, \mathfrak{T}_{\mathfrak{g}}, \mathfrak{g}$ - $\mathfrak{T}_{\mathfrak{g}}$-sets $)$ considered in this theory, and $I_{n}^{0}:=\left\{\nu \in \mathbb{N}^{0}: \nu \leq n\right\}$; index sets $I_{\infty}^{0}, I_{n}^{*}, I_{\infty}^{*}$ are defined similarly. A set $\Gamma \subset \mathfrak{U}$ is a subset of the set $\Omega \subset \mathfrak{U}$ and, for some $\mathcal{T}_{\mathfrak{g}}$-open set $\mathcal{O}_{\mathfrak{g}} \in \mathcal{T} \cup \mathfrak{g}-\mathcal{T} \cup \mathcal{T}_{\mathfrak{g}} \cup \mathfrak{g}-\mathcal{T}_{\mathfrak{g}}$, these implications hold:

$$
\mathcal{O}_{\mathfrak{g}} \in \mathcal{T} \Rightarrow \mathcal{O}_{\mathfrak{g}} \in \mathfrak{g}-\mathcal{T} \Rightarrow \mathcal{O}_{\mathfrak{g}} \in \mathcal{T}_{\mathfrak{g}} \Rightarrow \mathcal{O}_{\mathfrak{g}} \in \mathfrak{g}-\mathcal{T}_{\mathfrak{g}} \Rightarrow \mathcal{O}_{\mathfrak{g}} \subset \Omega \subset \mathfrak{U}
$$

In a natural way, a monotonic map $\mathcal{T}_{\mathfrak{g}}: \mathcal{P}(\Omega) \longrightarrow \mathcal{P}(\Omega)$ from the power set $\mathcal{P}(\Omega)$ of $\Omega$ into itself can be associated to a given mapping $\pi_{\mathfrak{g}}: \Omega \longrightarrow \Omega$, thereby inducing a $\mathfrak{g}$-topology $\mathcal{T}_{\mathfrak{g}} \subset \mathcal{P}(\Omega)$ on the underlying set $\Omega$ [9]. Therefore, the definition of a $\mathcal{T}_{\mathfrak{g}}$-space can be presented in a nice way. Thus, retaining the axioms to be satisfied by its $\mathfrak{g}$-topology [33], and assuming no separation axioms, unless otherwise stated, the following definition is suggestive:

Definition 2.1 $\left(\mathcal{T}_{\mathfrak{g}}\right.$-Space [39]). Let $\Omega \subset \mathfrak{U}$ be a given set and let $\mathcal{P}(\Omega):=\left\{\mathcal{O}_{\mathfrak{g}, \nu}: \mathcal{O}_{\mathfrak{g}, \nu} \subseteq \Omega\right\}$ be the family of all subsets $\mathcal{O}_{\mathfrak{g}, 1}, \mathcal{O}_{\mathfrak{g}, 2}, \ldots$, of $\Omega$. Then, every one-valued map of the type $\mathcal{T}_{\mathfrak{g}}: \mathcal{P}(\Omega) \longrightarrow \mathcal{P}(\Omega)$ satisfying the following axioms:
i. $\mathcal{T}_{\mathfrak{g}}(\emptyset)=\emptyset$
ii. $\mathcal{T}_{\mathfrak{g}}\left(\mathcal{O}_{\mathfrak{g}}\right) \subseteq \mathcal{O}_{\mathfrak{g}}$ 
iii. $\mathcal{T}_{\mathfrak{g}}\left(\bigcup_{\nu \in I_{\infty}^{*}} \mathcal{O}_{\mathfrak{g}, \nu}\right)=\bigcup_{\nu \in I_{\infty}^{*}} \mathcal{T}_{\mathfrak{g}}\left(\mathcal{O}_{\mathfrak{g}, \nu}\right)$

is called a " $\mathfrak{g}$-topology on $\Omega$," and the structure $\mathfrak{T}_{\mathfrak{g}}:=\left(\Omega, \mathcal{T}_{\mathfrak{g}}\right)$ is called a "T $\mathcal{T}_{\mathfrak{g}}$-space."

In Definition 2.1, by Ax. i., Ax. ii., and Ax. iii., respectively, are meant that the unary operation $\mathcal{T}_{\mathfrak{g}}: \mathcal{P}(\Omega) \longrightarrow \mathcal{P}(\Omega)$ preserves nullary union, is contracting and preserves binary union. Any element $\mathcal{O}_{\mathfrak{g}} \in \mathcal{T}_{\mathfrak{g}}(\Omega)$ of the $\mathcal{T}_{\mathfrak{g}}$-space $\mathfrak{T}_{\mathfrak{g}}$ is called a $\mathcal{T}_{\mathfrak{g}}$-open set and its complement element $\complement\left(\mathcal{O}_{\mathfrak{g}}\right)=\mathcal{K}_{\mathfrak{g}} \notin \mathcal{T}_{\mathfrak{g}}(\Omega)$ is called a $\mathcal{T}_{\mathfrak{g}}$-closed set. If there exists a $\nu \in I_{\infty}^{*}$ such that $\mathcal{O}_{\mathfrak{g}, \nu}=\Omega$, then $\mathfrak{T}_{\mathfrak{g}}$ is called a strong $\mathcal{T}_{\mathfrak{g}}$ space $[9,19]$. Moreover, if the relation $\mathcal{T}_{\mathfrak{g}}\left(\bigcap_{\nu \in I_{n}^{*}} \mathcal{O}_{\mathfrak{g}, \nu}\right)=\bigcap_{\nu \in I_{n}^{*}} \mathcal{T}_{\mathfrak{g}}\left(\mathcal{O}_{\mathfrak{g}, \nu}\right)$ holds for any index set $I_{n}^{*} \subset I_{\infty}^{*}$ such that $n<\infty$, then $\mathfrak{T}_{\mathfrak{g}}$ is called a quasi $\mathcal{T}_{\mathfrak{g}}$-space [17].

Definition $2.2\left(\mathfrak{g}\right.$-Closure, $\mathfrak{g}$-Interior Operators [39]). Let $\mathfrak{T}_{\mathfrak{g}}$ be a $\mathcal{T}_{\mathfrak{g}}$-space on the set $\Omega \subset \mathfrak{U}$ with a $\mathfrak{g}$-topology $\mathcal{T}_{\mathfrak{g}}: \mathcal{P}(\Omega) \longrightarrow \mathcal{P}(\Omega)$. Then,

$i$. The operator $\mathrm{cl}_{\mathfrak{g}}: \mathcal{P}(\Omega) \longrightarrow \mathcal{P}(\Omega)$ carrying each $\mathfrak{T}_{\mathfrak{g}}$-set $\mathcal{S}_{\mathfrak{g}} \subset \mathfrak{T}_{\mathfrak{g}}$ into its closure $\mathrm{cl}_{\mathfrak{g}}\left(\mathcal{S}_{\mathfrak{g}}\right)=$ $\mathfrak{T}_{\mathfrak{g}} \backslash \operatorname{int}_{\mathfrak{g}}\left(\mathfrak{T}_{\mathfrak{g}} \backslash \mathcal{S}_{\mathfrak{g}}\right) \subset \mathfrak{T}_{\mathfrak{g}}$ is called a " $\mathfrak{g}$-closure operator."

ii. The operator $\operatorname{int}_{\mathfrak{g}}: \mathcal{P}(\Omega) \longrightarrow \mathcal{P}(\Omega)$ carrying each $\mathfrak{T}_{\mathfrak{g}}$-set $\mathcal{S}_{\mathfrak{g}} \subset \mathfrak{T}_{\mathfrak{g}}$ into its interior $\operatorname{int}_{\mathfrak{g}}\left(\mathcal{S}_{\mathfrak{g}}\right)=$ $\mathfrak{T}_{\mathfrak{g}} \backslash \mathrm{cl}_{\mathfrak{g}}\left(\mathfrak{T}_{\mathfrak{g}} \backslash \mathcal{S}_{\mathfrak{g}}\right) \subset \mathfrak{T}_{\mathfrak{g}}$ is called a " $\mathfrak{g}$-interior operator."

By convention, we let $\mathcal{T}_{\mathfrak{g}}(\Omega)$ and $\neg \mathcal{T}_{\mathfrak{g}}(\Omega)$, respectively, stand for the classes of all $\mathcal{T}_{\mathfrak{g}}$-open and $\mathcal{T}_{\mathfrak{g}}$-closed sets relative to the $\mathfrak{g}$-topology $\mathcal{T}_{\mathfrak{g}}$. Their proper definitions are contained below.

Definition 2.3 (Classes: $\mathcal{T}_{\mathfrak{g}}$-Open, $\mathcal{T}_{\mathfrak{g}}$-Closed Sets [39]). Let $\mathfrak{T}_{\mathfrak{g}}$ be a $\mathcal{T}_{\mathfrak{g}}$-space, let $\complement: \mathcal{P}(\Omega) \longrightarrow \mathcal{P}(\Omega)$ denotes the absolute complement with respect to the underlying set $\Omega \subset \mathfrak{U}$, and let $\mathcal{S}_{\mathfrak{g}} \subset \mathfrak{T}_{\mathfrak{g}}$ be any $\mathfrak{T}_{\mathfrak{g}}$-set. The classes

$$
\mathcal{T}_{\mathfrak{g}}(\Omega):=\left\{\mathcal{O}_{\mathfrak{g}} \subset \mathfrak{T}_{\mathfrak{g}}: \mathcal{O}_{\mathfrak{g}} \in \mathcal{T}_{\mathfrak{g}}\right\} \quad \text { and } \quad \neg \mathcal{T}_{\mathfrak{g}}(\Omega):=\left\{\mathcal{K}_{\mathfrak{g}} \subset \mathfrak{T}_{\mathfrak{g}}: \complement\left(\mathcal{K}_{\mathfrak{g}}\right) \in \mathcal{T}_{\mathfrak{g}}\right\}
$$

respectively, denote the classes of all $\mathcal{T}_{\mathfrak{g}}$-open and $\mathcal{T}_{\mathfrak{g}}$-closed sets relative to the $\mathfrak{g}$-topology $\mathcal{T}_{\mathfrak{g}}$, and the classes

$$
\mathrm{C}_{\mathcal{T}_{\mathfrak{g}}}^{\text {sub }}\left[\mathcal{S}_{\mathfrak{g}}\right]:=\left\{\mathcal{O}_{\mathfrak{g}} \in \mathcal{T}_{\mathfrak{g}}: \mathcal{O}_{\mathfrak{g}} \subseteq \mathcal{S}_{\mathfrak{g}}\right\} \quad \text { and } \quad \mathrm{C}_{\neg \mathcal{T}_{\mathfrak{g}}}^{\text {sup }}\left[\mathcal{S}_{\mathfrak{g}}\right]:=\left\{\mathcal{K}_{\mathfrak{g}} \in \neg \mathcal{T}_{\mathfrak{g}}: \mathcal{K}_{\mathfrak{g}} \supseteq \mathcal{S}_{\mathfrak{g}}\right\}
$$

respectively, denote the classes of $\mathcal{T}_{\mathfrak{g}}$-open subsets and $\mathcal{T}_{\mathfrak{g}}$-closed supersets (complements of the $\mathcal{T}_{\mathfrak{g}}$-open subsets) of the $\mathfrak{T}_{\mathfrak{g}}$-set $\mathcal{S}_{\mathfrak{g}} \subset \mathfrak{T}_{\mathfrak{g}}$ relative to the $\mathfrak{g}$-topology $\mathcal{T}_{\mathfrak{g}}$.

That $\mathrm{C}_{\mathcal{T}_{\mathfrak{g}}}^{\text {sub }}\left[\mathcal{S}_{\mathfrak{g}}\right] \subseteq \mathcal{T}_{\mathfrak{g}}(\Omega)$ and $\neg \mathcal{T}_{\mathfrak{g}}(\Omega) \supseteq \mathrm{C}_{\neg \mathcal{T}_{\mathfrak{g}}}^{\text {sup }}\left[\mathcal{S}_{\mathfrak{g}}\right]$ are true for the $\mathfrak{T}_{\mathfrak{g}}$-set $\mathcal{S}_{\mathfrak{g}} \subset \mathfrak{T}_{\mathfrak{g}}$ in question are clear from the context. To this end, the $\mathfrak{g}$-closure and the $\mathfrak{g}$-interior of a $\mathfrak{T}_{\mathfrak{g}}$-set $\mathcal{S}_{\mathfrak{g}} \subset \mathfrak{T}_{\mathfrak{g}}$ in a $\mathcal{T}_{\mathfrak{g}}$-space define themselves as

$$
\operatorname{int}_{\mathfrak{g}}\left(\mathcal{S}_{\mathfrak{g}}\right):=\bigcup_{\mathcal{O}_{\mathfrak{g}} \in \mathrm{C}_{\mathcal{T}_{\mathfrak{g}}}^{\text {sub }}\left[\mathcal{S}_{\mathfrak{g}}\right]} \mathcal{O}_{\mathfrak{g}} \text { and } \quad \operatorname{cl}_{\mathfrak{g}}\left(\mathcal{S}_{\mathfrak{g}}\right):=\bigcap_{\mathcal{K}_{\mathfrak{g}} \in \mathrm{C}_{\neg \mathcal{T}_{\mathfrak{g}}}^{\text {sup }}\left[\mathcal{S}_{\mathfrak{g}}\right]} \mathcal{K}_{\mathfrak{g}}
$$

We note in passing that, $\operatorname{cl}_{\mathfrak{g}}(\cdot) \neq \operatorname{cl}(\cdot)$ and $\operatorname{int}_{\mathfrak{g}}(\cdot) \neq \operatorname{int}(\cdot)$, because the resulting sets obtained from the intersection of all $\mathcal{T}_{\mathfrak{g}}$-closed supersets and the union of all $\mathcal{T}_{\mathfrak{g}}$-open subsets, respectively, relative to the $\mathfrak{g}$-topology $\mathcal{T}_{\mathfrak{g}}$ are not necessarily equal to those which would be obtained from the intersection of all $\mathcal{T}$-closed supersets and the union of all $\mathcal{T}$-open subsets relative to the topology $\mathcal{T}$ [23]. Throughout this work, by $\operatorname{cl}_{\mathfrak{g}} \circ \operatorname{int}_{\mathfrak{g}}(\cdot)$, $\operatorname{int}_{\mathfrak{g}} \circ \mathrm{cl}_{\mathfrak{g}}(\cdot)$, and $\operatorname{cl}_{\mathfrak{g}} \circ \operatorname{int}_{\mathfrak{g}} \circ \mathrm{cl}_{\mathfrak{g}}(\cdot)$, respectively, are meant $\operatorname{cl}_{\mathfrak{g}}\left(\operatorname{int}_{\mathfrak{g}}(\cdot)\right), \operatorname{int}_{\mathfrak{g}}\left(\mathrm{cl}_{\mathfrak{g}}(\cdot)\right)$, and $\operatorname{cl}_{\mathfrak{g}}\left(\operatorname{int}_{\mathfrak{g}}\left(\mathrm{cl}_{\mathfrak{g}}(\cdot)\right)\right)$; other composition operators are defined in a similar way. Also, the backslash $\mathfrak{T}_{\mathfrak{g}} \backslash \mathcal{S}_{\mathfrak{g}}$ refers to the set-theoretic relative complement of $\mathcal{S}_{\mathfrak{g}}$ in $\mathfrak{T}_{\mathfrak{g}}$. Finally, for convenience of notation, let $\mathcal{P}^{*}(\Omega)=\mathcal{P}(\Omega) \backslash\{\emptyset\}, \mathcal{T}_{\mathfrak{g}}^{*}=\mathcal{T}_{\mathfrak{g}} \backslash\{\emptyset\}$, and $\neg \mathcal{T}_{\mathfrak{g}}^{*}=\neg \mathcal{T}_{\mathfrak{g}} \backslash\{\emptyset\}$.

Definition 2.4 (g-Operation [39]). Let $\mathfrak{T}_{\mathfrak{g}}=\left(\Omega, \mathcal{T}_{\mathfrak{g}}\right)$ be a $\mathcal{T}_{\mathfrak{g}}$-space. Then, a mapping op $\mathfrak{g}: \mathcal{P}(\Omega) \longrightarrow$ $\mathcal{P}(\Omega)$ on $\mathcal{P}(\Omega)$ ranging in $\mathcal{P}(\Omega)$ is called a " $\mathfrak{g}$-operation" if and only if the following statements hold:

$$
\left(\forall \mathcal{S}_{\mathfrak{g}} \in \mathcal{P}^{*}(\Omega)\right)\left(\exists\left(\mathcal{O}_{\mathfrak{g}}, \mathcal{K}_{\mathfrak{g}}\right) \in \mathcal{T}_{\mathfrak{g}}^{*} \times \neg \mathcal{T}_{\mathfrak{g}}^{*}\right)\left[\left(\mathrm{op}_{\mathfrak{g}}(\emptyset)=\emptyset\right) \vee\left(\neg \mathrm{op}_{\mathfrak{g}}(\emptyset)=\emptyset\right) \vee\left(\mathcal{S}_{\mathfrak{g}} \subseteq \mathrm{op}_{\mathfrak{g}}\left(\mathcal{O}_{\mathfrak{g}}\right)\right) \vee\left(\mathcal{S}_{\mathfrak{g}} \supseteq \neg \mathrm{op}_{\mathfrak{g}}\left(\mathcal{K}_{\mathfrak{g}}\right)\right)\right]
$$

where $\neg \mathrm{op}_{\mathfrak{g}}: \mathcal{P}(\Omega) \longrightarrow \mathcal{P}(\Omega)$ is called the "complementary $\mathfrak{g}$-operation" on $\mathcal{P}(\Omega)$ ranging in $\mathcal{P}(\Omega)$ and, for all $\left(\mathcal{S}_{\mathfrak{g}}, \mathcal{U}_{\mathfrak{g}, \mu}, \mathcal{V}_{\mathfrak{g}, \nu}\right) \in \bigotimes_{\alpha \in I_{3}^{*}} \mathcal{P}^{*}(\Omega)$ such that $\mathcal{W}_{\mathfrak{g}}=\mathcal{U}_{\mathfrak{g}, \mu} \cup \mathcal{V}_{\mathfrak{g}, \nu}$ and $\left(\hat{\mathcal{W}}_{\mathfrak{g}}, \neg \hat{\mathcal{W}}_{\mathfrak{g}}\right)=$ $\left(\operatorname{op}_{\mathfrak{g}}\left(\mathcal{W}_{\mathfrak{g}}\right), \neg \mathrm{op}_{\mathfrak{g}}\left(\mathcal{W}_{\mathfrak{g}}\right)\right)$, the following axioms are satisfied: 
i. $\left(\mathcal{S}_{\mathfrak{g}} \subseteq \mathrm{op}_{\mathfrak{g}}\left(\mathcal{O}_{\mathfrak{g}}\right)\right) \vee\left(\mathcal{S}_{\mathfrak{g}} \supseteq \neg \mathrm{op}_{\mathfrak{g}}\left(\mathcal{K}_{\mathfrak{g}}\right)\right)$

ii. $\left(\operatorname{op}_{\mathfrak{g}}\left(\mathcal{S}_{\mathfrak{g}}\right) \subseteq \mathrm{op}_{\mathfrak{g}} \circ \mathrm{op}_{\mathfrak{g}}\left(\mathcal{O}_{\mathfrak{g}}\right)\right) \vee\left(\neg \mathrm{op}_{\mathfrak{g}}\left(\mathcal{S}_{\mathfrak{g}}\right) \supseteq \neg \mathrm{op}_{\mathfrak{g}} \circ \neg \mathrm{op}_{\mathfrak{g}}\left(\mathcal{K}_{\mathfrak{g}}\right)\right)$

iii. $\left(\hat{\mathcal{W}}_{\mathfrak{g}} \subseteq \bigcup_{\sigma=\mu, \nu} \operatorname{op}_{\mathfrak{g}}\left(\mathcal{O}_{\mathfrak{g}, \sigma}\right)\right) \bigvee\left(\neg \hat{\mathcal{W}}_{\mathfrak{g}} \supseteq \bigcup_{\sigma=\mu, \nu} \neg \mathrm{op}_{\mathfrak{g}}\left(\mathcal{K}_{\mathfrak{g}, \sigma}\right)\right)$

$i v .\left(\mathcal{U}_{\mathfrak{g}, \mu} \subseteq \mathcal{V}_{\mathfrak{g}, \nu} \longrightarrow \mathrm{op}_{\mathfrak{g}}\left(\mathcal{O}_{\mathfrak{g}, \mu}\right) \subseteq \mathrm{op}_{\mathfrak{g}}\left(\mathcal{O}_{\mathfrak{g}, \nu}\right)\right) \vee\left(\mathcal{U}_{\mathfrak{g}, \mu} \supseteq \mathcal{V}_{\mathfrak{g}, \nu} \longleftarrow \neg \mathrm{op}_{\mathfrak{g}}\left(\mathcal{K}_{\mathfrak{g}, \mu}\right) \supseteq \neg \mathrm{op}_{\mathfrak{g}}\left(\mathcal{K}_{\mathfrak{g}, \nu}\right)\right)$

for some $\left(\mathcal{O}_{\mathfrak{g}}, \mathcal{O}_{\mathfrak{g}, \mu}, \mathcal{O}_{\mathfrak{g}, \nu}\right) \in \bigotimes_{\alpha \in I_{3}^{*}} \mathcal{T}_{\mathfrak{g}}^{*}$ and $\left(\mathcal{K}_{\mathfrak{g}}, \mathcal{K}_{\mathfrak{g}, \mu}, \mathcal{K}_{\mathfrak{g}, \nu}\right) \in \bigotimes_{\alpha \in I_{3}^{*}} \neg \mathcal{T}_{\mathfrak{g}}^{*}$.

The formulation of Definition 2.5 is based on the axioms of the Cech closure operator [25] and the various axioms used by many mathematicians to define closure operators [36]. The class $\mathcal{L}_{\mathfrak{g}}[\Omega]$ stands for the class of all possible $\mathfrak{g}$-operators and their complementary $\mathfrak{g}$-operators in the $\mathcal{T}_{\mathfrak{g}}$-space $\mathfrak{T}_{\mathfrak{g}}$.

Definition $2.5\left(\operatorname{op}_{\mathfrak{g}}(\cdot)\right.$-Elements [39]). Let $\mathfrak{T}_{\mathfrak{g}}$ be a $\mathcal{T}_{\mathfrak{g}}$-space. The elements of the class $\mathcal{L}_{\mathfrak{g}}[\Omega]=$ $\mathcal{L}_{\mathfrak{g}}^{\omega}[\Omega] \times \mathcal{L}_{\mathfrak{g}}^{\kappa}[\Omega]$, where

$$
\mathcal{L}_{\mathfrak{g}}[\Omega]:=\left\{\mathbf{o p}_{\mathfrak{g}, \nu \mu}(\cdot)=\left(\mathrm{op}_{\mathfrak{g}, \nu}(\cdot), \neg \mathrm{op}_{\mathfrak{g}, \mu}(\cdot)\right):(\nu, \mu) \in I_{3}^{0} \times I_{3}^{0}\right\}
$$

in the $\mathcal{T}_{\mathfrak{g}}$-space $\mathfrak{T}_{\mathfrak{g}}$ are defined as:

$$
\begin{aligned}
\operatorname{op}_{\mathfrak{g}}(\cdot) & \in \mathcal{L}_{\mathfrak{g}}^{\omega}[\Omega]:=\left\{\operatorname{op}_{\mathfrak{g}, 0}(\cdot), \operatorname{op}_{\mathfrak{g}, 1}(\cdot), \operatorname{op}_{\mathfrak{g}, 2}(\cdot), \operatorname{op}_{\mathfrak{g}, 3}(\cdot)\right\} \\
& =\left\{\operatorname{int}_{\mathfrak{g}}(\cdot), \operatorname{cl}_{\mathfrak{g}} \circ \operatorname{int}_{\mathfrak{g}}(\cdot), \operatorname{int}_{\mathfrak{g}} \circ \mathrm{cl}_{\mathfrak{g}}(\cdot), \mathrm{cl}_{\mathfrak{g}} \circ \operatorname{int}_{\mathfrak{g}} \circ \mathrm{cl}_{\mathfrak{g}}(\cdot)\right\} \\
\neg \mathrm{op}_{\mathfrak{g}}(\cdot) & \in \mathcal{L}_{\mathfrak{g}}^{\kappa}[\Omega]:=\left\{\neg \operatorname{op}_{\mathfrak{g}, 0}(\cdot), \neg \mathrm{op}_{\mathfrak{g}, 1}(\cdot), \neg \mathrm{op}_{\mathfrak{g}, 2}(\cdot), \neg \mathrm{op}_{\mathfrak{g}, 3}(\cdot)\right\} \\
& =\left\{\operatorname{cl}_{\mathfrak{g}}(\cdot), \operatorname{int}_{\mathfrak{g}} \circ \operatorname{cl}_{\mathfrak{g}}(\cdot), \operatorname{cl}_{\mathfrak{g}} \circ \operatorname{int}_{\mathfrak{g}}(\cdot), \operatorname{int}_{\mathfrak{g}} \circ \operatorname{cl}_{\mathfrak{g}} \circ \operatorname{int}_{\mathfrak{g}}(\cdot)\right\}
\end{aligned}
$$

We remark in passing that, $\mathbf{o p}_{\mathfrak{g}, 11}(\cdot)=\neg \mathbf{o p}_{\mathfrak{g}, 22}(\cdot)$, and the use of $\mathbf{o p}_{\mathfrak{g}}(\cdot)=\left(\mathrm{op}_{\mathfrak{g}}(\cdot), \neg \mathrm{op}_{\mathfrak{g}}(\cdot)\right) \in$ $\mathcal{L}_{\mathfrak{g}}[\Omega]$ on a class of $\mathfrak{T}_{\mathfrak{g}}$-sets will construct a new class of $\mathfrak{g}-\mathfrak{T}_{\mathfrak{g}}$-sets, just as the use of $\mathcal{L}[\Omega]:=\left\{\mathbf{o p}_{\nu}(\cdot)=\right.$ $\left.\left(\operatorname{op}_{\nu}(\cdot), \neg \mathrm{op}_{\nu}(\cdot)\right): \quad \nu \in I_{3}^{0}\right\}$ on the class of $\mathfrak{T}$-sets have constructed the new class of $\mathfrak{g}$-T-sets. But since $\operatorname{cl}_{\mathfrak{g}}(\cdot) \neq \operatorname{cl}(\cdot)$ and $\operatorname{int}_{\mathfrak{g}}(\cdot) \neq \operatorname{int}(\cdot)$, in general, it follows that $\mathbf{o p}_{\mathfrak{g}}(\cdot) \neq \mathbf{o p}(\cdot)$ and, therefore, the new class of $\mathfrak{g}-\mathfrak{T}_{\mathfrak{g}}$-sets that will be obtained from the first construction will, in general, differ from the new class of $\mathfrak{g}$ - $\mathfrak{T}$-sets that had been obtained from the second construction.

Definition 2.6 $\left(\mathfrak{g}-\nu\right.$-T $\mathfrak{T}_{\mathfrak{g}}$-Set [39]). A $\mathfrak{T}_{\mathfrak{g}}$-set $\mathcal{S}_{\mathfrak{g}} \subset \mathfrak{T}_{\mathfrak{g}}$ in a $\mathcal{T}_{\mathfrak{g}}$-space is called a " $\mathfrak{g}$ - $\mathfrak{T}_{\mathfrak{g}}$-set" if and only if there exist a pair $\left(\mathcal{O}_{\mathfrak{g}}, \mathcal{K}_{\mathfrak{g}}\right) \in \mathcal{T}_{\mathfrak{g}} \times \neg \mathcal{T}_{\mathfrak{g}}$ of $\mathcal{T}_{\mathfrak{g}}$-open and $\mathcal{T}_{\mathfrak{g}}$-closed sets, and a $\mathfrak{g}$-operator op $\mathbf{o p}_{\mathfrak{g}}(\cdot) \in \mathcal{L}_{\mathfrak{g}}[\Omega]$ such that the following statement holds:

$$
(\exists \xi)\left[\left(\xi \in \mathcal{S}_{\mathfrak{g}}\right) \wedge\left(\left(\mathcal{S}_{\mathfrak{g}} \subseteq \mathrm{op}_{\mathfrak{g}}\left(\mathcal{O}_{\mathfrak{g}}\right)\right) \vee\left(\mathcal{S}_{\mathfrak{g}} \supseteq \neg \mathrm{op}_{\mathfrak{g}}\left(\mathcal{K}_{\mathfrak{g}}\right)\right)\right)\right]
$$

The $\mathfrak{g}$ - $\mathfrak{T}_{\mathfrak{g}}$-set $\mathcal{S}_{\mathfrak{g}} \subset \mathfrak{T}_{\mathfrak{g}}$ is said to be of category $\nu$ if and only if it belongs to the following class of $\mathfrak{g}-\nu$ - $\mathfrak{T}_{\mathfrak{g}}$-sets:

$$
\mathfrak{g}-\nu-\mathrm{S}\left[\mathfrak{T}_{\mathfrak{g}}\right]:=\left\{\mathcal{S}_{\mathfrak{g}} \subset \mathfrak{T}_{\mathfrak{g}}:\left(\exists \mathcal{O}_{\mathfrak{g}}, \mathcal{K}_{\mathfrak{g}}, \mathbf{o p}_{\mathfrak{g}, \nu}(\cdot)\right)\left[\left(\mathcal{S}_{\mathfrak{g}} \subseteq \mathrm{op}_{\mathfrak{g}, \nu}\left(\mathcal{O}_{\mathfrak{g}}\right)\right) \vee\left(\mathcal{S}_{\mathfrak{g}} \supseteq \neg \mathrm{op}_{\mathfrak{g}, \nu}\left(\mathcal{K}_{\mathfrak{g}}\right)\right)\right]\right\}
$$

It is called a $\mathfrak{g}-\nu-\mathfrak{T}_{\mathfrak{g}}$-open set if it satisfies the first property in $\mathfrak{g}-\nu-\mathrm{S}\left[\mathfrak{T}_{\mathfrak{g}}\right]$ and a $\mathfrak{g}-\nu-\mathfrak{T}_{\mathfrak{g}}$-closed set if it satisfies the second property in $\mathfrak{g}-\nu-\mathrm{S}\left[\mathfrak{T}_{\mathfrak{g}}\right]$. The classes of $\mathfrak{g}-\nu$ - T $\mathfrak{T}_{\mathfrak{g}}$-open and $\mathfrak{g}-\nu-\mathfrak{T}_{\mathfrak{g}}$-closed sets, respectively, are defined by

$$
\begin{aligned}
& \mathfrak{g}-\nu-\mathrm{O}\left[\mathfrak{T}_{\mathfrak{g}}\right]:=\left\{\mathcal{S}_{\mathfrak{g}} \subset \mathfrak{T}_{\mathfrak{g}}:\left(\exists \mathcal{O}_{\mathfrak{g}}, \mathbf{o p}_{\mathfrak{g}, \nu}(\cdot)\right)\left[\mathcal{S}_{\mathfrak{g}} \subseteq \mathrm{op}_{\mathfrak{g}, \nu}\left(\mathcal{O}_{\mathfrak{g}}\right)\right]\right\} \\
& \mathfrak{g}-\nu-\mathrm{K}\left[\mathfrak{T}_{\mathfrak{g}}\right]:=\left\{\mathcal{S}_{\mathfrak{g}} \subset \mathfrak{T}_{\mathfrak{g}}:\left(\exists \mathcal{K}_{\mathfrak{g}}, \mathbf{o p}_{\mathfrak{g}, \nu}(\cdot)\right)\left[\mathcal{S}_{\mathfrak{g}} \supseteq \neg \mathrm{op}_{\mathfrak{g}, \nu}\left(\mathcal{K}_{\mathfrak{g}}\right)\right]\right\}
\end{aligned}
$$

From the class $\mathfrak{g}-\nu-\mathrm{S}\left[\mathfrak{T}_{\mathfrak{g}}\right]$, consisting of the classes $\mathfrak{g}-\nu-\mathrm{O}\left[\mathfrak{T}_{\mathfrak{g}}\right]$ and $\mathfrak{g}-\nu-\mathrm{K}\left[\mathfrak{T}_{\mathfrak{g}}\right]$, respectively, of $\mathfrak{g}-\nu-\mathfrak{T}_{\mathfrak{g}}$ open and $\mathfrak{g}-\nu-\mathfrak{T}_{\mathfrak{g}}$-closed sets of category $\nu$, where $\nu \in I_{3}^{0}$, there results in the following definition. 
Definition $2.7\left(\mathfrak{g}-\mathfrak{T}_{\mathfrak{g}}\right.$-Set $\left.[39]\right)$. Let $\mathfrak{T}_{\mathfrak{g}}$ be a $\mathcal{T}_{\mathfrak{g}}$-space. If, for each $\nu \in I_{3}^{0}, \mathfrak{g}-\nu-\mathrm{O}\left[\mathfrak{T}_{\mathfrak{g}}\right]$ and $\mathfrak{g}-\nu-\mathrm{K}\left[\mathfrak{T}_{\mathfrak{g}}\right]$, respectively, denote the classes of $\mathfrak{g}-\nu-\mathfrak{T}_{\mathfrak{g}}$-open and $\mathfrak{g}-\nu$ - $\mathfrak{T}_{\mathfrak{g}}$-closed sets of category $\nu$ then,

$$
\begin{aligned}
\mathfrak{g}-\mathrm{S}\left[\mathfrak{T}_{\mathfrak{g}}\right]=\bigcup_{\nu \in I_{3}^{0}} \mathfrak{g}-\nu-\mathrm{S}\left[\mathfrak{T}_{\mathfrak{g}}\right] & =\bigcup_{\nu \in I_{3}^{0}}\left(\mathfrak{g}-\nu-\mathrm{O}\left[\mathfrak{T}_{\mathfrak{g}}\right] \cup \mathfrak{g}-\nu-\mathrm{K}\left[\mathfrak{T}_{\mathfrak{g}}\right]\right) \\
& =\left(\bigcup_{\nu \in I_{3}^{0}} \mathfrak{g}-\nu-\mathrm{O}\left[\mathfrak{T}_{\mathfrak{g}}\right]\right) \cup\left(\bigcup_{\nu \in I_{3}^{0}} \mathfrak{g}-\nu-\mathrm{K}\left[\mathfrak{T}_{\mathfrak{g}}\right]\right) \\
& =\mathfrak{g}-\mathrm{O}\left[\mathfrak{T}_{\mathfrak{g}}\right] \cup \mathfrak{g}-\mathrm{K}\left[\mathfrak{T}_{\mathfrak{g}}\right]
\end{aligned}
$$

In the sequel, it is interesting to view the concepts of open, semi-open, pre-open, semi-pre-open sets as $\mathfrak{g}$-T-T-open sets of categories $0,1,2$, and 3; likewise, to view the concepts of closed, semi-closed, pre-closed, semi-pre-closed sets as $\mathfrak{g}$-T-closed sets of categories $0,1,2$, and 3 . These can be realised by omitting the subscript " $\mathfrak{g}$ " in all symbols of the above definitions.

Definition $2.8(\mathfrak{g}$ - $\nu$-T $-S e t[39])$. A $\mathfrak{T}$-set $\mathcal{S} \subset \mathfrak{T}$ in a $\mathcal{T}$-space is called a " $\mathfrak{g}$-T-set" if and only if there exists a pair $(\mathcal{O}, \mathcal{K}) \in \mathcal{T} \times \neg \mathcal{T}$ of $\mathcal{T}$-open and $\mathcal{T}$-closed sets, and an operator op $(\cdot) \in \mathcal{L}[\Omega]$ such that the following statement holds:

$$
(\exists \xi)[(\xi \in \mathcal{S}) \wedge((\mathcal{S} \subseteq \text { op }(\mathcal{O})) \vee(\mathcal{S} \supseteq \neg \text { op }(\mathcal{K})))]
$$

The $\mathfrak{g}$ - $\mathfrak{T}$-set $\mathcal{S} \subset \mathfrak{T}$ is said to be of category $\nu$ if and only if it belongs to the following class of $\mathfrak{g}-\nu$ - $\mathcal{T}$-sets:

$$
\mathfrak{g}-\nu-\mathrm{S}[\mathfrak{T}]:=\left\{\mathcal{S} \subset \mathfrak{T}:\left(\exists \mathcal{O}, \mathcal{K}, \operatorname{op}_{\nu}(\cdot)\right)\left[\left(\mathcal{S} \subseteq \operatorname{op}_{\nu}(\mathcal{O})\right) \vee\left(\mathcal{S} \supseteq \neg \operatorname{op}_{\nu}(\mathcal{K})\right)\right]\right\}
$$

It is called a $\mathfrak{g}-\nu$-T-open set if it satisfies the first property in $\mathfrak{g}-\nu-\mathrm{S}[\mathfrak{T}]$ and a $\mathfrak{g}-\nu$-T-closed set if it satisfies the second property in $\mathfrak{g}-\nu-\mathrm{S}[\mathfrak{T}]$. The classes of $\mathfrak{g}-\nu$ - $\mathfrak{T}$-open and $\mathfrak{g}-\nu$ - $\mathfrak{T}$-closed sets, respectively, are defined by

$$
\begin{aligned}
& \mathfrak{g}-\nu-\mathrm{O}[\mathfrak{T}]:=\left\{\mathcal{S} \subset \mathfrak{T}:\left(\exists \mathcal{O}, \mathbf{o p}_{\nu}(\cdot)\right)\left[\mathcal{S} \subseteq \mathrm{op}_{\nu}(\mathcal{O})\right]\right\} \\
& \mathfrak{g}-\nu-\mathrm{K}[\mathfrak{T}]:=\left\{\mathcal{S} \subset \mathfrak{T}:\left(\exists \mathcal{K}, \mathbf{o p}_{\nu}(\cdot)\right)\left[\mathcal{S} \supseteq \neg \operatorname{op}_{\nu}(\mathcal{K})\right]\right\}
\end{aligned}
$$

As in the previous definitions, from the class $\mathfrak{g}-\nu-\mathrm{S}[\mathfrak{T}]$, consisting of the classes $\mathfrak{g}-\nu-\mathrm{O}[\mathfrak{T}]$ and $\mathfrak{g}-\nu$-K $[\mathfrak{T}]$, respectively, of $\mathfrak{g}-\nu$-T-Topen and $\mathfrak{g}-\nu$-T-closed sets of category $\nu$, where $\nu \in I_{3}^{0}$, there results in the following definition.

Definition 2.9 (Class: $\mathfrak{g}$ - $\mathfrak{T}_{\mathfrak{g}}$-Sets [39]). Let $\mathfrak{T}$ be a $\mathcal{T}$-space. If, for each $\nu \in I_{3}^{0}, \mathfrak{g}-\nu$-O $[\mathfrak{T}]$ and $\mathfrak{g}-\nu$-K $[\mathfrak{T}]$, respectively, denote the classes of $\mathfrak{g}-\nu$ - $\mathfrak{T}$-open and $\mathfrak{g}-\nu$ - $\mathfrak{T}$-closed sets of category $\nu$ then,

$$
\begin{aligned}
\mathfrak{g}-\mathrm{S}[\mathfrak{T}]=\bigcup_{\nu \in I_{3}^{0}} \mathfrak{g}-\nu-\mathrm{S}[\mathfrak{T}] & =\bigcup_{\nu \in I_{3}^{0}}(\mathfrak{g}-\nu-\mathrm{O}[\mathfrak{T}] \cup \mathfrak{g}-\nu-\mathrm{K}[\mathfrak{T}]) \\
& =\left(\bigcup_{\nu \in I_{3}^{0}} \mathfrak{g}-\nu-\mathrm{O}[\mathfrak{T}]\right) \cup\left(\bigcup_{\nu \in I_{3}^{0}} \mathfrak{g}-\nu-\mathrm{K}[\mathfrak{T}]\right) \\
& =\mathfrak{g}-\mathrm{O}[\mathfrak{T}] \cup \mathfrak{g}-\mathrm{K}[\mathfrak{T}]
\end{aligned}
$$

The classes of $\mathfrak{T}_{\mathfrak{g}}$-open and $\mathfrak{T}_{\mathfrak{g}}$-closed sets in a $\mathcal{T}_{\mathfrak{g}}$-space $\mathfrak{T}_{\mathfrak{g}}$ as well as the classes of $\mathfrak{T}$-open and $\mathfrak{T}$-closed sets in a $\mathcal{T}$-space $\mathfrak{T}$ are defined as thus:

Definition 2.10 (Families: $\mathfrak{g}$ - $\mathfrak{T}_{\mathfrak{g}}$-Open Sets, $\mathfrak{g}$ - $\mathfrak{T}_{\mathfrak{g}}$-Closed Sets $\left.[39]\right)$. Let $\mathfrak{T}_{\mathfrak{g}}=\left(\Omega, \mathcal{T}_{\mathfrak{g}}\right)$ be a $\mathcal{T}_{\mathfrak{g}}$-space and let $\mathfrak{T}=(\Omega, \mathcal{T})$ be a $\mathcal{T}$-space.

i. The classes $\mathrm{O}\left[\mathfrak{T}_{\mathfrak{g}}\right]$ and $\mathrm{K}\left[\mathfrak{T}_{\mathfrak{g}}\right]$ denote the families of $\mathfrak{T}_{\mathfrak{g}}$-open and $\mathfrak{T}_{\mathfrak{g}}$-closed sets, respectively, in $\mathfrak{T}_{\mathfrak{g}}$, with $\mathrm{S}\left[\mathfrak{T}_{\mathfrak{g}}\right]=\mathrm{O}\left[\mathfrak{T}_{\mathfrak{g}}\right] \cup \mathrm{K}\left[\mathfrak{T}_{\mathfrak{g}}\right]$.

ii. The classes $\mathrm{O}[\mathfrak{T}]$ and $\mathrm{K}[\mathfrak{T}]$ denote the families of $\mathfrak{T}$-open and $\mathfrak{T}$-closed sets, respectively, in $\mathfrak{T}$, with $\mathrm{S}[\mathfrak{T}]=\mathrm{O}[\mathfrak{T}] \cup \mathrm{K}[\mathfrak{T}]$.

In the following sections, the main results of the theory of $\mathfrak{g}-\mathfrak{T}_{\mathfrak{g}}$-sets are presented. 


\section{Main Results}

Theorem 3.1. Let $\mathrm{cl}_{\mathfrak{g}}: \mathcal{P}(\Omega) \longrightarrow \mathcal{P}(\Omega)$ and $\operatorname{int}_{\mathfrak{g}}: \mathcal{P}(\Omega) \longrightarrow \mathcal{P}(\Omega)$, respectively, be $\mathfrak{g}$-closure and $\mathfrak{g}$-interior operators in the $\mathcal{T}_{\mathfrak{g}}$-space $\mathfrak{T}_{\mathfrak{g}}$. Then,

$i . \mathrm{cl}_{\mathfrak{g}}(\cdot)$ and $\operatorname{int}_{\mathfrak{g}}(\cdot)$ are enhancing and contracting, respectively.

ii. $\operatorname{cl}_{\mathfrak{g}}(\cdot)$ and $\operatorname{int}_{\mathfrak{g}}(\cdot)$ are idempotent.

iii. $\operatorname{cl}_{\mathfrak{g}}(\cdot)$ and $\operatorname{int}_{\mathfrak{g}}(\cdot)$ are monotone.

\section{Proof.}

$i$. Since the following logical statement

$$
\mathcal{S}_{\mathfrak{g}} \subset \mathfrak{T}_{\mathfrak{g}}:(\forall \xi)\left[\left(\xi \in \mathrm{cl}_{\mathfrak{g}}\left(\mathcal{S}_{\mathfrak{g}}\right) \longleftarrow \xi \in \mathcal{S}_{\mathfrak{g}}\right) \vee\left(\xi \in \operatorname{int}_{\mathfrak{g}}\left(\mathcal{S}_{\mathfrak{g}}\right) \longrightarrow \xi \in \mathcal{S}_{\mathfrak{g}}\right)\right]
$$

holds, it follows that $\mathcal{S}_{\mathfrak{g}} \subseteq \operatorname{cl}_{\mathfrak{g}}\left(\mathcal{S}_{\mathfrak{g}}\right)$ or $\mathcal{S}_{\mathfrak{g}} \supseteq \operatorname{int}_{\mathfrak{g}}\left(\mathcal{S}_{\mathfrak{g}}\right)$.

ii. If $\mathcal{S}_{\mathfrak{g}}$ is open, then $\mathcal{S}_{\mathfrak{g}}=\operatorname{int}_{\mathfrak{g}}\left(\mathcal{S}_{\mathfrak{g}}\right)$; if it is closed, $\mathcal{S}_{\mathfrak{g}}=\operatorname{cl}_{\mathfrak{g}}\left(\mathcal{S}_{\mathfrak{g}}\right)$. Consequently, the substitutions $\mathcal{S}_{\mathfrak{g}} \longmapsto \operatorname{int}_{\mathfrak{g}}\left(\mathcal{S}_{\mathfrak{g}}\right)$ and $\mathcal{S}_{\mathfrak{g}} \longmapsto \operatorname{cl}_{\mathfrak{g}}\left(\mathcal{S}_{\mathfrak{g}}\right)$, respectively, give $\operatorname{int}_{\mathfrak{g}}\left(\mathcal{S}_{\mathfrak{g}}\right)=\operatorname{int}_{\mathfrak{g}} \circ \operatorname{int}_{\mathfrak{g}}\left(\mathcal{S}_{\mathfrak{g}}\right)$ and $\operatorname{cl}_{\mathfrak{g}}\left(\mathcal{S}_{\mathfrak{g}}\right)=$ $\operatorname{cl}_{\mathfrak{g}} \circ \mathrm{cl}_{\mathfrak{g}}\left(\mathcal{S}_{\mathfrak{g}}\right)$.

iii. Let $\mathcal{R}_{\mathfrak{g}}, \mathcal{S}_{\mathfrak{g}} \subset \mathfrak{T}_{\mathfrak{g}}$ such that $\mathcal{R}_{\mathfrak{g}} \subseteq \mathcal{S}_{\mathfrak{g}}$. Then, $\mathcal{R}_{\mathfrak{g}} \subseteq \mathrm{cl}_{\mathfrak{g}}\left(\mathcal{R}_{\mathfrak{g}}\right), \mathcal{R}_{\mathfrak{g}} \supseteq \operatorname{int}_{\mathfrak{g}}\left(\mathcal{R}_{\mathfrak{g}}\right), \mathcal{S}_{\mathfrak{g}} \subseteq \mathrm{cl}_{\mathfrak{g}}\left(\mathcal{S}_{\mathfrak{g}}\right)$, and $\mathcal{S}_{\mathfrak{g}} \supseteq \operatorname{int}_{\mathfrak{g}}\left(\mathcal{S}_{\mathfrak{g}}\right)$ by $i$. Consequently, $\operatorname{int}_{\mathfrak{g}}\left(\mathcal{R}_{\mathfrak{g}}\right) \subseteq \operatorname{int}_{\mathfrak{g}}\left(\mathcal{S}_{\mathfrak{g}}\right)$ and $\operatorname{cl}_{\mathfrak{g}}\left(\mathcal{R}_{\mathfrak{g}}\right) \subseteq \operatorname{cl}_{\mathfrak{g}}\left(\mathcal{S}_{\mathfrak{g}}\right)$.

Lemma 3.2. Let $\mathcal{S}_{\mathfrak{g}} \subset \mathfrak{T}_{\mathfrak{g}}$ be a $\mathfrak{T}_{\mathfrak{g}}$-set of a $\mathcal{T}_{\mathfrak{g}}$-space. Then,

i. $\left(\mathcal{S}_{\mathfrak{g}}=\emptyset\right) \wedge\left(\Omega \in \mathcal{T}_{\mathfrak{g}}\right) \Rightarrow\left(\operatorname{int}_{\mathfrak{g}}\left(\mathcal{S}_{\mathfrak{g}}\right)=\emptyset\right) \wedge\left(\mathrm{cl}_{\mathfrak{g}}(\emptyset)=\emptyset\right)$

ii. $\left(\mathcal{S}_{\mathfrak{g}}=\emptyset\right) \wedge\left(\Omega \notin \mathcal{T}_{\mathfrak{g}}\right) \Rightarrow\left(\operatorname{int}_{\mathfrak{g}}\left(\mathcal{S}_{\mathfrak{g}}\right)=\emptyset\right) \wedge\left(\mathrm{cl}_{\mathfrak{g}}(\emptyset) \neq \emptyset\right)$

\section{Proof.}

i. If $\mathcal{S}_{\mathfrak{g}}=\emptyset$ and $\Omega \in \mathcal{T}_{\mathfrak{g}}$, then $\left(\emptyset \in \mathrm{C}_{\mathcal{T}_{\mathfrak{g}}}^{\text {sub }}[\emptyset]\right) \wedge\left(\emptyset \in \mathrm{C}_{\mathcal{T}_{\mathfrak{g}}}^{\text {sup }}[\emptyset]\right)$. Consequently, $\operatorname{int}_{\mathfrak{g}}(\emptyset)=\emptyset$ and $\operatorname{cl}_{\mathfrak{g}}(\emptyset)=\emptyset$.

ii. If $\mathcal{S}_{\mathfrak{g}}=\emptyset$ and $\Omega \notin \mathcal{T}_{\mathfrak{g}}$, then $\left(\emptyset \in \mathrm{C}_{\mathcal{T}_{\mathfrak{g}}}^{\text {sub }}[\emptyset]\right) \wedge\left(\emptyset \notin \mathrm{C}_{\mathcal{T}_{\mathfrak{g}}}^{\text {sup }}[\emptyset]\right)$. Consequently, $\operatorname{int}_{\mathfrak{g}}(\emptyset)=\emptyset$ and $\operatorname{int}_{\mathfrak{g}}(\emptyset) \neq \emptyset$.

According to Sarsak [40] and Noiri [41], the $\mathcal{T}_{\mathfrak{g}}$-space $\mathfrak{T}_{\mathfrak{g}}$ may be called a $\mu$-space when $\mathrm{cl}_{\mathfrak{g}}(\emptyset)=\emptyset$.

Theorem 3.3. If $\mathcal{S}_{\mathfrak{g}, 1}, \mathcal{S}_{\mathfrak{g}, 2}, \ldots, \mathcal{S}_{\mathfrak{g}, n} \subset \mathfrak{T}_{\mathfrak{g}}$ are $n \geq 1 \mathfrak{T}_{\mathfrak{g}}$-sets of a $\mathcal{T}_{\mathfrak{g}}$-space, then,

i. $\operatorname{cl}_{\mathfrak{g}}\left(\bigcup_{\nu \in I_{n}^{*}} \mathcal{S}_{\mathfrak{g}, \nu}\right)=\bigcup_{\nu \in I_{n}^{*}} \operatorname{cl}_{\mathfrak{g}}\left(\mathcal{S}_{\mathfrak{g}, \nu}\right)$

ii. $\operatorname{int}_{\mathfrak{g}}\left(\bigcap_{\nu \in I_{n}^{*}} \mathcal{S}_{\mathfrak{g}, \nu}\right)=\bigcap_{\nu \in I_{n}^{*}} \operatorname{int}_{\mathfrak{g}}\left(\mathcal{S}_{\mathfrak{g}, \nu}\right)$

Proof. Expressed in set-builder notation, the $\mathfrak{g}$-closure and the $\mathfrak{g}$-interior of a $\mathfrak{T}_{\mathfrak{g}}$-set $\mathcal{S}_{\mathfrak{g}} \subset \mathfrak{T}_{\mathfrak{g}}$ in a $\mathcal{T}_{\mathfrak{g}}$-space can also be defined as thus:

$$
\begin{aligned}
\operatorname{cl}_{\mathfrak{g}}\left(\mathcal{S}_{\mathfrak{g}}\right) & :=\left\{\xi \in \mathfrak{T}_{\mathfrak{g}}:\left(\mathcal{S}_{\mathfrak{g}} \cap \operatorname{cl}\left(\mathcal{O}_{\mathfrak{g}}\right) \neq \emptyset\right) \wedge\left(\xi \in \mathcal{O}_{\mathfrak{g}} \in \mathcal{T}_{\mathfrak{g}}\right)\right\} \\
\operatorname{int}_{\mathfrak{g}}\left(\mathcal{S}_{\mathfrak{g}}\right) & :=\left\{\xi \in \mathfrak{T}_{\mathfrak{g}}:\left(\mathcal{S}_{\mathfrak{g}} \cap \operatorname{int}\left(\mathcal{O}_{\mathfrak{g}}\right)=\operatorname{int}\left(\mathcal{O}_{\mathfrak{g}}\right)\right) \wedge\left(\xi \in \mathcal{O}_{\mathfrak{g}} \in \mathcal{T}_{\mathfrak{g}}\right)\right\}
\end{aligned}
$$


respectively, from which it is easily seen that,

$$
\begin{aligned}
\mathrm{cl}_{\mathfrak{g}}\left(\bigcup_{\nu \in I_{n}^{*}} \mathcal{S}_{\mathfrak{g}, \nu}\right) & =\bigcup_{\nu \in I_{n}^{*}}\left\{\xi \in \mathfrak{T}_{\mathfrak{g}}:\left(\mathcal{S}_{\mathfrak{g}, \nu} \cap \operatorname{cl}\left(\mathcal{O}_{\mathfrak{g}}\right) \neq \emptyset\right) \wedge\left(\xi \in \mathcal{O}_{\mathfrak{g}} \in \mathcal{T}_{\mathfrak{g}}\right)\right\} \\
& =\left\{\xi \in \mathfrak{T}_{\mathfrak{g}}:\left(\left(\bigcup_{\nu \in I_{n}^{*}} \mathcal{S}_{\mathfrak{g}, \nu}\right) \cap \operatorname{cl}\left(\mathcal{O}_{\mathfrak{g}}\right) \neq \emptyset\right) \wedge\left(\xi \in \mathcal{O}_{\mathfrak{g}} \in \mathcal{T}_{\mathfrak{g}}\right)\right\} \\
& =\left\{\xi \in \mathfrak{T}_{\mathfrak{g}}:\left(\bigcup_{\nu \in I_{n}^{*}}\left(\mathcal{S}_{\mathfrak{g}, \nu} \cap \operatorname{cl}\left(\mathcal{O}_{\mathfrak{g}}\right)\right) \neq \emptyset\right) \wedge\left(\xi \in \mathcal{O}_{\mathfrak{g}} \in \mathcal{T}_{\mathfrak{g}}\right)\right\} \\
& =\left\{\xi \in \mathfrak{T}_{\mathfrak{g}}: \bigvee_{\nu \in I_{n}^{*}}\left(\left(\mathcal{S}_{\mathfrak{g}, \nu} \cap \operatorname{cl}\left(\mathcal{O}_{\mathfrak{g}}\right) \neq \emptyset\right) \wedge\left(\xi \in \mathcal{O}_{\mathfrak{g}} \in \mathcal{T}_{\mathfrak{g}}\right)\right)\right\} \\
& =\bigcup_{\nu \in I_{n}^{*}} \operatorname{cl}_{\mathfrak{g}}\left(\mathcal{S}_{\mathfrak{g}, \nu}\right)
\end{aligned}
$$

Likewise, it is also easily seen that,

$$
\begin{aligned}
\operatorname{int}_{\mathfrak{g}}\left(\bigcap_{\nu \in I_{n}^{*}} \mathcal{S}_{\mathfrak{g}, \nu}\right) & =\bigcap_{\nu \in I_{n}^{*}}\left\{\xi \in \mathfrak{T}_{\mathfrak{g}}:\left(\mathcal{S}_{\mathfrak{g}, \nu} \cap \operatorname{int}\left(\mathcal{O}_{\mathfrak{g}}\right)=\operatorname{int}\left(\mathcal{O}_{\mathfrak{g}}\right)\right) \wedge\left(\xi \in \mathcal{O}_{\mathfrak{g}} \in \mathcal{T}_{\mathfrak{g}}\right)\right\} \\
& =\left\{\xi \in \mathfrak{T}_{\mathfrak{g}}:\left(\left(\bigcap_{\nu \in I_{n}^{*}} \mathcal{S}_{\mathfrak{g}, \nu}\right) \cap \operatorname{int}\left(\mathcal{O}_{\mathfrak{g}}\right)=\operatorname{int}\left(\mathcal{O}_{\mathfrak{g}}\right)\right) \wedge\left(\xi \in \mathcal{O}_{\mathfrak{g}} \in \mathcal{T}_{\mathfrak{g}}\right)\right\} \\
& =\left\{\xi \in \mathfrak{T}_{\mathfrak{g}}:\left(\bigcap_{\nu \in I_{n}^{*}}\left(\mathcal{S}_{\mathfrak{g}, \nu} \cap \operatorname{int}\left(\mathcal{O}_{\mathfrak{g}}\right)\right)=\operatorname{int}\left(\mathcal{O}_{\mathfrak{g}}\right)\right) \wedge\left(\xi \in \mathcal{O}_{\mathfrak{g}} \in \mathcal{T}_{\mathfrak{g}}\right)\right\} \\
& =\left\{\xi \in \mathfrak{T}_{\mathfrak{g}}: \bigwedge_{\nu \in I_{n}^{*}}\left(\left(\mathcal{S}_{\mathfrak{g}, \nu} \cap \operatorname{int}\left(\mathcal{O}_{\mathfrak{g}}\right)=\operatorname{int}\left(\mathcal{O}_{\mathfrak{g}}\right)\right) \wedge\left(\xi \in \mathcal{O}_{\mathfrak{g}} \in \mathcal{T}_{\mathfrak{g}}\right)\right)\right\} \\
& =\bigcap_{\nu \in I_{n}^{*}} \operatorname{int}_{\mathfrak{g}}\left(\mathcal{S}_{\mathfrak{g}, \nu}\right)
\end{aligned}
$$

Clearly, $\mathcal{S}_{\mathfrak{g}, \mu} \subseteq \bigcup_{\nu \in I_{n}^{*}} \mathcal{S}_{\mathfrak{g}, \nu}$ and $\mathcal{S}_{\mathfrak{g}, \mu} \supseteq \bigcap_{\nu \in I_{n}^{*}} \mathcal{S}_{\mathfrak{g}, \nu}$ hold true for any $\mu \in I_{n}^{*}$. The following corollary, then, is an immediate consequence of the above theorem.

Corollary 3.4. If $\mathcal{S}_{\mathfrak{g}, 1}, \mathcal{S}_{\mathfrak{g}, 2}, \ldots, \mathcal{S}_{\mathfrak{g}, n} \subset \mathfrak{T}_{\mathfrak{g}}$ are $n \geq 1 \mathfrak{T}_{\mathfrak{g}}$-sets of a $\mathcal{T}_{\mathfrak{g}}$-space, then,

i. $\operatorname{cl}_{\mathfrak{g}}\left(\bigcap_{\nu \in I_{n}^{*}} \mathcal{S}_{\mathfrak{g}, \nu}\right) \subseteq \bigcap_{\nu \in I_{n}^{*}} \operatorname{cl}_{\mathfrak{g}}\left(\mathcal{S}_{\mathfrak{g}, \nu}\right)$

ii. $\operatorname{int}_{\mathfrak{g}}\left(\bigcup_{\nu \in I_{n}^{*}} \mathcal{S}_{\mathfrak{g}, \nu}\right) \supseteq \bigcup_{\nu \in I_{n}^{*}} \operatorname{int}_{\mathfrak{g}}\left(\mathcal{S}_{\mathfrak{g}, \nu}\right)$

Proposition 3.5. For any $\mathfrak{T}_{\mathfrak{g}}$-set $\mathcal{S}_{\mathfrak{g}} \subset \mathfrak{T}_{\mathfrak{g}}$ in a $\mathcal{T}_{\mathfrak{g}}$-space $\mathfrak{T}_{\mathfrak{g}}$, the following statement holds:

$$
\mathfrak{T}_{\mathfrak{g}} \backslash\left(\operatorname{int}_{\mathfrak{g}}\left(\mathcal{S}_{\mathfrak{g}}\right) \cup \operatorname{cl}_{\mathfrak{g}}\left(\mathfrak{T}_{\mathfrak{g}} \backslash \mathcal{S}_{\mathfrak{g}}\right)\right)=\emptyset
$$

Proof. Let $\xi \in \mathrm{cl}_{\mathfrak{g}}\left(\mathfrak{T}_{\mathfrak{g}} \backslash \mathcal{S}_{\mathfrak{g}}\right)$. Then, $\xi \in \mathfrak{T}_{\mathfrak{g}} \backslash \mathcal{S}_{\mathfrak{g}}$ since, $\mathfrak{T}_{\mathfrak{g}} \backslash \mathcal{S}_{\mathfrak{g}} \subseteq \mathrm{cl}_{\mathfrak{g}}\left(\mathfrak{T}_{\mathfrak{g}} \backslash \mathcal{S}_{\mathfrak{g}}\right)$. But, $\mathfrak{T}_{\mathfrak{g}} \backslash \mathcal{S}_{\mathfrak{g}} \subseteq \mathfrak{T}_{\mathfrak{g}} \backslash$ $\operatorname{int}_{\mathfrak{g}}\left(\mathcal{S}_{\mathfrak{g}}\right) \subseteq \mathrm{cl}_{\mathfrak{g}}\left(\mathfrak{T}_{\mathfrak{g}} \backslash \mathcal{S}_{\mathfrak{g}}\right)$ and, consequently, $\xi \in \mathfrak{T}_{\mathfrak{g}} \backslash \operatorname{int}_{\mathfrak{g}}\left(\mathcal{S}_{\mathfrak{g}}\right)$. Hence, there follows that, cl $\mathrm{g}_{\mathfrak{g}}\left(\mathfrak{T}_{\mathfrak{g}} \backslash \mathcal{S}_{\mathfrak{g}}\right) \subseteq$ $\mathfrak{T}_{\mathfrak{g}} \backslash \operatorname{int}_{\mathfrak{g}}\left(\mathcal{S}_{\mathfrak{g}}\right)$. Conversely, let $\xi \in \mathfrak{T}_{\mathfrak{g}} \backslash \operatorname{int}_{\mathfrak{g}}\left(\mathcal{S}_{\mathfrak{g}}\right)$. Then, $\xi \in \operatorname{cl}_{\mathfrak{g}}\left(\mathfrak{T}_{\mathfrak{g}} \backslash \operatorname{int}_{\mathfrak{g}}\left(\mathcal{S}_{\mathfrak{g}}\right)\right)$, since $\mathfrak{T}_{\mathfrak{g}} \backslash \operatorname{int}_{\mathfrak{g}}\left(\mathcal{S}_{\mathfrak{g}}\right) \subseteq$ $\operatorname{cl}_{\mathfrak{g}}\left(\mathfrak{T}_{\mathfrak{g}} \backslash \operatorname{int}_{\mathfrak{g}}\left(\mathcal{S}_{\mathfrak{g}}\right)\right)$. But, since $\mathfrak{T}_{\mathfrak{g}} \backslash \operatorname{int}_{\mathfrak{g}}\left(\mathcal{S}_{\mathfrak{g}}\right) \subseteq \operatorname{cl}_{\mathfrak{g}}\left(\mathfrak{T}_{\mathfrak{g}} \backslash \mathcal{S}_{\mathfrak{g}}\right)$ and $\operatorname{cl}_{\mathfrak{g}}\left(\mathfrak{T}_{\mathfrak{g}} \backslash \mathcal{S}_{\mathfrak{g}}\right) \subseteq \operatorname{cl}_{\mathfrak{g}}\left(\mathfrak{T}_{\mathfrak{g}} \backslash \operatorname{int}_{\mathfrak{g}}\left(\mathcal{S}_{\mathfrak{g}}\right)\right)$, and, consequently, $\xi \in \mathfrak{T}_{\mathfrak{g}} \backslash \operatorname{int}_{\mathfrak{g}}\left(\mathcal{S}_{\mathfrak{g}}\right)$. Hence, $\mathfrak{T}_{\mathfrak{g}} \backslash \operatorname{int}_{\mathfrak{g}}\left(\mathcal{S}_{\mathfrak{g}}\right) \subseteq \operatorname{cl}_{\mathfrak{g}}\left(\mathfrak{T}_{\mathfrak{g}} \backslash \mathcal{S}_{\mathfrak{g}}\right)$. Since $\operatorname{cl}_{\mathfrak{g}}\left(\mathfrak{T}_{\mathfrak{g}} \backslash \mathcal{S}_{\mathfrak{g}}\right)=\mathfrak{T}_{\mathfrak{g}} \backslash \operatorname{int}_{\mathfrak{g}}\left(\mathcal{S}_{\mathfrak{g}}\right)$ is equivalent to

$$
\left(\operatorname{cl}_{\mathfrak{g}}\left(\mathfrak{T}_{\mathfrak{g}} \backslash \mathcal{S}_{\mathfrak{g}}\right) \subseteq \mathfrak{T}_{\mathfrak{g}} \backslash \operatorname{int}_{\mathfrak{g}}\left(\mathcal{S}_{\mathfrak{g}}\right)\right) \wedge\left(\operatorname{cl}_{\mathfrak{g}}\left(\mathfrak{T}_{\mathfrak{g}} \backslash \mathcal{S}_{\mathfrak{g}}\right) \supseteq \mathfrak{T}_{\mathfrak{g}} \backslash \operatorname{int}_{\mathfrak{g}}\left(\mathcal{S}_{\mathfrak{g}}\right)\right)
$$

the proof of the proposition at once follows.

Proposition 3.6. Let $\mathrm{cl}_{\mathfrak{g}}: \mathcal{P}(\Omega) \longrightarrow \mathcal{P}(\Omega)$ and $\operatorname{int}_{\mathfrak{g}}: \mathcal{P}(\Omega) \longrightarrow \mathcal{P}(\Omega)$, respectively, be $\mathfrak{g}$-closure and $\mathfrak{g}$-interior operators in a $\mathcal{T}_{\mathfrak{g}}$-space $\mathfrak{T}_{\mathfrak{g}}$. If $\mathcal{S}_{\mathfrak{g}, 1}, \mathcal{S}_{\mathfrak{g}, 2}, \ldots, \mathcal{S}_{\mathfrak{g}, n} \subset \mathfrak{T}_{\mathfrak{g}}$ are $n \geq 1 \mathfrak{T}_{\mathfrak{g}}$-sets of the $\mathcal{T}_{\mathfrak{g}}$-space $\mathfrak{T}_{\mathfrak{g}}$, then,
i. $\operatorname{cl}_{\mathfrak{g}} \circ \operatorname{int}_{\mathfrak{g}}\left(\bigcup_{\nu \in I_{n}^{*}} \mathcal{S}_{\mathfrak{g}, \nu}\right) \supseteq \bigcup_{\nu \in I_{n}^{*}} \operatorname{cl}_{\mathfrak{g}} \circ \operatorname{int}_{\mathfrak{g}}\left(\mathcal{S}_{\mathfrak{g}, \nu}\right)$
ii. $\operatorname{int}_{\mathfrak{g}} \circ \operatorname{cl}_{\mathfrak{g}}\left(\bigcup_{\nu \in I_{n}^{*}} \mathcal{S}_{\mathfrak{g}, \nu}\right) \supseteq \bigcup_{\nu \in I_{n}^{*}} \operatorname{int}_{\mathfrak{g}} \circ \operatorname{cl}_{\mathfrak{g}}\left(\mathcal{S}_{\mathfrak{g}, \nu}\right)$
iii. $\operatorname{cl}_{\mathfrak{g}} \circ \operatorname{int}_{\mathfrak{g}} \circ \mathrm{cl}_{\mathfrak{g}}\left(\bigcup_{\nu \in I_{n}^{*}} \mathcal{S}_{\mathfrak{g}, \nu}\right) \supseteq \bigcup_{\nu \in I_{n}^{*}} \operatorname{cl}_{\mathfrak{g}} \circ \operatorname{int}_{\mathfrak{g}} \circ \mathrm{cl}_{\mathfrak{g}}\left(\mathcal{S}_{\mathfrak{g}, \nu}\right)$ 
Proof. Since the relations

$$
\operatorname{cl}_{\mathfrak{g}}\left(\bigcup_{\nu \in I_{n}^{*}} \mathcal{S}_{\mathfrak{g}, \nu}\right)=\bigcup_{\nu \in I_{n}^{*}} \mathrm{cl}_{\mathfrak{g}}\left(\mathcal{S}_{\mathfrak{g}, \nu}\right), \quad \operatorname{int}_{\mathfrak{g}}\left(\bigcup_{\nu \in I_{n}^{*}} \mathcal{S}_{\mathfrak{g}, \nu}\right) \supseteq \bigcup_{\nu \in I_{n}^{*}} \operatorname{int}_{\mathfrak{g}}\left(\mathcal{S}_{\mathfrak{g}, \nu}\right)
$$

hold, it follows that

$$
\begin{aligned}
\operatorname{cl}_{\mathfrak{g}} \circ \operatorname{int}_{\mathfrak{g}}\left(\bigcup_{\nu \in I_{n}^{*}} \mathcal{S}_{\mathfrak{g}, \nu}\right) & \supseteq \operatorname{cl}_{\mathfrak{g}}\left(\bigcup_{\nu \in I_{n}^{*}} \operatorname{int}_{\mathfrak{g}}\left(\mathcal{S}_{\mathfrak{g}, \nu}\right)\right) \\
& =\bigcup_{\nu \in I_{n}^{*}} \operatorname{cl}_{\mathfrak{g}} \circ \operatorname{int}_{\mathfrak{g}}\left(\mathcal{S}_{\mathfrak{g}, \nu}\right) \\
\operatorname{int}_{\mathfrak{g}} \circ \operatorname{cl}_{\mathfrak{g}}\left(\bigcup_{\nu \in I_{n}^{*}} \mathcal{S}_{\mathfrak{g}, \nu}\right) & \supseteq \operatorname{int}_{\mathfrak{g}}\left(\bigcup_{\nu \in I_{n}^{*}} \operatorname{cl}_{\mathfrak{g}}\left(\mathcal{S}_{\mathfrak{g}, \nu}\right)\right) \\
\operatorname{cl}_{\mathfrak{g}} \circ \operatorname{int}_{\mathfrak{g}} \circ \operatorname{cl}_{\mathfrak{g}}\left(\bigcup_{\nu \in I_{n}^{*}} \mathcal{S}_{\mathfrak{g}, \nu}\right) & =\bigcup_{\nu \in I_{n}^{*}} \operatorname{cl}_{\mathfrak{g}} \circ \operatorname{int}_{\mathfrak{g}}\left(\mathcal{S}_{\mathfrak{g}, \nu}\right) \\
& \left.\supseteq \bigcup_{\nu \in I_{n}^{*}} \operatorname{cl}_{\mathfrak{g}} \circ \operatorname{int}_{\nu \in I_{n}^{*}} \operatorname{cl}_{\mathfrak{g}}\left(\mathcal{S}_{\mathfrak{g}, \nu}\right)\right)
\end{aligned}
$$

From the above proposition, it is obvious that their duals are

$$
\begin{aligned}
\operatorname{int}_{\mathfrak{g}} \circ \operatorname{cl}_{\mathfrak{g}}\left(\bigcap_{\nu \in I_{n}^{*}} \mathcal{S}_{\mathfrak{g}, \nu}\right) & \subseteq \bigcap_{\nu \in I_{n}^{*}} \operatorname{int}_{\mathfrak{g}} \circ \operatorname{cl}_{\mathfrak{g}}\left(\mathcal{S}_{\mathfrak{g}, \nu}\right) \\
\operatorname{cl}_{\mathfrak{g}} \circ \operatorname{int}_{\mathfrak{g}}\left(\bigcap_{\nu \in I_{n}^{*}} \mathcal{S}_{\mathfrak{g}, \nu}\right) & \subseteq \bigcap_{\nu \in I_{n}^{*}} \operatorname{cl}_{\mathfrak{g}} \circ \operatorname{int}_{\mathfrak{g}}\left(\mathcal{S}_{\mathfrak{g}, \nu}\right) \\
\operatorname{int}_{\mathfrak{g}} \circ \operatorname{cl}_{\mathfrak{g}} \circ \operatorname{int}_{\mathfrak{g}}\left(\bigcap_{\nu \in I_{n}^{*}} \mathcal{S}_{\mathfrak{g}, \nu}\right) & \subseteq \bigcap_{\nu \in I_{n}^{*}} \operatorname{int}_{\mathfrak{g}} \circ \operatorname{cl}_{\mathfrak{g}} \circ \operatorname{int}_{\mathfrak{g}}\left(\mathcal{S}_{\mathfrak{g}, \nu}\right)
\end{aligned}
$$

respectively. On this basis, we have the following corollary:

Corollary 3.7. Let $\mathbf{o p}_{\mathfrak{g}}(\cdot) \in \mathcal{L}_{\mathfrak{g}}[\Omega]$ be a $\mathfrak{g}$-operator in a $\mathcal{T}_{\mathfrak{g}}$-space $\mathfrak{T}_{\mathfrak{g}}$. If $\mathcal{S}_{\mathfrak{g}, 1}, \mathcal{S}_{\mathfrak{g}, 2}, \ldots, \mathcal{S}_{\mathfrak{g}, n} \subset \mathfrak{T}_{\mathfrak{g}}$ are $n \geq 1 \mathfrak{T}_{\mathfrak{g}}$-sets of the $\mathcal{T}_{\mathfrak{g}}$-space $\mathfrak{T}_{\mathfrak{g}}$, then,

$$
\begin{aligned}
\text { i. } \mathrm{op}_{\mathfrak{g}} \circ \neg \mathrm{op}_{\mathfrak{g}}\left(\bigcup_{\nu \in I_{n}^{*}} \mathcal{S}_{\mathfrak{g}, \nu}\right) \supseteq \bigcup_{\nu \in I_{n}^{*}} \mathrm{op}_{\mathfrak{g}} \circ \neg \mathrm{op}_{\mathfrak{g}}\left(\mathcal{S}_{\mathfrak{g}, \nu}\right) \\
i i . \quad \neg \mathrm{op}_{\mathfrak{g}} \circ \mathrm{op}_{\mathfrak{g}}\left(\bigcap_{\nu \in I_{n}^{*}} \mathcal{S}_{\mathfrak{g}, \nu}\right) \subseteq \bigcap_{\nu \in I_{n}^{*}} \neg \mathrm{op}_{\mathfrak{g}} \circ \mathrm{op}_{\mathfrak{g}}\left(\mathcal{S}_{\mathfrak{g}, \nu}\right)
\end{aligned}
$$

Theorem 3.8. If $\mathcal{S}_{\mathfrak{g}, 1}, \mathcal{S}_{\mathfrak{g}, 2}, \ldots, \mathcal{S}_{\mathfrak{g}, n} \in \mathfrak{g}$-S $\left[\mathfrak{T}_{\mathfrak{g}}\right]$ are $n \geq 1 \mathfrak{g}$ - $\mathfrak{T}_{\mathfrak{g}}$-sets of a class $\mathfrak{g}$-S $\left[\mathfrak{T}_{\mathfrak{g}}\right]$ in a $\mathcal{T}_{\mathfrak{g}}$-space $\mathfrak{T}_{\mathfrak{g}}$, then $\bigcup_{\nu \in I_{n}^{*}} \mathcal{S}_{\mathfrak{g}, \nu} \in \mathfrak{g}-\mathrm{S}\left[\mathfrak{T}_{\mathfrak{g}}\right]$.

Proof. The statement $\mathcal{S}_{\mathfrak{g}, \nu} \in \mathfrak{g}-\mathrm{S}\left[\mathfrak{T}_{\mathfrak{g}}\right]$ for every $\nu \in I_{n}^{*}$ is identical to the logical statement:

$$
\exists\left(\mathcal{O}_{\mathfrak{g}, \nu}, \mathcal{K}_{\mathfrak{g}, \nu}\right) \in \mathcal{T}_{\mathfrak{g}} \times \neg \mathcal{T}_{\mathfrak{g}}:\left(\mathcal{S}_{\mathfrak{g}, \nu} \subseteq \mathrm{op}_{\mathfrak{g}}\left(\mathcal{O}_{\mathfrak{g}, \nu}\right)\right) \vee\left(\mathcal{S}_{\mathfrak{g}, \nu} \supseteq \neg \mathrm{op}_{\mathfrak{g}}\left(\mathcal{K}_{\mathfrak{g}, \nu}\right)\right)
$$

On the other hand, if $\mathbf{o p}_{\mathfrak{g}}(\cdot) \in \mathcal{L}_{\mathfrak{g}}[\Omega]$ is a $\mathfrak{g}$-operator in the $\mathcal{T}_{\mathfrak{g}}$-space, then

$$
\begin{aligned}
\operatorname{op}_{\mathfrak{g}}\left(\bigcup_{\nu \in I_{n}^{*}} \mathcal{O}_{\mathfrak{g}, \nu}\right) & =\bigcup_{\nu \in I_{n}^{*}} \operatorname{op}_{\mathfrak{g}}\left(\mathcal{O}_{\mathfrak{g}, \nu}\right) \\
\neg \operatorname{op}_{\mathfrak{g}}\left(\bigcup_{\nu \in I_{n}^{*}} \mathcal{K}_{\mathfrak{g}, \nu}\right) & =\bigcup_{\nu \in I_{n}^{*}} \neg \operatorname{op}_{\mathfrak{g}}\left(\mathcal{K}_{\mathfrak{g}, \nu}\right)
\end{aligned}
$$

Consequently,

$$
\begin{aligned}
& \bigvee_{\nu \in I_{n}^{*}}\left(\left(\mathcal{S}_{\mathfrak{g}, \nu} \subseteq \operatorname{op}_{\mathfrak{g}}\left(\mathcal{O}_{\mathfrak{g}, \nu}\right)\right) \vee\left(\mathcal{S}_{\mathfrak{g}, \nu} \supseteq \neg \mathrm{op}_{\mathfrak{g}}\left(\mathcal{K}_{\mathfrak{g}, \nu}\right)\right)\right) \\
\Rightarrow \quad & \left(\left(\bigcup_{\nu \in I_{n}^{*}} \mathcal{S}_{\mathfrak{g}, \nu} \subseteq \bigcup_{\nu \in I_{n}^{*}} \mathrm{op}_{\mathfrak{g}}\left(\mathcal{O}_{\mathfrak{g}, \nu}\right)\right) \vee\left(\bigcup_{\nu \in I_{n}^{*}} \mathcal{S}_{\mathfrak{g}, \nu} \supseteq \bigcup_{\nu \in I_{n}^{*}} \neg \mathrm{op}_{\mathfrak{g}}\left(\mathcal{K}_{\mathfrak{g}, \nu}\right)\right)\right) \\
\Rightarrow \quad & \left(\left(\bigcup_{\nu \in I_{n}^{*}} \mathcal{S}_{\mathfrak{g}, \nu} \subseteq \mathrm{op}_{\mathfrak{g}}\left(\bigcup_{\nu \in I_{n}^{*}} \mathcal{O}_{\mathfrak{g}, \nu}\right)\right) \vee\left(\bigcup_{\nu \in I_{n}^{*}} \mathcal{S}_{\mathfrak{g}, \nu} \supseteq \neg \mathrm{op}_{\mathfrak{g}}\left(\bigcup_{\nu \in I_{n}^{*}} \mathcal{K}_{\mathfrak{g}, \nu}\right)\right)\right)
\end{aligned}
$$

But, $\bigcup_{\nu \in I_{n}^{*}} \mathcal{O}_{\mathfrak{g}, \nu} \in \mathcal{T}_{\mathfrak{g}}$ and $\bigcup_{\nu \in I_{n}^{*}} \mathcal{K}_{\mathfrak{g}, \nu} \in \neg \mathcal{T}_{\mathfrak{g}}$. Hence, $\bigcup_{\nu \in I_{n}^{*}} \mathcal{S}_{\mathfrak{g}, \nu} \in \mathfrak{g}-\mathrm{S}\left[\mathfrak{T}_{\mathfrak{g}}\right]$. 
Theorem 3.9. If $\mathcal{S}_{\mathfrak{g}, 1}, \mathcal{S}_{\mathfrak{g}, 2}, \ldots, \mathcal{S}_{\mathfrak{g}, n} \in \mathfrak{g}$-S $\left[\mathfrak{T}_{\mathfrak{g}}\right]$ are $n \geq 1 \mathfrak{g}$ - $\mathfrak{T}_{\mathfrak{g}}$-sets of a class $\mathfrak{g}$-S $\left[\mathfrak{T}_{\mathfrak{g}}\right]$ in a $\mathcal{T}_{\mathfrak{g}}$-space $\mathfrak{T}_{\mathfrak{g}}$, then

$$
\left(\bigcap_{\nu \in I_{n}^{*}} \mathcal{S}_{\mathfrak{g}, \nu} \in \mathfrak{g}-\mathrm{S}\left[\mathfrak{T}_{\mathfrak{g}}\right]\right) \vee\left(\bigcap_{\nu \in I_{n}^{*}} \mathcal{S}_{\mathfrak{g}, \nu} \notin \mathfrak{g}-\mathrm{S}\left[\mathfrak{T}_{\mathfrak{g}}\right]\right)
$$

Proof. Because, $\mathcal{S}_{\mathfrak{g}, 1}, \mathcal{S}_{\mathfrak{g}, 2}, \ldots, \mathcal{S}_{\mathfrak{g}, n} \in \mathfrak{g}-\mathrm{S}\left[\mathfrak{T}_{\mathfrak{g}}\right]$ by hypothesis, the trueness of $\bigcap_{\nu \in I_{n}^{*}} \mathcal{S}_{\mathfrak{g}, \nu} \in \mathfrak{g}-\mathrm{S}\left[\mathfrak{T}_{\mathfrak{g}}\right]$ and $\bigcap_{\nu \in I_{n}^{*}} \mathcal{S}_{\mathfrak{g}, \nu} \notin \mathfrak{g}-\mathrm{S}\left[\mathfrak{T}_{\mathfrak{g}}\right]$ evidently depend on the following property:

$$
\bigwedge_{\nu \in I_{n}^{*}}\left(\left(\mathcal{S}_{\mathfrak{g}, \nu} \subseteq \mathrm{op}_{\mathfrak{g}}\left(\mathcal{O}_{\mathfrak{g}, \nu}\right)\right) \vee\left(\mathcal{S}_{\mathfrak{g}, \nu} \supseteq \neg \mathrm{op}_{\mathfrak{g}}\left(\mathcal{K}_{\mathfrak{g}, \nu}\right)\right)\right)
$$

where $\left(\mathcal{O}_{\mathfrak{g}, \nu}, \mathcal{K}_{\mathfrak{g}, \nu}\right) \in \mathcal{T}_{\mathfrak{g}} \times \neg \mathcal{T}_{\mathfrak{g}}$ for every $\nu \in I_{n}^{*}$. Furthermore, because the $\mathfrak{g}-\mathfrak{T}_{\mathfrak{g}}$-set-theoretic operations concern finite intersections, it suffices to prove the theorem for $n=2$. Set the first property preceding $\vee$ to $\mathrm{P}(\nu)$ and that following $\vee$ to $\mathrm{Q}(\nu)$. Then, its decomposition gives

$$
\begin{aligned}
\bigwedge_{\nu \in I_{2}^{*}}(\mathrm{P}(\nu) \vee \mathrm{Q}(\nu)) & =\left(\bigwedge_{\nu \in I_{2}^{*}} \mathrm{P}(\nu)\right) \vee\left(\bigwedge_{\nu \in I_{2}^{*}} \mathrm{Q}(\nu)\right) \\
& =(\mathrm{P}(1) \wedge \mathrm{Q}(2)) \vee(\mathrm{P}(2) \wedge \mathrm{Q}(1))
\end{aligned}
$$

If $\mathcal{S}_{\mathfrak{g}, 1}, \mathcal{S}_{\mathfrak{g}, 2} \in \mathfrak{g}$-S $\left[\mathfrak{T}_{\mathfrak{g}}\right]$ are both $\mathfrak{g}-\mathfrak{T}_{\mathfrak{g}}$-open sets then $\bigwedge_{\nu \in I_{2}^{*}} \mathrm{P}(\nu)$ is true, and if they are both $\mathfrak{g}$ - $\mathcal{T}_{\mathfrak{g}}$-closed sets then $\bigwedge_{\nu \in I_{2}^{*}} \mathrm{Q}(\nu)$ is true. In these two cases, $\bigcap_{\nu \in I_{2}^{*}} \mathcal{S}_{\mathfrak{g}, \nu} \in \mathfrak{g}-\mathrm{S}\left[\mathfrak{T}_{\mathfrak{g}}\right]$. Because, in general, there does not necessarily exists $\mathfrak{g}$ - $\mathfrak{T}_{\mathfrak{g}}$-set which is simultaneously $\mathfrak{g}$ - $\mathcal{T}_{\mathfrak{g}}$-open and $\mathfrak{g}$ - $\mathcal{T}_{\mathfrak{g}}$-closed, both $\mathrm{P}(1) \wedge \mathrm{Q}(2)$ and $\mathrm{P}(2) \wedge \mathrm{Q}(1)$ are untrue; thus, $\bigcap_{\nu \in I_{2}^{*}} \mathcal{S}_{\mathfrak{g}, \nu} \notin \mathfrak{g}-\mathrm{S}\left[\mathfrak{T}_{\mathfrak{g}}\right]$.

Theorem 3.10. Let $\mathcal{S}_{\mathfrak{g}} \subset \mathfrak{T}_{\mathfrak{g}}$ be a $\mathfrak{T}_{\mathfrak{g}}$-set and let $\mathbf{o p}_{\mathfrak{g}}(\cdot) \in \mathcal{L}_{\mathfrak{g}}[\Omega]$ be a $\mathfrak{g}$-operator in a $\mathcal{T}_{\mathfrak{g}}$-space. If $\mathcal{S}_{\mathfrak{g}} \in \mathfrak{g}-\mathrm{S}\left[\mathfrak{T}_{\mathfrak{g}}\right]$, then

$$
\left(\mathrm{op}_{\mathfrak{g}}\left(\mathcal{S}_{\mathfrak{g}}\right) \in \mathfrak{g}-\mathrm{S}\left[\mathfrak{T}_{\mathfrak{g}}\right]\right) \vee\left(\neg \mathrm{op}_{\mathfrak{g}}\left(\mathcal{S}_{\mathfrak{g}}\right) \in \mathfrak{g}-\mathrm{S}\left[\mathfrak{T}_{\mathfrak{g}}\right]\right)
$$

Proof. Let $\mathcal{S}_{\mathfrak{g}} \in \mathfrak{g}-\mathrm{S}\left[\mathfrak{T}_{\mathfrak{g}}\right]$. Then, $\left(\mathcal{S}_{\mathfrak{g}} \subseteq \operatorname{op}_{\mathfrak{g}}\left(\mathcal{O}_{\mathfrak{g}}\right)\right) \vee\left(\mathcal{S}_{\mathfrak{g}} \supseteq \neg \operatorname{op}_{\mathfrak{g}}\left(\mathcal{K}_{\mathfrak{g}}\right)\right)$ for some pair $\left(\mathcal{O}_{\mathfrak{g}}, \mathcal{K}_{\mathfrak{g}}\right) \in$ $\mathcal{T}_{\mathfrak{g}} \times \neg \mathcal{T}_{\mathfrak{g}}$ of $\mathcal{T}_{\mathfrak{g}}$-open and $\mathcal{T}_{\mathfrak{g}}$-closed sets relative to $\mathcal{T}_{\mathfrak{g}}$. Consequently, op $\mathfrak{g}_{\mathfrak{g}}\left(\mathcal{S}_{\mathfrak{g}}\right) \subseteq \mathrm{op}_{\mathfrak{g}} \circ \mathrm{op}_{\mathfrak{g}}\left(\mathcal{O}_{\mathfrak{g}}\right)$ or $\neg \mathrm{op}_{\mathfrak{g}}\left(\mathcal{S}_{\mathfrak{g}}\right) \supseteq \neg \mathrm{op}_{\mathfrak{g}} \circ \neg \mathrm{op}_{\mathfrak{g}}\left(\mathcal{K}_{\mathfrak{g}}\right)$. But, $\mathrm{op}_{\mathfrak{g}} \circ \mathrm{op}_{\mathfrak{g}}\left(\mathcal{O}_{\mathfrak{g}}\right) \subseteq \mathrm{op}_{\mathfrak{g}}\left(\mathcal{O}_{\mathfrak{g}}\right)$ and $\neg \mathrm{op}_{\mathfrak{g}} \circ \neg \mathrm{op}_{\mathfrak{g}}\left(\mathcal{K}_{\mathfrak{g}}\right) \supseteq \neg \mathrm{op}_{\mathfrak{g}}\left(\mathcal{K}_{\mathfrak{g}}\right)$. Thus, there follows that $\operatorname{op}_{\mathfrak{g}}\left(\mathcal{S}_{\mathfrak{g}}\right) \subseteq \operatorname{op}_{\mathfrak{g}}\left(\mathcal{O}_{\mathfrak{g}}\right)$ or $\neg \mathrm{op}_{\mathfrak{g}}\left(\mathcal{S}_{\mathfrak{g}}\right) \supseteq \neg \operatorname{op}_{\mathfrak{g}}\left(\mathcal{K}_{\mathfrak{g}}\right)$. Hence, $\operatorname{op}_{\mathfrak{g}}\left(\mathcal{S}_{\mathfrak{g}}\right) \in \mathfrak{g}-\mathrm{S}\left[\mathfrak{T}_{\mathfrak{g}}\right]$ or $\neg \operatorname{op}_{\mathfrak{g}}\left(\mathcal{S}_{\mathfrak{g}}\right) \in \mathfrak{g}-\mathrm{S}\left[\mathfrak{T}_{\mathfrak{g}}\right]$.

Proposition 3.11. Let $\mathcal{S}_{\mathfrak{g}} \in \mathfrak{g}-\mathrm{S}\left[\mathfrak{T}_{\mathfrak{g}}\right]$ in a $\mathcal{T}_{\mathfrak{g}}$-space $\mathfrak{T}_{\mathfrak{g}}$ and suppose the logical statement

$$
\left(\exists \mathcal{R}_{\mathfrak{g}} \subset \mathfrak{T}_{\mathfrak{g}}\right)\left[\left(\mathcal{R}_{\mathfrak{g}} \subseteq \mathrm{op}_{\mathfrak{g}}\left(\mathcal{S}_{\mathfrak{g}}\right)\right) \vee\left(\mathcal{R}_{\mathfrak{g}} \supseteq \neg \mathrm{op}_{\mathfrak{g}}\left(\mathcal{S}_{\mathfrak{g}}\right)\right)\right]
$$

holds, then $\mathcal{R}_{\mathfrak{g}} \in \mathfrak{g}-\mathrm{S}\left[\mathfrak{T}_{\mathfrak{g}}\right]$.

Proof. Let there exists a $\mathfrak{T}_{\mathfrak{g}}$-set $\mathcal{R}_{\mathfrak{g}} \subset \mathfrak{T}_{\mathfrak{g}}$ such that $\mathcal{R}_{\mathfrak{g}} \subseteq$ op $_{\mathfrak{g}}\left(\mathcal{S}_{\mathfrak{g}}\right)$ or $\mathcal{R}_{\mathfrak{g}} \supseteq \neg \operatorname{op}_{\mathfrak{g}}\left(\mathcal{S}_{\mathfrak{g}}\right)$. But $\mathcal{S}_{\mathfrak{g}} \in$ $\mathfrak{g}-\mathrm{S}\left[\mathfrak{T}_{\mathfrak{g}}\right]$ implies op $\mathfrak{g}_{\mathfrak{g}}\left(\mathcal{S}_{\mathfrak{g}}\right) \in \mathfrak{g}-\mathrm{S}\left[\mathfrak{T}_{\mathfrak{g}}\right]$ or $\neg \mathrm{op}_{\mathfrak{g}}\left(\mathcal{S}_{\mathfrak{g}}\right) \in \mathfrak{g}-\mathrm{S}\left[\mathfrak{T}_{\mathfrak{g}}\right]$. Thus, $\mathcal{R}_{\mathfrak{g}} \in \mathfrak{g}-\mathrm{S}\left[\mathfrak{T}_{\mathfrak{g}}\right]$.

Corollary 3.12. Let $\mathfrak{T}_{\mathfrak{g}}$ be a $\mathcal{T}_{\mathfrak{g}}$-space. If $\mathfrak{g}-\mathrm{S}\left[\mathfrak{T}_{\mathfrak{g}}\right]=\mathfrak{g}-\mathrm{O}\left[\mathfrak{T}_{\mathfrak{g}}\right] \cup \mathfrak{g}-\mathrm{K}\left[\mathfrak{T}_{\mathfrak{g}}\right]$ denotes a class of $\mathfrak{g}-\mathfrak{T}_{\mathfrak{g}}$-open and $\mathfrak{g}$ - $\mathfrak{T}_{\mathfrak{g}}$-closed sets, and $\mathrm{S}\left[\mathfrak{T}_{\mathfrak{g}}\right]=\mathrm{O}\left[\mathfrak{T}_{\mathfrak{g}}\right] \cup \mathrm{K}\left[\mathfrak{T}_{\mathfrak{g}}\right]$ denotes a class of $\mathfrak{T}_{\mathfrak{g}}$-open and $\mathfrak{T}_{\mathfrak{g}}$-closed sets, then

$$
\mathfrak{g}-\mathrm{S}\left[\mathfrak{T}_{\mathfrak{g}}\right] \supseteq \mathfrak{g}-\mathrm{O}\left[\mathfrak{T}_{\mathfrak{g}}\right] \cup \mathfrak{g}-\mathrm{K}\left[\mathfrak{T}_{\mathfrak{g}}\right] \supseteq \mathrm{O}\left[\mathfrak{T}_{\mathfrak{g}}\right] \cup \mathrm{K}\left[\mathfrak{T}_{\mathfrak{g}}\right] \supseteq \mathrm{S}\left[\mathfrak{T}_{\mathfrak{g}}\right]
$$

An important remark should be pointed out at this stage.

Remark 3.13. The converse of the statement "if $\mathcal{S}_{\mathfrak{g}} \in \mathrm{S}\left[\mathfrak{T}_{\mathfrak{g}}\right]$ then $\mathcal{S}_{\mathfrak{g}} \in \mathfrak{g}-\mathrm{S}\left[\mathfrak{T}_{\mathfrak{g}}\right]$ " is obviously untrue. Because, the negation of this statement gives

$$
\left(\mathcal{S}_{\mathfrak{g}} \in \mathrm{S}\left[\mathfrak{T}_{\mathfrak{g}}\right]\right) \wedge\left(\neg\left(\mathcal{S}_{\mathfrak{g}} \in \mathfrak{g}-\mathrm{S}\left[\mathfrak{T}_{\mathfrak{g}}\right]\right)\right)
$$

which is an untrue statements. 
Theorem 3.14. Let $\mathfrak{T}_{\mathfrak{g}}$ be a $\mathcal{T}_{\mathfrak{g}}$-space. If $\mathcal{S}_{\mathfrak{g}} \subset \mathfrak{T}_{\mathfrak{g}}$, then

$$
\mathcal{S}_{\mathfrak{g}} \in \mathfrak{g}-\mathrm{S}\left[\mathfrak{T}_{\mathfrak{g}}\right] \Leftrightarrow\left(\mathcal{S}_{\mathfrak{g}} \subseteq \mathrm{op}_{\mathfrak{g}} \circ \neg \mathrm{op}_{\mathfrak{g}}\left(\mathcal{S}_{\mathfrak{g}}\right)\right) \vee\left(\mathcal{S}_{\mathfrak{g}} \supseteq \neg \mathrm{op}_{\mathfrak{g}} \circ \mathrm{op}_{\mathfrak{g}}\left(\mathcal{S}_{\mathfrak{g}}\right)\right)
$$

Proof.

$(\Leftarrow)$ : Let

$$
\left(\mathcal{S}_{\mathfrak{g}} \subseteq \mathrm{op}_{\mathfrak{g}} \circ \neg \mathrm{op}_{\mathfrak{g}}\left(\mathcal{S}_{\mathfrak{g}}\right)\right) \vee\left(\mathcal{S}_{\mathfrak{g}} \supseteq \neg \mathrm{op}_{\mathfrak{g}} \circ \mathrm{op}_{\mathfrak{g}}\left(\mathcal{S}_{\mathfrak{g}}\right)\right)
$$

Then, the substitution of $\neg \mathrm{op}_{\mathfrak{g}}\left(\mathcal{S}_{\mathfrak{g}}\right)=\mathcal{O}_{\mathfrak{g}}$ in the logical statement preceding $\vee$ and $\operatorname{op}_{\mathfrak{g}}\left(\mathcal{S}_{\mathfrak{g}}\right)=\mathcal{K}_{\mathfrak{g}}$ in that following $\vee$ gives $\left(\mathcal{S}_{\mathfrak{g}} \subseteq \mathrm{op}_{\mathfrak{g}}\left(\mathcal{O}_{\mathfrak{g}}\right)\right) \vee\left(\mathcal{S}_{\mathfrak{g}} \supseteq \neg \mathrm{op}_{\mathfrak{g}}\left(\mathcal{K}_{\mathfrak{g}}\right)\right)$.

$(\Rightarrow)$ : Let $\mathcal{S}_{\mathfrak{g}} \in \mathfrak{g}-\mathrm{S}\left[\mathfrak{T}_{\mathfrak{g}}\right]$. Then, $\left(\mathcal{S}_{\mathfrak{g}} \subseteq \operatorname{op}_{\mathfrak{g}}\left(\mathcal{O}_{\mathfrak{g}}\right)\right) \vee\left(\mathcal{S}_{\mathfrak{g}} \supseteq \neg \mathrm{op}_{\mathfrak{g}}\left(\mathcal{K}_{\mathfrak{g}}\right)\right)$. Consequently, substituting $\mathcal{O}_{\mathfrak{g}}=\neg \mathrm{op}_{\mathfrak{g}}\left(\mathcal{S}_{\mathfrak{g}}\right)$ in the logical statement preceding $\vee$ and $\mathcal{K}_{\mathfrak{g}}=\operatorname{op}_{\mathfrak{g}}\left(\mathcal{S}_{\mathfrak{g}}\right)$ in that following $\vee$, the required logical statement at once follows, which proves the theorem.

The class $\mathfrak{g}-\mathrm{S}\left[\mathfrak{T}_{\mathfrak{g}}\right]$ forms a $\mathfrak{g}$-topology on $\Omega$, which will be denoted by $\mathcal{T}_{\mathfrak{g}-\mathrm{s}}$.

Theorem 3.15. Let $\mathfrak{g}-\mathrm{S}\left[\mathfrak{T}_{\mathfrak{g}}\right]$ be a given $\mathfrak{g}$-class in a $\mathcal{T}_{\mathfrak{g}}$-space $\mathfrak{T}_{\mathfrak{g}}$. Then, the one-valued map $\mathcal{T}_{\mathfrak{g}-\mathrm{S}}$ : $\mathfrak{g}-\mathrm{S}\left[\mathfrak{T}_{\mathfrak{g}}\right] \longrightarrow \mathfrak{g}-\mathrm{S}\left[\mathfrak{T}_{\mathfrak{g}}\right]$ forms a $\mathfrak{g}$-topology on $\Omega$ in the $\mathcal{T}_{\mathfrak{g}}$-space.

Proof. By definition, $\left(\emptyset=\operatorname{op}_{\mathfrak{g}}(\emptyset)\right) \vee\left(\emptyset=\neg \operatorname{op}_{\mathfrak{g}}(\emptyset)\right)$. Since, either $\operatorname{op}_{\mathfrak{g}}(\emptyset) \subseteq \operatorname{op}_{\mathfrak{g}}\left(\mathcal{O}_{\mathfrak{g}}\right)$ or $\neg \operatorname{op}_{\mathfrak{g}}(\emptyset) \supseteq$ $\neg \mathrm{op}_{\mathfrak{g}}\left(\mathcal{K}_{\mathfrak{g}}\right)$ holds, where $\mathcal{O}_{\mathfrak{g}}, \mathcal{K}_{\mathfrak{g}} \subset \mathfrak{T}_{\mathfrak{g}}$, respectively, are some $\mathcal{T}_{\mathfrak{g}}$-open and $\mathcal{T}_{\mathfrak{g}}$-closed sets in $\mathfrak{T}_{\mathfrak{g}}$, it follows that $\emptyset \in \mathfrak{g}-\mathrm{S}\left[\mathfrak{T}_{\mathfrak{g}}\right]$ and, hence, $\mathcal{T}_{\mathfrak{g}-\mathrm{S}}(\emptyset)=\emptyset$. Let $\mathcal{S}_{\mathfrak{g}} \in \mathfrak{g}-\mathrm{S}\left[\mathfrak{T}_{\mathfrak{g}}\right]$. Then, since $\mathfrak{g}-\mathrm{S}\left[\mathfrak{T}_{\mathfrak{g}}\right] \subseteq \mathfrak{g}-\mathrm{S}\left[\mathfrak{T}_{\mathfrak{g}}\right]$, it follows that $\mathcal{S}_{\mathfrak{g}}$ is a superset of $\mathcal{T}_{\mathfrak{g}-\mathrm{S}}\left(\mathcal{S}_{\mathfrak{g}}\right)$. Hence, $\mathcal{T}_{\mathfrak{g}-\mathrm{S}}\left(\mathcal{S}_{\mathfrak{g}}\right) \subseteq \mathcal{S}_{\mathfrak{g}}$. Let $\mathcal{S}_{\mathfrak{g}, 1}, \mathcal{S}_{\mathfrak{g}, 2}, \ldots$ be $\mathfrak{T}_{\mathfrak{g}}$-sets satisfying, for every $\nu \in I_{\infty}^{*}, \mathcal{S}_{\mathfrak{g}, \nu}$. Then, there exist classes $\left\{\mathcal{O}_{\mathfrak{g}, \nu} \in \mathcal{T}_{\mathfrak{g}}: \quad \nu \in I_{\infty}^{*}\right\}$ and $\left\{\mathcal{K}_{\mathfrak{g}, \nu} \in \neg \mathcal{T}_{\mathfrak{g}}: \quad \nu \in I_{\infty}^{*}\right\}$, respectively, of $\mathcal{T}_{\mathfrak{g}}$-open and $\mathcal{T}_{\mathfrak{g}}$-closed sets such that

$$
\left(\bigcup_{\nu \in I_{\infty}^{*}} \mathcal{S}_{\mathfrak{g}, \nu} \subseteq \mathrm{op}_{\mathfrak{g}}\left(\bigcup_{\nu \in I_{\infty}^{*}} \mathcal{O}_{\mathfrak{g}, \nu}\right)\right) \vee\left(\bigcup_{\nu \in I_{\infty}^{*}} \mathcal{S}_{\mathfrak{g}, \nu} \supseteq \neg \mathrm{op}_{\mathfrak{g}}\left(\bigcup_{\nu \in I_{\infty}^{*}} \mathcal{K}_{\mathfrak{g}, \nu}\right)\right)
$$

a relation established on the following expressions:

$$
\begin{aligned}
\bigcup_{\nu \in I_{\infty}^{*}} \operatorname{op}_{\mathfrak{g}}\left(\mathcal{O}_{\mathfrak{g}, \nu}\right) & =\operatorname{op}_{\mathfrak{g}}\left(\bigcup_{\nu \in I_{\infty}^{*}} \mathcal{O}_{\mathfrak{g}, \nu}\right) \\
\bigcup_{\nu \in I_{\infty}^{*}} \neg \mathrm{op}_{\mathfrak{g}}\left(\mathcal{K}_{\mathfrak{g}, \nu}\right) & =\neg \operatorname{op}_{\mathfrak{g}}\left(\bigcup_{\nu \in I_{\infty}^{*}} \mathcal{K}_{\mathfrak{g}, \nu}\right)
\end{aligned}
$$

Consequently, $\bigcup_{\nu \in I_{\infty}^{*}} \mathcal{S}_{\mathfrak{g}, \nu} \in \mathfrak{g}-\mathrm{S}\left[\mathfrak{T}_{\mathfrak{g}}\right]$, since $\bigcup_{\nu \in I_{\infty}^{*}} \mathcal{O}_{\mathfrak{g}, \nu} \in \mathcal{T}_{\mathfrak{g}}$ is a $\mathcal{T}_{\mathfrak{g}}$-open set and $\bigcup_{\nu \in I_{\infty}^{*}} \mathcal{O}_{\mathfrak{g}, \nu} \in \neg \mathcal{T}_{\mathfrak{g}}$ is a $\mathcal{T}_{\mathfrak{g}}$-closed set. Hence,

$$
\mathcal{T}_{\mathfrak{g}-\mathrm{S}}\left(\bigcup_{\nu \in I_{\infty}^{*}} \mathcal{S}_{\mathfrak{g}, \nu}\right)=\bigcup_{\nu \in I_{\infty}^{*}} \mathcal{T}_{\mathfrak{g}-\mathrm{S}}\left(\mathcal{S}_{\mathfrak{g}, \nu}\right)
$$

An immediate consequence of the above theorem is the following corollary.

Corollary 3.16. Let a $\mathfrak{T}_{\mathfrak{g}}$ be a $\mathcal{T}_{\mathfrak{g}}$-space. Then, the structure $\left(\Omega, \mathcal{T}_{\mathfrak{g}-\mathrm{S}}\right)$, where $\mathcal{T}_{\mathfrak{g}-\mathrm{S}}: \mathfrak{g}-\mathrm{S}\left[\mathfrak{T}_{\mathfrak{g}}\right] \longrightarrow$ $\mathfrak{g}-\mathrm{S}\left[\mathfrak{T}_{\mathfrak{g}}\right]$, is a $\mathcal{T}_{\mathfrak{g}}$-space.

To condense the set-builder notation describing the classes $\mathfrak{g}-\mathrm{S}\left[\mathfrak{T}_{\mathfrak{g}}\right]$ and then classify it into subclasses, predicates must be introduced, and the choice made is to consider the so-called Boolean-valued functions on $\mathfrak{T}_{\mathfrak{g}} \times \mathcal{T}_{\mathfrak{g}} \cup \neg \mathcal{T}_{\mathfrak{g}} \times \mathcal{L}_{\mathfrak{g}}[\Omega] \times\{\subseteq, \supseteq\}$, the definition of which are given below.

Definition 3.17. Let $\left(\mathcal{S}_{\mathfrak{g}}, \mathcal{O}_{\mathfrak{g}}, \mathcal{K}_{\mathfrak{g}}\right) \in \mathfrak{T}_{\mathfrak{g}} \times \mathcal{T}_{\mathfrak{g}} \times \neg \mathcal{T}_{\mathfrak{g}}$ and let $\mathbf{o p}_{\mathfrak{g}}(\cdot) \in \mathcal{L}_{\mathfrak{g}}[\Omega]$ be a $\mathfrak{g}$-operator in a $\mathcal{T}_{\mathfrak{g}}$-space $\mathfrak{T}_{\mathfrak{g}}$. The first two predicates

$$
\begin{aligned}
& \mathrm{P}_{\mathfrak{g}}\left(\mathcal{S}_{\mathfrak{g}}, \mathcal{O}_{\mathfrak{g}} ; \mathbf{o p}_{\mathfrak{g}}(\cdot) ; \subseteq\right):=\left(\exists \mathcal{O}_{\mathfrak{g}}, \mathrm{op}_{\mathfrak{g}}(\cdot)\right)\left(\mathcal{S}_{\mathfrak{g}} \subseteq \mathrm{op}_{\mathfrak{g}}\left(\mathcal{O}_{\mathfrak{g}}\right)\right) \\
& \mathrm{P}_{\mathfrak{g}}\left(\mathcal{S}_{\mathfrak{g}}, \mathcal{K}_{\mathfrak{g}} ; \mathbf{o p}_{\mathfrak{g}}(\cdot) ; \supseteq\right):=\left(\exists \mathcal{K}_{\mathfrak{g}}, \neg \mathrm{op}_{\mathfrak{g}}(\cdot)\right)\left(\mathcal{S}_{\mathfrak{g}} \supseteq \neg \mathrm{op}_{\mathfrak{g}}\left(\mathcal{K}_{\mathfrak{g}}\right)\right) \\
& \mathrm{P}_{\mathfrak{g}}\left(\mathcal{S}_{\mathfrak{g}}, \mathcal{O}_{\mathfrak{g}}, \mathcal{K}_{\mathfrak{g}} ; \mathbf{o p}_{\mathfrak{g}}(\cdot) ; \subseteq, \supseteq\right):=\mathrm{P}_{\mathfrak{g}}\left(\mathcal{S}_{\mathfrak{g}}, \mathcal{O}_{\mathfrak{g}} ; \mathbf{o p}_{\mathfrak{g}}(\cdot) ; \subseteq\right) \\
& \vee \mathrm{P}_{\mathfrak{g}}\left(\mathcal{S}_{\mathfrak{g}}, \mathcal{K}_{\mathfrak{g}} ; \mathbf{o p}_{\mathfrak{g}}(\cdot) ; \supseteq\right)
\end{aligned}
$$

are called a Boolean-valued functions on $\mathfrak{T}_{\mathfrak{g}} \times \mathcal{T}_{\mathfrak{g}} \cup \neg \mathcal{T}_{\mathfrak{g}} \times \mathcal{L}_{\mathfrak{g}}[\Omega] \times\{\subseteq, \supseteq\}$. 
In this respect, $\mathfrak{g}-\mathrm{S}\left[\mathfrak{T}_{\mathfrak{g}}\right]:=\left\{\mathcal{S}_{\mathfrak{g}} \subset \mathfrak{T}_{\mathfrak{g}}: \mathrm{P}_{\mathfrak{g}}\left(\mathcal{S}_{\mathfrak{g}}, \mathcal{O}_{\mathfrak{g}}, \mathcal{K}_{\mathfrak{g}} ; \mathbf{o p}_{\mathfrak{g}}(\cdot) ; \subseteq, \supseteq\right)\right\}$. Moreover, employing the set-builder notations, the class of $\mathfrak{g}$ - $\mathfrak{T}_{\mathfrak{g}}$-open and $\mathfrak{g}$ - $\mathfrak{T}_{\mathfrak{g}}$-closed sets, denoted by $\mathfrak{g}-\mathrm{O}\left[\mathfrak{T}_{\mathfrak{g}}\right]$ and $\mathfrak{g}-\mathrm{K}\left[\mathfrak{T}_{\mathfrak{g}}\right]$, respectively, may then be defined as thus:

Definition 3.18. Let $\mathfrak{T}_{\mathfrak{g}}$ be a $\mathcal{T}_{\mathfrak{g}}$-space. The classes

$$
\begin{aligned}
\mathfrak{g}-\mathrm{O}\left[\mathfrak{T}_{\mathfrak{g}}\right] & :=\left\{\mathcal{S}_{\mathfrak{g}} \subset \mathfrak{T}_{\mathfrak{g}}: \mathrm{P}_{\mathfrak{g}}\left(\mathcal{S}_{\mathfrak{g}}, \mathcal{O}_{\mathfrak{g}} ; \mathbf{o p}_{\mathfrak{g}}(\cdot) ; \subseteq\right)\right\} \\
\mathfrak{g}-\mathrm{K}\left[\mathfrak{T}_{\mathfrak{g}}\right]:= & \left\{\mathcal{S}_{\mathfrak{g}} \subset \mathfrak{T}_{\mathfrak{g}}: \mathrm{P}_{\mathfrak{g}}\left(\mathcal{S}_{\mathfrak{g}}, \mathcal{K}_{\mathfrak{g}} ; \mathbf{o p}_{\mathfrak{g}}(\cdot) ; \supseteq\right)\right\}
\end{aligned}
$$

respectively, such that $\mathfrak{g}-\mathrm{S}\left[\mathfrak{T}_{\mathfrak{g}}\right]=\mathfrak{g}-\mathrm{O}\left[\mathfrak{T}_{\mathfrak{g}}\right] \cup \mathfrak{g}-\mathrm{K}\left[\mathfrak{T}_{\mathfrak{g}}\right]$, denote the families of all $\mathfrak{g}-\mathfrak{T}_{\mathfrak{g}}$-open and $\mathfrak{g}-\mathfrak{T}_{\mathfrak{g}}$ closed sets in $\mathfrak{T}_{\mathfrak{g}}$.

It is interesting to demonstrate their usefulness. In this direction, let us prove in a different way that $\mathfrak{g}-\mathfrak{T}_{\mathfrak{g}}$-set-theoretic operations is closed under arbitrary unions.

$$
\begin{aligned}
& \mathrm{P}_{\mathfrak{g}}\left(\mathcal{S}_{\mathfrak{g}}, \mathcal{O}_{\mathfrak{g}} ; \mathbf{o p}_{\mathfrak{g}}(\cdot) ; \subseteq\right):=\left(\exists \mathcal{O}_{\mathfrak{g}}, \mathrm{op}_{\mathfrak{g}}(\cdot)\right)\left(\mathcal{S}_{\mathfrak{g}} \subseteq \mathrm{op}_{\mathfrak{g}}\left(\mathcal{O}_{\mathfrak{g}}\right)\right) \\
& \mathrm{P}_{\mathfrak{g}}\left(\mathcal{S}_{\mathfrak{g}}, \mathcal{K}_{\mathfrak{g}} ; \mathbf{o p}_{\mathfrak{g}}(\cdot) ; \supseteq\right):=\left(\exists \mathcal{K}_{\mathfrak{g}}, \neg \mathrm{op}_{\mathfrak{g}}(\cdot)\right)\left(\mathcal{S}_{\mathfrak{g}} \supseteq \neg \mathrm{op}_{\mathfrak{g}}\left(\mathcal{K}_{\mathfrak{g}}\right)\right) \\
& \mathrm{P}_{\mathfrak{g}}\left(\mathcal{S}_{\mathfrak{g}}, \mathcal{O}_{\mathfrak{g}}, \mathcal{K}_{\mathfrak{g}} ; \mathbf{o p}_{\mathfrak{g}}(\cdot) ; \subseteq, \supseteq\right):=\mathrm{P}_{\mathfrak{g}}\left(\mathcal{S}_{\mathfrak{g}}, \mathcal{O}_{\mathfrak{g}} ; \mathbf{o p}_{\mathfrak{g}}(\cdot) ; \subseteq\right) \\
& \vee \mathrm{P}_{\mathfrak{g}}\left(\mathcal{S}_{\mathfrak{g}}, \mathcal{K}_{\mathfrak{g}} ; \mathbf{o p}_{\mathfrak{g}}(\cdot) ; \supseteq\right)
\end{aligned}
$$

Theorem 3.19. If $\left\{\mathcal{S}_{\mathfrak{g}, \nu} \in \mathfrak{g}-\mathrm{O}\left[\mathfrak{T}_{\mathfrak{g}}\right]: \nu \in I_{n}^{*}\right\}$ and $\left\{\mathcal{S}_{\mathfrak{g}, \nu} \in \mathfrak{g}-\mathrm{K}\left[\mathfrak{T}_{\mathfrak{g}}\right]: \nu \in I_{n}^{*}\right\}$, respectively, are finite collections of $\mathfrak{g}-\mathfrak{T}_{\mathfrak{g}}$-open and $\mathfrak{g}$ - $\mathfrak{T}_{\mathfrak{g}}$-closed sets in a $\mathcal{T}_{\mathfrak{g}}$-space $\mathfrak{T}_{\mathfrak{g}}$, then

$$
\begin{aligned}
& \bigcup_{\mu \in I_{n}^{*}}\left\{\xi \in \mathfrak{T}_{\mathfrak{g}}:\left(\exists \nu \in I_{\mu}^{*}\right)\left(\xi \in \mathcal{S}_{\mathfrak{g}, \nu} \in \mathfrak{g}-\mathrm{O}\left[\mathfrak{T}_{\mathfrak{g}}\right]\right)\right\} \subseteq \mathfrak{g}-\mathrm{O}\left[\mathfrak{T}_{\mathfrak{g}}\right] \\
& \bigcap_{\mu \in I_{n}^{*}}\left\{\xi \in \mathfrak{T}_{\mathfrak{g}}:\left(\forall \nu \in I_{\mu}^{*}\right)\left(\xi \in \mathcal{S}_{\mathfrak{g}, \nu} \in \mathfrak{g}-\mathrm{K}\left[\mathfrak{T}_{\mathfrak{g}}\right]\right)\right\} \subseteq \mathfrak{g}-\mathrm{K}\left[\mathfrak{T}_{\mathfrak{g}}\right]
\end{aligned}
$$

Proof. Let $\left\{\mathcal{R}_{\mathfrak{g}, \nu} \in \mathfrak{g}-\mathrm{O}\left[\mathfrak{T}_{\mathfrak{g}}\right]: \nu \in I_{n}^{*}\right\}$ and $\left\{\mathcal{S}_{\mathfrak{g}, \nu} \in \mathfrak{g}-\mathrm{K}\left[\mathfrak{T}_{\mathfrak{g}}\right]: \nu \in I_{n}^{*}\right\}$, respectively, be finite collections of $\mathfrak{g}-\mathfrak{T}_{\mathfrak{g}}$-open and $\mathfrak{g}$ - $\mathfrak{T}_{\mathfrak{g}}$-closed sets in a $\mathcal{T}_{\mathfrak{g}}$-space $\mathfrak{T}_{\mathfrak{g}}$. Then, since $\left(\mathcal{R}_{\mathfrak{g}, \nu}, \mathcal{S}_{\mathfrak{g}, \nu}\right) \in \mathfrak{g}$-O $\left[\mathfrak{T}_{\mathfrak{g}}\right] \times \mathfrak{g}-\mathrm{K}\left[\mathfrak{T}_{\mathfrak{g}}\right]$, there exists $\left(\mathcal{O}_{\mathfrak{g}, \nu}, \mathcal{K}_{\mathfrak{g}, \nu}\right) \in \mathcal{T}_{\mathfrak{g}} \times \neg \mathcal{T}_{\mathfrak{g}}$ such that the propositional formulas $\mathrm{P}_{\mathfrak{g}}\left(\mathcal{R}_{\mathfrak{g}, \nu}, \mathcal{O}_{\mathfrak{g}, \nu} ; \mathbf{o p}_{\mathfrak{g}}(\cdot) ; \subseteq\right)$ and $\mathrm{P}_{\mathfrak{g}}\left(\mathcal{S}_{\mathfrak{g}, \nu}, \mathcal{K}_{\mathfrak{g}, \nu} ; \mathbf{o p}_{\mathfrak{g}}(\cdot) ; \supseteq\right)$ hold true for every index $\nu \in I_{n}^{*}$. Consequently, the propositional formulas $\bigvee_{\nu \in I_{n}^{*}} \mathrm{P}_{\mathfrak{g}}\left(\mathcal{R}_{\mathfrak{g}, \nu}, \mathcal{O}_{\mathfrak{g}, \nu} ; \mathbf{o p}_{\mathfrak{g}}(\cdot) ; \subseteq\right)$ and $\bigwedge_{\nu \in I_{n}^{*}} \mathrm{P}_{\mathfrak{g}}\left(\mathcal{S}_{\mathfrak{g}, \nu}, \mathcal{K}_{\mathfrak{g}, \nu} ; \mathbf{o p}_{\mathfrak{g}}(\cdot) ; \supseteq\right)$ also hold true. Since

$$
\begin{aligned}
\bigcup_{\mu \in I_{n}^{*}} \mathcal{R}_{\mathfrak{g}, \nu} & \longleftrightarrow \bigcup_{\mu \in I_{n}^{*}}\left\{\mathcal{R}_{\mathfrak{g}, \nu} \in \mathfrak{g}-\mathrm{O}\left[\mathfrak{T}_{\mathfrak{g}}\right]: \nu \in I_{\mu}^{*}\right\} \\
& \longleftrightarrow \bigcup_{\mu \in I_{n}^{*}}\left\{\xi \in \mathfrak{T}_{\mathfrak{g}}:\left(\exists \nu \in I_{\mu}^{*}\right)\left(\xi \in \mathcal{S}_{\mathfrak{g}, \nu} \in \mathfrak{g}-\mathrm{O}\left[\mathfrak{T}_{\mathfrak{g}}\right]\right)\right\} \subseteq \bigcup_{\nu \in I_{n}^{*}} \operatorname{op}_{\mathfrak{g}}\left(\mathcal{O}_{\mathfrak{g}, \nu}\right) \\
\bigcup_{\nu \in I_{n}^{*}} \mathrm{op}_{\mathfrak{g}}\left(\mathcal{O}_{\mathfrak{g}, \nu}\right) & \longleftrightarrow \bigcap_{\mu \in I_{n}^{*}}\left\{\mathcal{S}_{\mathfrak{g}, \nu} \in \mathfrak{g}-\mathrm{K}\left[\mathfrak{T}_{\mathfrak{g}}\right]: \nu \in I_{\mu}^{*}\right\} \\
& \longleftrightarrow \bigcap_{\mu \in I_{n}^{*}}\left\{\xi \in \mathfrak{T}_{\mathfrak{g}}:\left(\forall \nu \in I_{\mu}^{*}\right)\left(\xi \in \mathcal{S}_{\mathfrak{g}, \nu} \in \mathfrak{g}-\mathrm{K}\left[\mathfrak{T}_{\mathfrak{g}}\right]\right)\right\} \supseteq \bigcap_{\nu \in I_{n}^{*}} \neg \mathrm{op}_{\mathfrak{g}}\left(\mathcal{K}_{\mathfrak{g}, \nu}\right)
\end{aligned}
$$

it results that $\bigcup_{\mu \in I_{n}^{*}} \mathcal{R}_{\mathfrak{g}, \nu} \subseteq \bigcup_{\nu \in I_{n}^{*}} \mathrm{op}_{\mathfrak{g}}\left(\mathcal{O}_{\mathfrak{g}, \nu}\right)$ and $\bigcap_{\mu \in I_{n}^{*}} \mathcal{S}_{\mathfrak{g}, \nu} \supseteq \bigcap_{\nu \in I_{n}^{*}} \neg \mathrm{op}_{\mathfrak{g}}\left(\mathcal{K}_{\mathfrak{g}, \nu}\right)$. But it holds that $\bigcup_{\mu \in I_{n}^{*}} \mathcal{R}_{\mathfrak{g}, \nu} \subseteq \operatorname{op}_{\mathfrak{g}}\left(\bigcup_{\mu \in I_{n}^{*}} \mathcal{R}_{\mathfrak{g}, \nu}\right) \in \mathfrak{g}-\mathrm{O}\left[\mathfrak{T}_{\mathfrak{g}}\right]$ and $\bigcap_{\mu \in I_{n}^{*}} \mathcal{S}_{\mathfrak{g}, \nu} \supseteq \neg \mathrm{op}_{\mathfrak{g}}\left(\bigcap_{\mu \in I_{n}^{*}} \mathcal{S}_{\mathfrak{g}, \nu}\right) \in \mathfrak{g}-\mathrm{K}\left[\mathfrak{T}_{\mathfrak{g}}\right]$. Consequently, there exists $\left(\mathcal{O}_{\mathfrak{g}}, \mathcal{K}_{\mathfrak{g}}\right) \in \mathcal{T}_{\mathfrak{g}} \times \neg \mathcal{T}_{\mathfrak{g}}$ such that $\bigcup_{\mu \in I_{n}^{*}} \mathcal{R}_{\mathfrak{g}, \nu} \subseteq \operatorname{op}_{\mathfrak{g}}\left(\mathcal{O}_{\mathfrak{g}}\right) \in \mathfrak{g}-\mathrm{O}\left[\mathfrak{T}_{\mathfrak{g}}\right]$ and $\bigcap_{\mu \in I_{n}^{*}} \mathcal{S}_{\mathfrak{g}, \nu} \supseteq \neg \mathrm{op}_{\mathfrak{g}}\left(\mathcal{K}_{\mathfrak{g}}\right) \in \mathfrak{g}-\mathrm{K}\left[\mathfrak{T}_{\mathfrak{g}}\right]$, implying that both $\mathrm{P}_{\mathfrak{g}}\left(\bigcup_{\nu \in I_{n}^{*}} \mathcal{R}_{\mathfrak{g}, \nu}, \bigcup_{\nu \in I_{\mu}^{*}} \mathcal{O}_{\mathfrak{g}, \nu} ; \mathbf{o p}_{\mathfrak{g}}(\cdot) ; \subseteq\right)$ and $\mathrm{P}_{\mathfrak{g}}\left(\bigcup_{\nu \in I_{n}^{*}} \mathcal{S}_{\mathfrak{g}, \nu}, \bigcup_{\nu \in I_{\mu}^{*}} \mathcal{K}_{\mathfrak{g}, \nu} ; \mathbf{o p}_{\mathfrak{g}}(\cdot) ; \supseteq\right)$, respectively, hold true. But,

$$
\begin{aligned}
\mathrm{P}_{\mathfrak{g}}\left(\bigcup_{\nu \in I_{n}^{*}} \mathcal{R}_{\mathfrak{g}, \nu}, \mathcal{O}_{\mathfrak{g}} ; \mathbf{o p}_{\mathfrak{g}}(\cdot) ; \subseteq\right) & =\bigvee_{\nu \in I_{n}^{*}} \mathrm{P}_{\mathfrak{g}}\left(\mathcal{R}_{\mathfrak{g}, \nu}, \mathcal{O}_{\mathfrak{g}} ; \mathbf{o p}_{\mathfrak{g}}(\cdot) ; \subseteq\right) \\
\mathrm{P}_{\mathfrak{g}}\left(\bigcup_{\nu \in I_{n}^{*}} \mathcal{S}_{\mathfrak{g}, \nu}, \mathcal{K}_{\mathfrak{g}} ; \mathbf{o p}_{\mathfrak{g}}(\cdot) ; \supseteq\right) & =\bigwedge_{\nu \in I_{n}^{*}} \mathrm{P}_{\mathfrak{g}}\left(\mathcal{S}_{\mathfrak{g}, \nu}, \mathcal{K}_{\mathfrak{g}} ; \mathbf{o p}_{\mathfrak{g}}(\cdot) ; \supseteq\right)
\end{aligned}
$$


Hence, it suffices to set

$$
\begin{aligned}
\mathrm{P}_{\mathfrak{g}}\left(\mathcal{R}_{\mathfrak{g}}, \mathcal{O}_{\mathfrak{g}} ; \mathbf{o p}_{\mathfrak{g}}(\cdot) ; \subseteq\right)=\bigvee_{\nu \in I_{n}^{*}} \mathrm{P}_{\mathfrak{g}}\left(\mathcal{R}_{\mathfrak{g}, \nu}, \mathcal{O}_{\mathfrak{g}} ; \mathbf{o p}_{\mathfrak{g}}(\cdot) ; \subseteq\right) \\
\mathrm{P}_{\mathfrak{g}}\left(\mathcal{S}_{\mathfrak{g}}, \mathcal{K}_{\mathfrak{g}} ; \mathbf{o p}_{\mathfrak{g}}(\cdot) ; \supseteq\right)=\bigvee_{\nu \in I_{n}^{*}} \mathrm{P}_{\mathfrak{g}}\left(\mathcal{S}_{\mathfrak{g}, \nu}, \mathcal{K}_{\mathfrak{g}} ; \mathbf{o p}_{\mathfrak{g}}(\cdot) ; \supseteq\right)
\end{aligned}
$$

and the theorem is proved.

If in $\mathrm{P}_{\mathfrak{g}}\left(\mathcal{S}_{\mathfrak{g}}, \mathcal{O}_{\mathfrak{g}}, \mathcal{K}_{\mathfrak{g}} ; \mathbf{o p}_{\mathfrak{g}}(\cdot) ; \subseteq, \supseteq\right)$ it be assumed that $\left(\mathcal{O}_{\mathfrak{g}}, \mathcal{K}_{\mathfrak{g}}\right) \in \mathfrak{g}-\mathrm{S}\left[\mathfrak{T}_{\mathfrak{g}}\right] \times \mathfrak{g}-\mathrm{S}\left[\mathfrak{T}_{\mathfrak{g}}\right]$, we have the following theorem:

Theorem 3.20. Let $\left(\mathcal{S}_{\mathfrak{g}}, \mathcal{O}_{\mathfrak{g}}, \mathcal{K}_{\mathfrak{g}}\right) \in \mathfrak{T}_{\mathfrak{g}} \times \mathcal{T}_{\mathfrak{g}} \times \neg \mathcal{T}_{\mathfrak{g}}$ in a $\mathcal{T}_{\mathfrak{g}}$-space $\mathfrak{T}_{\mathfrak{g}}$. If $\left(\mathcal{O}_{\mathfrak{g}}, \mathcal{K}_{\mathfrak{g}}\right) \in \mathfrak{g}-\mathrm{O}\left[\mathfrak{T}_{\mathfrak{g}}\right] \times \mathfrak{g}-\mathrm{K}\left[\mathfrak{T}_{\mathfrak{g}}\right]$, then

$$
\left\{\mathcal{S}_{\mathfrak{g}} \subset \mathfrak{T}_{\mathfrak{g}}: \mathrm{P}_{\mathfrak{g}}\left(\mathcal{S}_{\mathfrak{g}}, \mathcal{O}_{\mathfrak{g}}, \mathcal{K}_{\mathfrak{g}} ; \mathbf{o p}_{\mathfrak{g}}(\cdot) ; \subseteq, \supseteq\right)\right\} \subseteq \mathfrak{g}-\mathrm{S}\left[\mathfrak{T}_{\mathfrak{g}}\right]
$$

Proof. It is clear that

$$
\mathrm{P}_{\mathfrak{g}}\left(\mathcal{S}_{\mathfrak{g}}, \mathcal{O}_{\mathfrak{g}}, \mathcal{K}_{\mathfrak{g}} ; \mathbf{o p}_{\mathfrak{g}}(\cdot) ; \subseteq, \supseteq\right)=\mathrm{P}_{\mathfrak{g}}\left(\mathcal{S}_{\mathfrak{g}}, \mathcal{O}_{\mathfrak{g}} ; \mathbf{o p}_{\mathfrak{g}}(\cdot) ; \subseteq\right) \vee \mathrm{P}_{\mathfrak{g}}\left(\mathcal{S}_{\mathfrak{g}}, \mathcal{K}_{\mathfrak{g}} ; \mathbf{o p}_{\mathfrak{g}}(\cdot) ; \supseteq\right)
$$

and the Boolean-valued functions surrounding $\vee$ hold on $\mathfrak{T}_{\mathfrak{g}} \times \mathcal{T}_{\mathfrak{g}} \cup \neg \mathcal{T}_{\mathfrak{g}} \times \mathcal{L}_{\mathfrak{g}}[\Omega] \times\{\subseteq, \supseteq\}$. Consequently, the following two cases must be considered in proving the theorem:

CASE I. Let $\mathrm{P}_{\mathfrak{g}}\left(\mathcal{S}_{\mathfrak{g}}, \mathcal{O}_{\mathfrak{g}} ; \mathbf{o p}_{\mathfrak{g}}(\cdot) ; \subseteq\right)$ hold on $\mathfrak{T}_{\mathfrak{g}} \times \mathcal{T}_{\mathfrak{g}} \cup \neg \mathcal{T}_{\mathfrak{g}} \times \mathcal{L}_{\mathfrak{g}}[\Omega] \times\{\subseteq, \supseteq\}$. Then, $\mathcal{S}_{\mathfrak{g}} \subseteq$ op $_{\mathfrak{g}}\left(\mathcal{O}_{\mathfrak{g}}\right)$. But, $\mathcal{O}_{\mathfrak{g}} \in \mathfrak{g}-\mathrm{O}\left[\mathfrak{T}_{\mathfrak{g}}\right]$, and consequently, it follows that $\mathcal{O}_{\mathfrak{g}} \subseteq \operatorname{op}_{\mathfrak{g}}\left(\mathcal{O}_{\mathfrak{g}, \nu}\right)$ and $\operatorname{op}_{\mathfrak{g}}\left(\mathcal{O}_{\mathfrak{g}}\right) \subseteq \operatorname{op}_{\mathfrak{g}} \circ \mathrm{op}_{\mathfrak{g}}\left(\mathcal{O}_{\mathfrak{g}, \nu}\right) \subseteq$ $\operatorname{op}_{\mathfrak{g}}\left(\mathcal{O}_{\mathfrak{g}, \nu}\right)$ for some $\mathcal{O}_{\mathfrak{g}, \nu} \in \mathcal{T}_{\mathfrak{g}}$, by the properties of the $\mathfrak{g}$-operator. Hence, $\mathrm{P}_{\mathfrak{g}}\left(\mathcal{S}_{\mathfrak{g}}, \mathcal{O}_{\mathfrak{g}, \nu} ; \mathrm{op}_{\mathfrak{g}}(\cdot) ; \subseteq\right)$ holds on $\mathfrak{T}_{\mathfrak{g}} \times \mathcal{T}_{\mathfrak{g}} \cup \neg \mathcal{T}_{\mathfrak{g}} \times \mathcal{L}_{\mathfrak{g}}[\Omega] \times\{\subseteq, \supseteq\}$.

CASE II. Let $\mathrm{P}_{\mathfrak{g}}\left(\mathcal{S}_{\mathfrak{g}}, \mathcal{K}_{\mathfrak{g}} ; \mathbf{o p}_{\mathfrak{g}}(\cdot) ; \supseteq\right)$ hold on $\mathfrak{T}_{\mathfrak{g}} \times \mathcal{T}_{\mathfrak{g}} \cup \neg \mathcal{T}_{\mathfrak{g}} \times \mathcal{L}_{\mathfrak{g}}[\Omega] \times\{\subseteq, \supseteq\}$. Then, $\mathcal{S}_{\mathfrak{g}} \supseteq \neg \operatorname{op}_{\mathfrak{g}}\left(\mathcal{K}_{\mathfrak{g}}\right)$. But, $\mathcal{K}_{\mathfrak{g}} \in \mathfrak{g}-\mathrm{K}\left[\mathfrak{T}_{\mathfrak{g}}\right]$, and consequently, it follows that $\mathcal{K}_{\mathfrak{g}} \supseteq \neg \mathrm{op}_{\mathfrak{g}}\left(\mathcal{K}_{\mathfrak{g}, \nu}\right)$ and $\mathrm{op}_{\mathfrak{g}}\left(\mathcal{K}_{\mathfrak{g}}\right) \supseteq \neg \mathrm{op}_{\mathfrak{g}} \circ \neg \mathrm{op}_{\mathfrak{g}}\left(\mathcal{K}_{\mathfrak{g}, \nu}\right) \supseteq$ $\neg \operatorname{op}_{\mathfrak{g}}\left(\mathcal{K}_{\mathfrak{g}, \nu}\right)$ for some $\mathcal{K}_{\mathfrak{g}, \nu} \in \neg \mathcal{T}_{\mathfrak{g}}$, by the properties of the $\mathfrak{g}$-operator. Hence, $\mathrm{P}_{\mathfrak{g}}\left(\mathcal{S}_{\mathfrak{g}}, \mathcal{K}_{\mathfrak{g}, \nu} ; \mathrm{op}_{\mathfrak{g}}(\cdot) ; \supseteq\right)$ holds on $\mathfrak{T}_{\mathfrak{g}} \times \mathcal{T}_{\mathfrak{g}} \cup \neg \mathcal{T}_{\mathfrak{g}} \times \mathcal{L}_{\mathfrak{g}}[\Omega] \times\{\subseteq, \supseteq\}$.

From CASE I. and CASE II., it follows that

$$
\begin{aligned}
& \left\{\mathcal{S}_{\mathfrak{g}} \subset \mathfrak{T}_{\mathfrak{g}}: \mathrm{P}_{\mathfrak{g}}\left(\mathcal{S}_{\mathfrak{g}}, \mathcal{O}_{\mathfrak{g}} ; \mathbf{o p}_{\mathfrak{g}}(\cdot) ; \subseteq\right)\right\} \subseteq \mathfrak{g}-\mathrm{O}\left[\mathfrak{T}_{\mathfrak{g}}\right] \\
& \left\{\mathcal{S}_{\mathfrak{g}} \subset \mathfrak{T}_{\mathfrak{g}}: \mathrm{P}_{\mathfrak{g}}\left(\mathcal{S}_{\mathfrak{g}}, \mathcal{K}_{\mathfrak{g}} ; \mathbf{o p}_{\mathfrak{g}}(\cdot) ; \supseteq\right)\right\} \subseteq \mathfrak{g}-\mathrm{K}\left[\mathfrak{T}_{\mathfrak{g}}\right]
\end{aligned}
$$

But, since $\mathfrak{g}-\mathrm{S}\left[\mathfrak{T}_{\mathfrak{g}}\right]=\mathfrak{g}-\mathrm{O}\left[\mathfrak{T}_{\mathfrak{g}}\right] \cup \mathfrak{g}-\mathrm{K}\left[\mathfrak{T}_{\mathfrak{g}}\right]$, the proof of the theorem at once follows.

The following theorem shows that the class $\mathfrak{g}-\mathrm{S}\left[\mathfrak{T}_{\mathfrak{g}}\right]$, upon satisfaction of two conditions, is the smallest class of $\mathfrak{g}$ - $\mathfrak{T}_{\mathfrak{g}}$-sets in the $\mathcal{T}_{\mathfrak{g}}$-space $\mathfrak{T}_{\mathfrak{g}}$.

Theorem 3.21. Let $\mathfrak{g}-\mathrm{S}_{0}\left[\mathfrak{T}_{\mathfrak{g}}\right]=\mathfrak{g}-\mathrm{O}_{0}\left[\mathfrak{T}_{\mathfrak{g}}\right] \cup \mathfrak{g}-\mathrm{K}_{0}\left[\mathfrak{T}_{\mathfrak{g}}\right]$ be a class of $\mathfrak{g}$ - $\mathfrak{T}_{\mathfrak{g}}$-sets in a $\mathcal{T}_{\mathfrak{g}}$-space $\mathfrak{T}_{\mathfrak{g}}$ such that the following two conditions are satisfied:

$i$. If $\left(\mathcal{O}_{\mathfrak{g}}, \mathcal{K}_{\mathfrak{g}}\right) \in \mathfrak{g}-\mathrm{O}_{0}\left[\mathfrak{T}_{\mathfrak{g}}\right] \times \mathfrak{g}-\mathrm{K}_{0}\left[\mathfrak{T}_{\mathfrak{g}}\right]$ and $\mathrm{P}_{\mathfrak{g}}\left(\mathcal{S}_{\mathfrak{g}}, \mathcal{O}_{\mathfrak{g}}, \mathcal{K}_{\mathfrak{g}} ; \mathbf{o p}_{\mathfrak{g}}(\cdot) ; \subseteq, \supseteq\right)$ holds on $\mathfrak{T}_{\mathfrak{g}} \times \mathfrak{g}-\mathrm{O}_{0}\left[\mathfrak{T}_{\mathfrak{g}}\right] \times$ $\mathfrak{g}-\mathrm{K}_{0}\left[\mathfrak{T}_{\mathfrak{g}}\right] \times \mathcal{L}_{\mathfrak{g}}[\Omega] \times\{\subseteq, \supseteq\}$, then $\mathcal{S}_{\mathfrak{g}} \in \mathfrak{g}-\mathrm{S}_{0}\left[\mathfrak{T}_{\mathfrak{g}}\right]$.

ii. The relation $\mathcal{S}_{\mathfrak{g}} \in \mathrm{S}\left[\mathfrak{T}_{\mathfrak{g}}\right]$ implies $\mathcal{S}_{\mathfrak{g}} \in \mathfrak{g}-\mathrm{S}_{0}\left[\mathfrak{T}_{\mathfrak{g}}\right]$.

Then, $\mathfrak{g}-\mathrm{S}\left[\mathfrak{T}_{\mathfrak{g}}\right] \subseteq \mathfrak{g}-\mathrm{S}_{0}\left[\mathfrak{T}_{\mathfrak{g}}\right]$.

Proof. Let $\mathcal{S}_{\mathfrak{g}} \in \mathfrak{g}-\mathrm{S}\left[\mathfrak{T}_{\mathfrak{g}}\right]$. Then, $\mathrm{P}_{\mathfrak{g}}\left(\mathcal{S}_{\mathfrak{g}}, \mathcal{O}_{\mathfrak{g}}, \mathcal{K}_{\mathfrak{g}} ; \mathbf{o p}_{\mathfrak{g}}(\cdot) ; \subseteq, \supseteq\right)$ holds on $\mathfrak{T}_{\mathfrak{g}} \times \mathrm{O}\left[\mathfrak{T}_{\mathfrak{g}}\right] \times \mathrm{K}\left[\mathfrak{T}_{\mathfrak{g}}\right] \times \mathcal{L}_{\mathfrak{g}}[\Omega] \times$ $\{\subseteq, \supseteq\}$ for some pair $\left(\mathcal{O}_{\mathfrak{g}}, \mathcal{K}_{\mathfrak{g}}\right) \in \mathrm{O}\left[\mathfrak{T}_{\mathfrak{g}}\right] \times \mathrm{K}\left[\mathfrak{T}_{\mathfrak{g}}\right]$. But, $\left(\mathcal{O}_{\mathfrak{g}}, \mathcal{K}_{\mathfrak{g}}\right) \in \mathrm{O}\left[\mathfrak{T}_{\mathfrak{g}}\right] \times \mathrm{K}\left[\mathfrak{T}_{\mathfrak{g}}\right]$ implies $\left(\mathcal{O}_{\mathfrak{g}}, \mathcal{K}_{\mathfrak{g}}\right) \in$ $\mathfrak{g}-\mathrm{O}_{0}\left[\mathfrak{T}_{\mathfrak{g}}\right] \times \mathfrak{g}-\mathrm{K}_{0}\left[\mathfrak{T}_{\mathfrak{g}}\right]$ by $(i$.$) , and the latter together with the trueness of \mathrm{P}_{\mathfrak{g}}\left(\mathcal{S}_{\mathfrak{g}}, \mathcal{O}_{\mathfrak{g}}, \mathcal{K}_{\mathfrak{g}} ; \mathbf{o p}_{\mathfrak{g}}(\cdot) ; \subseteq, \supseteq\right)$ on $\mathfrak{T}_{\mathfrak{g}} \times \mathfrak{g}-\mathrm{O}_{0}\left[\mathfrak{T}_{\mathfrak{g}}\right] \times \mathfrak{g}-\mathrm{K}_{0}\left[\mathfrak{T}_{\mathfrak{g}}\right] \times \mathcal{L}_{\mathfrak{g}}[\Omega] \times\{\subseteq, \supseteq\}$ implies $\mathcal{S}_{\mathfrak{g}} \in \mathfrak{g}-\mathrm{S}_{0}\left[\mathfrak{T}_{\mathfrak{g}}\right]$ by $\left(i i\right.$.). Thus, $\mathfrak{g}-\mathrm{S}\left[\mathfrak{T}_{\mathfrak{g}}\right] \subseteq \mathfrak{g}-\mathrm{S}_{0}\left[\mathfrak{T}_{\mathfrak{g}}\right]$, which completes the proof. 
In the earlier discussion, the set $\Omega \subset \mathfrak{U}$ carried the $\mathfrak{g}$-topology $\mathcal{T}_{\mathfrak{g}}(\Omega)$. A $\mathfrak{g}$-topology of this kind will be termed an absolute $\mathfrak{g}$-topology. To this end, if $\Gamma \subseteq \Omega$ is any subset of $\Omega$ then, obviously, we would expect $\Gamma$ to carry the $\mathfrak{g}$-topology $\mathcal{T}_{\mathfrak{g}}(\Gamma)$. But, since $\mathcal{T}_{\mathfrak{g}}(\Gamma) \subseteq \mathcal{T}_{\mathfrak{g}}(\Omega)$, as a consequence of the fact that $\mathcal{T}_{\mathfrak{g}}: \mathcal{P}(\Gamma) \longrightarrow \mathcal{P}(\Gamma)$ is the one-valued restriction map of $\mathcal{T}_{\mathfrak{g}}: \mathcal{P}(\Omega) \longrightarrow \mathcal{P}(\Omega)$, which follows from the statement, $\Gamma \subseteq \Omega$ implies $\mathcal{P}(\Gamma) \subseteq \mathcal{P}(\Omega)$, it does make sense to term $\mathcal{T}_{\mathfrak{g}}(\Gamma)$ a relative $\mathfrak{g}$-topology. In order to determine what any $\mathfrak{g}$-set-theoretic concepts for the $\mathcal{T}_{\mathfrak{g}}$-space $\mathfrak{T}_{\mathfrak{g}}=\left(\Omega, \mathcal{T}_{\mathfrak{g}}(\Omega)\right)$ becomes when discussion is restricted to $\Gamma \subseteq \Omega$, it merely suffices to regard $\Gamma$ as the set which carries the relative $\mathfrak{g}$-topology $\mathcal{T}_{\mathfrak{g}}(\Gamma)$ and carry over the discussion verbatim.

Definition 3.22 $\left(\mathcal{T}_{\mathfrak{g}}\right.$-Subspace). Let $\mathfrak{T}_{\mathfrak{g}}(\Omega):=\left(\Omega, \mathcal{T}_{\mathfrak{g}}(\Omega)\right)$ be a $\mathcal{T}_{\mathfrak{g}}$-space $\mathfrak{T}_{\mathfrak{g}}$, where $\Omega \subset \mathfrak{U}$ carries the absolute $\mathfrak{g}$-topology $\mathcal{T}_{\mathfrak{g}}: \mathcal{P}(\Omega) \longrightarrow \mathcal{P}(\Omega)$, and let $\mathcal{P}(\Gamma):=\left\{\mathcal{O}_{\mathfrak{g}, \nu}: \mathcal{O}_{\mathfrak{g}, \nu} \subset \Gamma\right\}$ be the family of all subsets $\mathcal{O}_{\mathfrak{g}, 1}, \mathcal{O}_{\mathfrak{g}, 2}, \ldots$, of any subset $\Gamma \subseteq \Omega$ of $\Omega$, then every one-valued restriction map of the type

$$
\mathcal{T}_{\mathfrak{g}}: \mathcal{P}(\Gamma) \longmapsto \mathcal{T}_{\mathfrak{g}}(\Gamma):=\left\{\mathcal{O}_{\mathfrak{g}} \cap \Gamma: \mathcal{O}_{\mathfrak{g}} \in \mathcal{T}_{\mathfrak{g}}(\Omega)\right\}
$$

defines a "relative $\mathfrak{g}$-topology on $\Gamma$," and the structure $\mathfrak{T}_{\mathfrak{g}}(\Gamma):=\left(\Gamma, \mathcal{T}_{\mathfrak{g}}(\Gamma)\right)$ is called a " $\mathcal{T}_{\mathfrak{g}}$-subspace."

Theorem 3.23. Let $\mathcal{S}_{\mathfrak{g}} \subset \mathfrak{T}_{\mathfrak{g}}(\Gamma) \subseteq \mathfrak{T}_{\mathfrak{g}}(\Omega)$, where $\mathfrak{T}_{\mathfrak{g}}(\Gamma)=\left(\Gamma, \mathcal{T}_{\mathfrak{g}}(\Gamma)\right)$ is the $\mathcal{T}_{\mathfrak{g}}$-subspace of a $\mathcal{T}_{\mathfrak{g}}$-space $\mathfrak{T}_{\mathfrak{g}}(\Omega)=\left(\Omega, \mathcal{T}_{\mathfrak{g}}(\Omega)\right)$. If $\mathcal{S}_{\mathfrak{g}} \in \mathfrak{g}-\mathrm{S}\left[\mathfrak{T}_{\mathfrak{g}}(\Omega)\right]$, then $\mathcal{S}_{\mathfrak{g}} \in \mathfrak{g}-\mathrm{S}\left[\mathfrak{T}_{\mathfrak{g}}(\Gamma)\right]$.

Proof. If $\mathcal{S}_{\mathfrak{g}} \in \mathfrak{g}-\mathrm{S}\left[\mathfrak{T}_{\mathfrak{g}}(\Omega)\right]$, then $\mathrm{P}_{\mathfrak{g}}\left(\mathcal{S}_{\mathfrak{g}}, \mathcal{O}_{\mathfrak{g}}, \mathcal{K}_{\mathfrak{g}} ; \mathbf{o p}_{\mathfrak{g}}(\cdot) ; \subseteq, \supseteq\right)$ holds on $\mathfrak{T}_{\mathfrak{g}}(\Omega) \times \mathcal{T}_{\mathfrak{g}}(\Omega) \cup \neg \mathcal{T}_{\mathfrak{g}}(\Omega) \times$ $\mathcal{L}_{\mathfrak{g}}[\Omega] \times\{\subseteq, \supseteq\}$. Therefore, if $\mathcal{S}_{\mathfrak{g}} \in \mathfrak{g}-\mathrm{S}\left[\mathfrak{T}_{\mathfrak{g}}(\Gamma)\right]$, then $\mathrm{P}_{\mathfrak{g}}\left(\mathcal{S}_{\mathfrak{g}} \cap \Gamma, \mathcal{O}_{\mathfrak{g}} \cap \Gamma, \mathcal{K}_{\mathfrak{g}} \cap \Gamma ; \mathbf{o p}_{\mathfrak{g}}(\cdot) ; \subseteq, \supseteq\right)$ holds on $\mathfrak{T}_{\mathfrak{g}}(\Gamma) \times \mathcal{T}_{\mathfrak{g}}(\Gamma) \cup \neg \mathcal{T}_{\mathfrak{g}}(\Gamma) \times \mathcal{L}_{\mathfrak{g}}[\Gamma] \times\{\subseteq, \supseteq\}$. But, since $\mathcal{S}_{\mathfrak{g}} \cap \Gamma=\mathcal{S}_{\mathfrak{g}} \in \mathfrak{g}-\mathrm{S}\left[\mathfrak{T}_{\mathfrak{g}}(\Gamma)\right], \mathcal{O}_{\mathfrak{g}} \cap \Gamma=\mathcal{O}_{\mathfrak{g}} \in \mathcal{T}_{\mathfrak{g}}(\Gamma)$, and $\mathcal{K}_{\mathfrak{g}} \cap \Gamma=\mathcal{K}_{\mathfrak{g}} \in \mathcal{T}_{\mathfrak{g}}(\Gamma)$, it follows that $\mathcal{S}_{\mathfrak{g}} \in \mathfrak{g}-\mathrm{S}\left[\mathfrak{T}_{\mathfrak{g}}(\Gamma)\right]$ whenever $\mathcal{S}_{\mathfrak{g}} \in \mathfrak{g}-\mathrm{S}\left[\mathfrak{T}_{\mathfrak{g}}(\Omega)\right]$, and the theorem is proved.

Definition 3.24 (Cartesian Product). The Cartesian product of an arbitrary family $\left\{\Omega_{\nu} \subset \mathfrak{U}: \nu \in\right.$ $\left.I_{n}^{*}\right\}$ of sets is the set of functions $\phi: I_{n}^{*} \longrightarrow \bigcup_{\nu \in I_{n}^{*}} \Omega_{\nu}$ such that $\phi: \nu \longmapsto \Omega_{\nu}$ for every $\nu \in I_{n}^{*}$. It is denoted by $\bigotimes_{\nu \in I_{n}^{*}} \Omega_{\nu}$ and satisfies the following properties:

i. $\bigotimes_{\nu=\mu} \Omega_{\nu}=\Omega_{\mu} \quad \forall \mu \in I_{n}^{*}$

ii. $\bigotimes_{\nu \in I_{\mu+1}^{*}} \Omega_{\nu}=\left(\bigotimes_{\nu \in I_{\mu}^{*}} \Omega_{\nu}\right) \times \Omega_{\mu+1} \quad \forall \mu \in I_{n-1}^{*}$

The projection map which gives the projection of the Cartesian product set $\bigotimes_{\nu \in I_{n}^{*}} \Omega_{\nu}$ onto the $\mu^{\text {th }}$ factor of $\bigotimes_{\nu \in I_{n}^{*}} \Omega_{\nu}$ is defined as thus.

Definition 3.25 (Projection). Let $\left\{\Omega_{\nu} \subset \mathfrak{U}: \nu \in I_{n}^{*}\right\}$ be any class of sets and let $\bigotimes_{\nu \in I_{n}^{*}} \Omega_{\nu}$ denotes the Cartesian product of these sets. The map

$$
\operatorname{proj}_{\mu}: \bigotimes_{\nu \in I_{n}^{*}} \Omega_{\nu} \longrightarrow \Omega_{\mu} \quad\left(\operatorname{proj}_{\mu}\left(\bigotimes_{\nu \in I_{n}^{*}} \Omega_{\nu}\right)=\Omega_{\mu}\right)
$$

is called the projection of the Cartesian product set $\bigotimes_{\nu \in I_{n}^{*}} \Omega_{\nu}$ onto the $\mu^{\text {th }}$ factor of $\bigotimes_{\nu \in I_{n}^{*}} \Omega_{\nu}$.

To generate all $\mathcal{T}_{\mathfrak{g}}$-open sets in a $\mathcal{T}_{\mathfrak{g}}$-space $\mathfrak{T}_{\mathfrak{g}}$, a basis $\mathcal{B}\left[\mathcal{T}_{\mathfrak{g}}\right]$ for $\mathfrak{T}_{\mathfrak{g}}$ must be supplied, and the following definition is worth considering.

Definition 3.26 $\left(\mathcal{T}_{\mathfrak{g}}\right.$-Basis). A subclass $\mathcal{B}\left[\mathcal{T}_{\mathfrak{g}}\left(\Omega_{\mu}\right)\right] \subseteq \mathcal{T}_{\mathfrak{g}}\left(\Omega_{\mu}\right)$ consisting of $\mathcal{T}_{\mathfrak{g}}$-open sets in a $\mathcal{T}_{\mathfrak{g}}$-space $\mathfrak{T}_{\mathfrak{g}}\left(\Omega_{\mu}\right):=\left(\Omega_{\mu}, \mathcal{T}_{\mathfrak{g}}\left(\Omega_{\mu}\right)\right)$, defined by

$$
\mathcal{B}\left[\mathcal{T}_{\mathfrak{g}}\left(\Omega_{\mu}\right)\right]:=\left\{\mathcal{O}_{\mathfrak{g}, \sigma(\nu, \mu)}:(\nu, \mu, \sigma(\nu, \mu)) \in I_{\infty}^{*} \times\{\mu\} \times I_{\infty}^{*}\right\}
$$

is said to be a base for $\mathcal{T}_{\mathfrak{g}}: \mathcal{P}\left(\Omega_{\mu}\right) \longrightarrow \mathcal{P}\left(\Omega_{\mu}\right)$ if and only if

$$
\forall\left(\mu, \sigma(\mu), \mathcal{O}_{\mathfrak{g}, \sigma(\mu)}\right) \in\{\mu\} \times I_{\infty}^{*} \times \mathcal{T}_{\mathfrak{g}}\left(\Omega_{\mu}\right), \exists I_{\sigma(\mu)} \subseteq I_{\infty}^{*}: \mathcal{O}_{\mathfrak{g}, \sigma(\mu)}=\bigcup_{\nu \in I_{\sigma(\mu)}^{*}} \mathcal{O}_{\mathfrak{g}, \sigma(\nu, \mu)}
$$


With regards to the terminology employed, $\mathcal{B}\left[\mathcal{T}_{\mathfrak{g}}\left(\Omega_{\mu}\right)\right]$ is called a $\mathcal{T}_{\mathfrak{g}}$-basis and its elements, $\mathcal{B}_{\mathcal{T}_{\mathfrak{g}}}$ open sets, because they are $\mathcal{T}_{\mathfrak{g}}$-open sets of $\mathcal{T}_{\mathfrak{g}}: \mathcal{P}\left(\Omega_{\mu}\right) \longrightarrow \mathcal{P}\left(\Omega_{\mu}\right)$. With regards to the definition itself, an immediate consequence follows. By the relation $\mathcal{O}_{\mathfrak{g}, \sigma(\mu)}=\bigcup_{\nu \in I_{\sigma(\mu)}^{*}} \mathcal{O}_{\mathfrak{g}, \sigma(\nu, \mu)}$, is meant, for every $(\nu, \mu, \sigma(\mu), \sigma(\nu, \mu)) \in I_{\sigma(\mu)}^{*} \times I_{n}^{*} \times I_{\infty}^{*} \times I_{\infty}^{*}$, that $\mathcal{O}_{\mathfrak{g}, \sigma(\nu, \mu)} \in \mathcal{B}\left[\mathcal{T}_{\mathfrak{g}}\left(\Omega_{\mu}\right)\right]$ and $\mathcal{O}_{\mathfrak{g}, \sigma(\mu)} \in \mathcal{T}_{\mathfrak{g}}\left(\Omega_{\mu}\right)$ in the relation $\mathcal{O}_{\mathfrak{g}, \sigma(\mu)}=\bigcup_{\nu \in I_{\sigma(\mu)}^{*}} \mathcal{O}_{\mathfrak{g}, \sigma(\nu, \mu)}$, where $\mathcal{B}\left[\mathcal{T}_{\mathfrak{g}}\left(\Omega_{\mu}\right)\right]$ and $\mathcal{O}_{\mathfrak{g}, \sigma(\mu)} \in \mathcal{T}_{\mathfrak{g}}\left(\Omega_{\mu}\right)$ are given by

$$
\begin{aligned}
& \operatorname{proj}_{\alpha}: \bigotimes_{\mu \in I_{n}^{*}} \mathcal{B}\left[\mathcal{T}_{\mathfrak{g}}\left(\Omega_{\mu}\right)\right] \longrightarrow \mathcal{B}\left[\mathcal{T}_{\mathfrak{g}}\left(\Omega_{\alpha}\right)\right] \\
& \operatorname{proj}_{\alpha}: \bigotimes_{\mu \in I_{n}^{*}} \mathcal{T}_{\mathfrak{g}}\left(\Omega_{\mu}\right) \longrightarrow \mathcal{T}_{\mathfrak{g}}\left(\Omega_{\alpha}\right) \quad \forall \alpha \in I_{n}^{*}
\end{aligned}
$$

respectively. To this end, a Cartesian product topology (Cartesian $\mathcal{T}_{\mathfrak{g}}$-product) is one that having for $\mathcal{T}_{\mathfrak{g}}$-basis all $\mathcal{B}_{\mathcal{T}_{\mathfrak{g}}}$-open sets of the form $\operatorname{proj}_{\mu}^{-1}\left(\mathcal{O}_{\mathfrak{g}, \sigma(\nu, \mu)}\right)$, where $\mathcal{O}_{\mathfrak{g}, \sigma(\nu, \mu)} \in \mathcal{B}\left[\mathcal{T}_{\mathfrak{g}}\left(\Omega_{\mu}\right)\right]$ for every $(\nu, \mu, \sigma(\nu, \mu)) \in I_{\sigma(\mu)}^{*} \times I_{n}^{*} \times I_{\infty}^{*}$. Therefore, in order to define a Cartesian product $\mathcal{T}_{\mathfrak{g}}$-space, it suffices to take the above descriptions into account and postulate a proper definition on this ground. The following definition presents itself.

Definition 3.27. Let $\left\{\mathfrak{T}_{\mathfrak{g}}\left(\Omega_{\mu}\right):=\left(\Omega_{\mu}, \mathcal{T}_{\mathfrak{g}}\left(\Omega_{\mu}\right)\right): \mu \in I_{n}^{*}\right\}$ be a class of $n \geq 1 \mathcal{T}_{\mathfrak{g}}$-spaces and, for every $\mu \in I_{n}^{*}$, let $\mathcal{T}_{\mathfrak{g}, \Omega_{\mu}}: \mathcal{P}\left(\Omega_{\mu}\right) \longrightarrow \mathcal{P}\left(\Omega_{\mu}\right)$ be the $\mathfrak{g}$-topology for $\mathfrak{T}_{\mathfrak{g}}\left(\Omega_{\mu}\right)$. The Cartesian $\mathcal{T}_{\mathfrak{g}}$-product $:=\bigotimes_{\mu \in I_{n}^{*}} \mathcal{T}_{\mathfrak{g}}\left(\Omega_{\mu}\right)$ on the Cartesian product set $\Omega:=\bigotimes_{\mu \in I_{n}^{*}} \Omega_{\mu}$ is that having for $\mathcal{T}_{\mathfrak{g}}$-basis all $\mathcal{B}_{\mathcal{T}_{\mathfrak{g}}}$-open sets belonging to the following class:

$$
\mathcal{B}\left[\mathcal{T}_{\mathfrak{g}}(\Omega)\right]:=\left\{\operatorname{proj}_{\mu}^{-1}\left(\mathcal{O}_{\mathfrak{g}, \sigma(\nu, \mu)}\right): \mathcal{O}_{\mathfrak{g}, \sigma(\nu, \mu)} \in \mathcal{B}\left[\mathcal{T}_{\mathfrak{g}}\left(\Omega_{\mu}\right)\right] \forall(\nu, \mu, \sigma(\nu, \mu)) \in I_{\sigma(\mu)}^{*} \times I_{n}^{*} \times I_{\infty}^{*}\right\}
$$

The structure $\mathfrak{T}_{\mathfrak{g}}(\Omega):=\left(\Omega, \mathcal{T}_{\mathfrak{g}}(\Omega)\right)$ is called a "Cartesian product $\mathcal{T}_{\mathfrak{g}}$-space."

The fact that $\mathcal{O}_{\mathfrak{g}, \sigma(\nu, \mu)} \in \mathcal{B}\left[\mathcal{T}_{\mathfrak{g}}\left(\Omega_{\mu}\right)\right]$ and $\mathcal{O}_{\mathfrak{g}, \sigma(\mu)} \in \mathcal{T}_{\mathfrak{g}}\left(\Omega_{\mu}\right)$ hold for every $(\nu, \mu, \sigma(\mu), \sigma(\nu, \mu)) \in$ $I_{\sigma(\mu)}^{*} \times I_{n}^{*} \times I_{\infty}^{*} \times I_{\infty}^{*}$ makes it reasonable to write

$$
\begin{aligned}
\bigotimes_{\mu \in I_{n}^{*}} \mathcal{O}_{\mathfrak{g}, \sigma(\mu)} & \in \bigotimes_{\mu \in I_{n}^{*}} \mathcal{T}_{\mathfrak{g}}\left(\Omega_{\mu}\right) \\
\bigotimes_{\mu \in I_{n}^{*}}\left(\bigcup_{\nu \in I_{\sigma(\mu)}^{*}} \mathcal{O}_{\mathfrak{g}, \sigma(\nu, \mu)}\right) & =\bigcup_{\vec{\nu} \in \bigotimes_{\alpha \in I_{n}^{*} I_{\sigma(\alpha)}^{*}}\left(\bigotimes_{\alpha \in I_{n}^{*}} \mathcal{O}_{\mathfrak{g}, \sigma\left(\nu_{\alpha}, \alpha\right)}\right)} \\
& \in \bigotimes_{\mu \in I_{n}^{*}} \mathcal{B}\left[\mathcal{T}_{\mathfrak{g}}\left(\Omega_{\mu}\right)\right]
\end{aligned}
$$

where $\vec{\nu}:=\left(\nu_{1}, \nu_{2}, \ldots, \nu_{n}\right)$ and, for every $\alpha \in I_{n}^{*}, \nu_{\alpha} \in I_{\sigma(\alpha)}^{*}$. An immediate consequence of such relation is contained in the following lemma.

Lemma 3.28. If $\mathcal{T}_{\mathfrak{g}}: \mathcal{Q}(\Omega) \longrightarrow \mathcal{Q}(\Omega)$ is a one-valued map on the Cartesian product set $\Omega=$ $\bigotimes_{\mu \in I_{n}^{*}} \Omega_{\mu}$, where

$$
\mathcal{Q}(\Omega):=\left\{\mathcal{O}_{\mathfrak{g}, \sigma}=\bigcup_{\vec{\nu} \in \bigotimes_{\alpha \in I_{n}^{*} I_{\sigma(\alpha)}^{*}}}\left(\bigotimes_{\alpha \in I_{n}^{*}} \mathcal{O}_{\mathfrak{g}, \sigma\left(\nu_{\alpha}, \alpha\right)}\right): \quad \mathcal{O}_{\mathfrak{g}, \sigma} \in \bigotimes_{\mu \in I_{n}^{*}} \mathcal{B}\left[\mathcal{T}_{\mathfrak{g}}\left(\Omega_{\mu}\right)\right]\right\}
$$

then $\mathcal{T}_{\mathfrak{g}}: \mathcal{Q}(\Omega) \longrightarrow \mathcal{Q}(\Omega)$ is a $\mathfrak{g}$-topology on the Cartesian product set $\bigotimes_{\mu \in I_{n}^{*}} \Omega_{\mu}$.

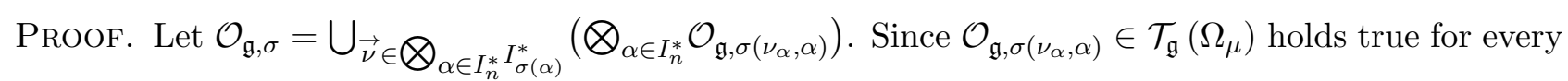
$\left(\nu_{\alpha}, \alpha, \sigma\left(\nu_{\alpha}, \alpha\right)\right) \in I_{\sigma(\alpha)}^{*} \times I_{n}^{*} \times I_{\infty}^{*}$, it is evident that $\mathcal{O}_{\mathfrak{g}, \sigma}=\emptyset$ only if, for every $\left(\nu_{\alpha}, \alpha, \sigma\left(\nu_{\alpha}, \alpha\right)\right) \in$ $I_{\sigma(\alpha)}^{*} \times I_{n}^{*} \times I_{\infty}^{*}, \mathcal{O}_{\mathfrak{g}, \sigma\left(\nu_{\alpha}, \alpha\right)}=\emptyset$. Thus, $\mathcal{T}_{\mathfrak{g}}(\emptyset)=\emptyset$.

Let $\mathcal{O}_{\mathfrak{g}, \sigma}=\bigcup_{\vec{\nu} \in \bigotimes_{\alpha \in I_{n}^{*} I_{\sigma(\alpha)}^{*}}}\left(\bigotimes_{\alpha \in I_{n}^{*}} \mathcal{O}_{\mathfrak{g}, \sigma\left(\nu_{\alpha}, \alpha\right)}\right)$. Then, since $\mathcal{Q}(\Omega) \subseteq \mathcal{Q}(\Omega)$, it follows that $\mathcal{O}_{\mathfrak{g}, \sigma}$ is a superset of $\mathcal{T}_{\mathfrak{g}}(\Omega)$. Thus, $\mathcal{T}_{\mathfrak{g}}\left(\mathcal{O}_{\mathfrak{g}, \sigma}\right) \subseteq \mathcal{O}_{\mathfrak{g}, \sigma}$. 
Let $\vec{\nu}=\left(\nu_{1}, \ldots, \nu_{n}\right)$ and $\vec{\kappa}=\left(\kappa_{1}, \ldots, \kappa_{n}\right)$, and consider

$$
\begin{aligned}
& \mathcal{O}_{\mathfrak{g}, \sigma}=\bigcup_{\vec{\nu} \in \bigotimes_{\alpha \in I_{n}^{*} I_{\sigma(\alpha)}^{*}}}\left(\bigotimes_{\alpha \in I_{n}^{*}} \mathcal{O}_{\mathfrak{g}, \sigma\left(\nu_{\alpha}, \alpha\right)}\right) \\
& \mathcal{O}_{\mathfrak{g}, \tau}=\bigcup_{\vec{\kappa} \in \bigotimes_{\beta \in I_{n}^{*} I_{\tau(\beta)}^{*}}}\left(\bigotimes_{\beta \in I_{n}^{*}} \mathcal{O}_{\mathfrak{g}, \tau\left(\kappa_{\beta}, \beta\right)}\right)
\end{aligned}
$$

Further, let us assume that $\vec{\eta}=\left(\nu_{1}, \ldots, \nu_{n}, \kappa_{1}, \ldots, \kappa_{n}\right), \mathbb{I}_{\sigma(\alpha)}^{*}:=\bigotimes_{\alpha \in I_{n}^{*}} I_{\sigma(\alpha)}^{*}$, and $\mathbb{I}_{\sigma(\beta)}^{*}:=\bigotimes_{\beta \in I_{n}^{*}} I_{\tau(\beta)}^{*}$. Then,

$$
\mathcal{O}_{\mathfrak{g}, \sigma} \cup \mathcal{O}_{\mathfrak{g}, \tau}=\bigcup_{\vec{\eta} \in \mathbb{I}_{\sigma(\alpha)}^{*} \times \mathbb{I}_{\sigma(\beta)}^{*}}\left(\bigotimes_{\mu \in I_{n}^{*}} \mathcal{O}_{\mathfrak{g}, \sigma\left(\nu_{\alpha}, \alpha\right)}\right) \cup\left(\bigotimes_{\beta \in I_{n}^{*}} \mathcal{O}_{\mathfrak{g}, \sigma\left(\kappa_{\beta}, \beta\right)}\right)
$$

Thus, $\mathcal{T}_{\mathfrak{g}}\left(\mathcal{O}_{\mathfrak{g}, \sigma} \cup \mathcal{O}_{\mathfrak{g}, \tau}\right) \subseteq \mathcal{T}_{\mathfrak{g}}\left(\mathcal{O}_{\mathfrak{g}, \sigma}\right) \cup \mathcal{T}_{\mathfrak{g}}\left(\mathcal{O}_{\mathfrak{g}, \tau}\right)$

Theorem 3.29. Let $\mathfrak{T}_{\mathfrak{g}, 1}(\Omega), \mathfrak{T}_{\mathfrak{g}, 2}(\Omega), \ldots, \mathfrak{T}_{\mathfrak{g}, n}(\Omega)$ be $n \geq 1 \mathcal{T}_{\mathfrak{g}}$-spaces and let $\mathfrak{T}_{\mathfrak{g}}(\Omega):=\bigotimes_{\nu \in I_{n}^{*}} \mathfrak{T}_{\mathfrak{g}, \nu}(\Omega)$ be the $\mathcal{T}_{\mathfrak{g}}$-space product. If the relation $\left(\mathcal{S}_{\mathfrak{g}, 1}, \ldots, \mathcal{S}_{\mathfrak{g}, n}\right) \in \bigotimes_{\nu \in I_{n}^{*}} \mathfrak{g}-\mathrm{S}\left[\mathfrak{T}_{\mathfrak{g}, \nu}\right]$ holds, then $\bigotimes_{\nu \in I_{n}^{*}} \mathcal{S}_{\mathfrak{g}, \nu} \in$ $\mathfrak{g}-\mathrm{S}\left[\bigotimes_{\nu \in I_{n}^{*}} \mathfrak{T}_{\mathfrak{g}, \nu}(\Omega)\right]$.

Proof. For every $\sigma \in I_{n}^{*}$, let

$$
\mathbf{o p}_{\mathfrak{g}, 12 \cdots \sigma}(\cdot)=\left(\operatorname{op}_{\mathfrak{g}, 12 \cdots \sigma}(\cdot), \neg \mathrm{op}_{\mathfrak{g}, 12 \cdots \sigma}(\cdot)\right) \in \mathcal{L}_{\mathfrak{g}, 12 \cdots \sigma}[\Omega]
$$

denotes the $\mathfrak{g}$-operator in $\bigotimes_{\nu \in I_{\sigma}^{*}} \mathfrak{T}_{\mathfrak{g}, \nu}(\Omega)$ and, for every $\nu \in I_{n}^{*}$, let $\left(\mathcal{S}_{\mathfrak{g}, \nu}, \mathcal{O}_{\mathfrak{g}, \nu}, \mathcal{K}_{\mathfrak{g}, \nu}\right) \in \mathfrak{g}-\mathrm{S}\left[\mathfrak{T}_{\mathfrak{g}, \nu}\right] \times$ $\mathcal{T}_{\mathfrak{g}, \nu} \times \neg \mathcal{T}_{\mathfrak{g}, \nu}$. Then,

$$
\begin{aligned}
\operatorname{op}_{\mathfrak{g}, 12 \cdots n}\left(\bigotimes_{\nu \in I_{n}^{*}} \mathcal{O}_{\mathfrak{g}, \nu}\right) & =\bigotimes_{\nu \in I_{n}^{*}} \mathrm{op}_{\mathfrak{g}, \nu}\left(\mathcal{O}_{\mathfrak{g}, \nu}\right) \\
\neg \mathrm{op}_{\mathfrak{g}, 12 \cdots n}\left(\bigotimes_{\nu \in I_{n}^{*}} \mathcal{K}_{\mathfrak{g}, \nu}\right) & =\bigotimes_{\nu \in I_{n}^{*}} \neg \mathrm{op}_{\mathfrak{g}, \nu}\left(\mathcal{K}_{\mathfrak{g}, \nu}\right)
\end{aligned}
$$

On the other hand, for every $\nu \in I_{n}^{*}$, the logical statement

$$
\left(\mathcal{S}_{\mathfrak{g}, \nu} \subseteq \mathrm{op}_{\mathfrak{g}, \nu}\left(\mathcal{O}_{\mathfrak{g}, \nu}\right)\right) \vee\left(\mathcal{S}_{\mathfrak{g}, \nu} \supseteq \neg \mathrm{op}_{\mathfrak{g}, \nu}\left(\mathcal{K}_{\mathfrak{g}, \nu}\right)\right)
$$

holds in $\mathfrak{T}_{\mathfrak{g}, \nu}$. Consequently,

$$
\begin{array}{lll} 
& \bigotimes_{\nu \in I_{n}^{*}}\left(\left(\mathcal{S}_{\mathfrak{g}, \nu} \subseteq \mathrm{op}_{\mathfrak{g}, \nu}\left(\mathcal{O}_{\mathfrak{g}, \nu}\right)\right) \vee\left(\mathcal{S}_{\mathfrak{g}, \nu} \supseteq \neg \mathrm{op}_{\mathfrak{g}, \nu}\left(\mathcal{O}_{\mathfrak{g}, \nu}\right)\right)\right) \\
\Rightarrow \quad & \left(\left(\bigotimes_{\nu \in I_{n}^{*}} \mathcal{S}_{\mathfrak{g}, \nu} \subseteq \bigotimes_{\nu \in I_{n}^{*}} \mathrm{op}_{\mathfrak{g}, \nu}\left(\mathcal{O}_{\mathfrak{g}, \nu}\right)\right) \vee\left(\bigotimes_{\nu \in I_{n}^{*}} \mathcal{S}_{\mathfrak{g}, \nu} \supseteq \bigotimes_{\nu \in I_{n}^{*}} \neg \mathrm{op}_{\mathfrak{g}, \nu}\left(\mathcal{K}_{\mathfrak{g}, \nu}\right)\right)\right) \\
\Rightarrow \quad & \left(\left(\bigotimes_{\nu \in I_{n}^{*}} \mathcal{S}_{\mathfrak{g}, \nu} \subseteq \mathrm{op}_{\mathfrak{g}, 12 \cdots n}\left(\bigotimes_{\nu \in I_{n}^{*}} \mathcal{O}_{\mathfrak{g}, \nu}\right)\right) \vee\left(\bigotimes_{\nu \in I_{n}^{*}} \mathcal{S}_{\mathfrak{g}, \nu} \supseteq \neg \mathrm{op}_{\mathfrak{g}, 12 \cdots n}\left(\bigotimes_{\nu \in I_{n}^{*}} \mathcal{K}_{\mathfrak{g}, \nu}\right)\right)\right)
\end{array}
$$

Therefore, the Boolean-valued functions

$$
\mathrm{P}_{\mathfrak{g}}\left(\bigotimes_{\nu \in I_{n}^{*}} \mathcal{S}_{\mathfrak{g}, \nu}, \bigotimes_{\nu \in I_{n}^{*}} \mathcal{O}_{\mathfrak{g}, \nu}, \bigotimes_{\nu \in I_{n}^{*}} \mathcal{K}_{\mathfrak{g}, \nu} ; \mathbf{o p}_{\mathfrak{g}, 12 \cdots n}(\cdot) ; \subseteq, \supseteq\right)
$$

holds on $\mathfrak{g}-\mathrm{S}\left[\mathfrak{T}_{\mathfrak{g}}\right] \times \mathcal{T}_{\mathfrak{g}} \times \neg \mathcal{T}_{\mathfrak{g}} \times \mathcal{L}_{\mathfrak{g}, 12 \cdots n}[\Omega] \times\{\subseteq, \supseteq\}$ and, hence, it follows that

$$
\bigotimes_{\nu \in I_{n}^{*}} \mathcal{S}_{\mathfrak{g}, \nu} \in \mathfrak{g}-\mathrm{G}\left[\bigotimes_{\nu \in I_{n}^{*}} \mathfrak{T}_{\mathfrak{g}, \nu}(\Omega)\right]
$$

The categorical classifications of $\mathfrak{T}$-sets and $\mathfrak{g}$-T-sets in the $\mathcal{T}$-space $\mathfrak{T} \subset \mathfrak{T}_{\mathfrak{g}}$ and, $\mathfrak{T}_{\mathfrak{g}}$-sets and $\mathfrak{g}$ - $\mathfrak{T}_{\mathfrak{g}}$-sets in the $\mathcal{T}_{\mathfrak{g}}$-space $\mathfrak{T}_{\mathfrak{g}}$ are discussed and diagrammed on this ground in the next sections. 


\section{Discussion}

\subsection{Categorical Classifications}

Having adopted a categorical approach in the classifications of $\mathfrak{g}-\mathfrak{T}_{\mathfrak{g}}$-sets in the $\mathcal{T}_{\mathfrak{g}}$-space $\mathfrak{T}_{\mathfrak{g}}$, the twofold purposes here are to establish the various relationships between the classes of $\mathfrak{T}_{\mathfrak{g}}$-open and $\mathfrak{T}_{\mathfrak{g}}$-closed sets and the classes of $\mathfrak{g}-\mathfrak{T}_{\mathfrak{g}}$-open and $\mathfrak{g}-\mathfrak{T}_{\mathfrak{g}}$-closed sets in the $\mathcal{T}_{\mathfrak{g}}$-space $\mathfrak{T}_{\mathfrak{g}}$, and to illustrate them through diagrams.

We have seen that, $\mathrm{S}\left[\mathfrak{T}_{\mathfrak{g}}\right] \subseteq \mathfrak{g}-\mathrm{S}\left[\mathfrak{T}_{\mathfrak{g}}\right]$. But, $\mathrm{S}\left[\mathfrak{T}_{\mathfrak{g}}\right]=\mathrm{O}\left[\mathfrak{T}_{\mathfrak{g}}\right] \cup \mathrm{K}\left[\mathfrak{T}_{\mathfrak{g}}\right]$ and $\mathfrak{g}-\mathrm{S}\left[\mathfrak{T}_{\mathfrak{g}}\right]=\mathfrak{g}-\mathrm{O}\left[\mathfrak{T}_{\mathfrak{g}}\right] \cup$ $\mathfrak{g}-\mathrm{K}\left[\mathfrak{T}_{\mathfrak{g}}\right]$. Consequently, $\mathrm{O}\left[\mathfrak{T}_{\mathfrak{g}}\right], \mathrm{K}\left[\mathfrak{T}_{\mathfrak{g}}\right] \subseteq \mathrm{S}\left[\mathfrak{T}_{\mathfrak{g}}\right]$ and $\mathfrak{g}-\mathrm{O}\left[\mathfrak{T}_{\mathfrak{g}}\right], \mathfrak{g}-\mathrm{K}\left[\mathfrak{T}_{\mathfrak{g}}\right] \subseteq \mathfrak{g}-\mathrm{S}\left[\mathfrak{T}_{\mathfrak{g}}\right] ; \mathrm{O}\left[\mathfrak{T}_{\mathfrak{g}}\right] \subseteq \mathfrak{g}-\mathrm{O}\left[\mathfrak{T}_{\mathfrak{g}}\right] \subseteq$ $\mathfrak{g}-\mathrm{S}\left[\mathfrak{T}_{\mathfrak{g}}\right]$ and $\mathrm{K}\left[\mathfrak{T}_{\mathfrak{g}}\right] \subseteq \mathfrak{g}-\mathrm{K}\left[\mathfrak{T}_{\mathfrak{g}}\right] \subseteq \mathfrak{g}-\mathrm{S}\left[\mathfrak{T}_{\mathfrak{g}}\right]$. In Figure 1, we present the relationships between the class $\mathrm{S}\left[\mathfrak{T}_{\mathfrak{g}}\right]=\mathrm{O}\left[\mathfrak{T}_{\mathfrak{g}}\right] \cup \mathrm{K}\left[\mathfrak{T}_{\mathfrak{g}}\right]$ of $\mathfrak{T}_{\mathfrak{g}}$-open and $\mathfrak{T}_{\mathfrak{g}}$-closed sets and the class $\mathfrak{g}-\mathrm{S}\left[\mathfrak{T}_{\mathfrak{g}}\right]=\mathfrak{g}-\mathrm{O}\left[\mathfrak{T}_{\mathfrak{g}}\right] \cup \mathfrak{g}-\mathrm{K}\left[\mathfrak{T}_{\mathfrak{g}}\right]$ of $\mathfrak{g}$ - $\mathfrak{T}_{\mathfrak{g}}$-open and $\mathfrak{g}$ - $\mathfrak{T}_{\mathfrak{g}}$-closed sets in the $\mathcal{T}_{\mathfrak{g}}$-space $\mathfrak{T}_{\mathfrak{g}}$.

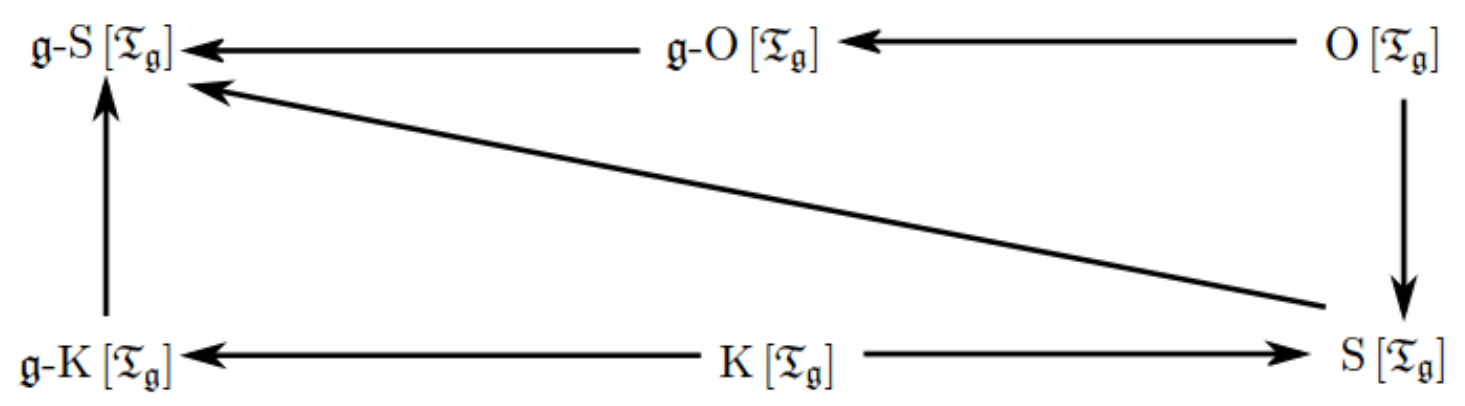

Fig. 1. Relationships: classes of $\mathfrak{T}_{\mathfrak{g}}$-sets and $\mathfrak{g}$ - $\mathfrak{T}_{\mathfrak{g}}$-sets

It is plain that $\mathfrak{g}-\nu-\mathrm{O}[\mathfrak{T}] \subseteq \mathfrak{g}-\mathrm{O}[\mathfrak{T}]$ and $\mathfrak{g}-\nu-\mathrm{O}[\mathfrak{T}] \subseteq \mathfrak{g}-\nu-\mathrm{O}\left[\mathfrak{T}_{\mathfrak{g}}\right] \subseteq \mathfrak{g}-\mathrm{O}\left[\mathfrak{T}_{\mathfrak{g}}\right]$ for every $\nu \in I_{3}^{0}$. Moreover, it is also clear that, $\mathfrak{g}-2-\mathrm{O}[\mathfrak{T}] \subseteq \mathfrak{g}-3-\mathrm{O}[\mathfrak{T}]$ and $\mathfrak{g}-0-\mathrm{O}[\mathfrak{T}] \subseteq \mathfrak{g}-1-\mathrm{O}[\mathfrak{T}] \subseteq \mathfrak{g}-3-\mathrm{O}[\mathfrak{T}]$, and $\mathfrak{g}-2-\mathrm{O}\left[\mathfrak{T}_{\mathfrak{g}}\right] \subseteq \mathfrak{g}-3-\mathrm{O}\left[\mathfrak{T}_{\mathfrak{g}}\right]$ and $\mathfrak{g}-0-\mathrm{O}\left[\mathfrak{T}_{\mathfrak{g}}\right] \subseteq \mathfrak{g}-1-\mathrm{O}\left[\mathfrak{T}_{\mathfrak{g}}\right] \subseteq \mathfrak{g}-3-\mathrm{O}\left[\mathfrak{T}_{\mathfrak{g}}\right]$. In fact, for every $\mathfrak{T}_{\mathfrak{g}}$-set $\mathcal{S}_{\mathfrak{g}} \subset \mathfrak{T}_{\mathfrak{g}}$ the relation $\operatorname{int}_{\mathfrak{g}}\left(\mathcal{S}_{\mathfrak{g}}\right) \subseteq \operatorname{cl}_{\mathfrak{g}} \circ \operatorname{int}_{\mathfrak{g}}\left(\mathcal{S}_{\mathfrak{g}}\right) \subseteq \operatorname{cl}_{\mathfrak{g}} \circ \operatorname{int}_{\mathfrak{g}} \circ \operatorname{cl}_{\mathfrak{g}}\left(\mathcal{S}_{\mathfrak{g}}\right) \supseteq \operatorname{int}_{\mathfrak{g}} \circ \operatorname{cl}_{\mathfrak{g}}\left(\mathcal{S}_{\mathfrak{g}}\right)$ holds. Consequently,

$$
\mathrm{op}_{\mathfrak{g}, 0}\left(\mathcal{S}_{\mathfrak{g}}\right) \subseteq \mathrm{op}_{\mathfrak{g}, 1}\left(\mathcal{S}_{\mathfrak{g}}\right) \subseteq \mathrm{op}_{\mathfrak{g}, 3}\left(\mathcal{S}_{\mathfrak{g}}\right) \supseteq \mathrm{op}_{\mathfrak{g}, 2}\left(\mathcal{S}_{\mathfrak{g}}\right) \quad \forall \mathcal{S}_{\mathfrak{g}} \subset \mathfrak{T}_{\mathfrak{g}}
$$

In Figure 2, we present the relationships between the class $\mathfrak{g}-\mathrm{O}\left[\mathfrak{T}_{\mathfrak{g}}\right]=\bigcup_{\nu \in I_{3}^{0}} \mathfrak{g}-\nu$-O$\left[\mathfrak{T}_{\mathfrak{g}}\right]$ of $\mathfrak{g}-\mathfrak{T}_{\mathfrak{g}}$-open sets of categories $0,1,2$ and 3 in the $\mathcal{T}_{\mathfrak{g}}$-space $\mathfrak{T}_{\mathfrak{g}}$, and the class $\mathfrak{g}$-O $[\mathfrak{T}]=\bigcup_{\nu \in I_{3}^{0}} \mathfrak{g}$ - $\nu$-O $[\mathfrak{T}]$ of $\mathfrak{g}$ - $\mathfrak{T}$-open sets of categories $0,1,2$ and 3 in the $\mathcal{T}$-space $\mathfrak{T} \subset \mathfrak{T}_{\mathfrak{g}}$.

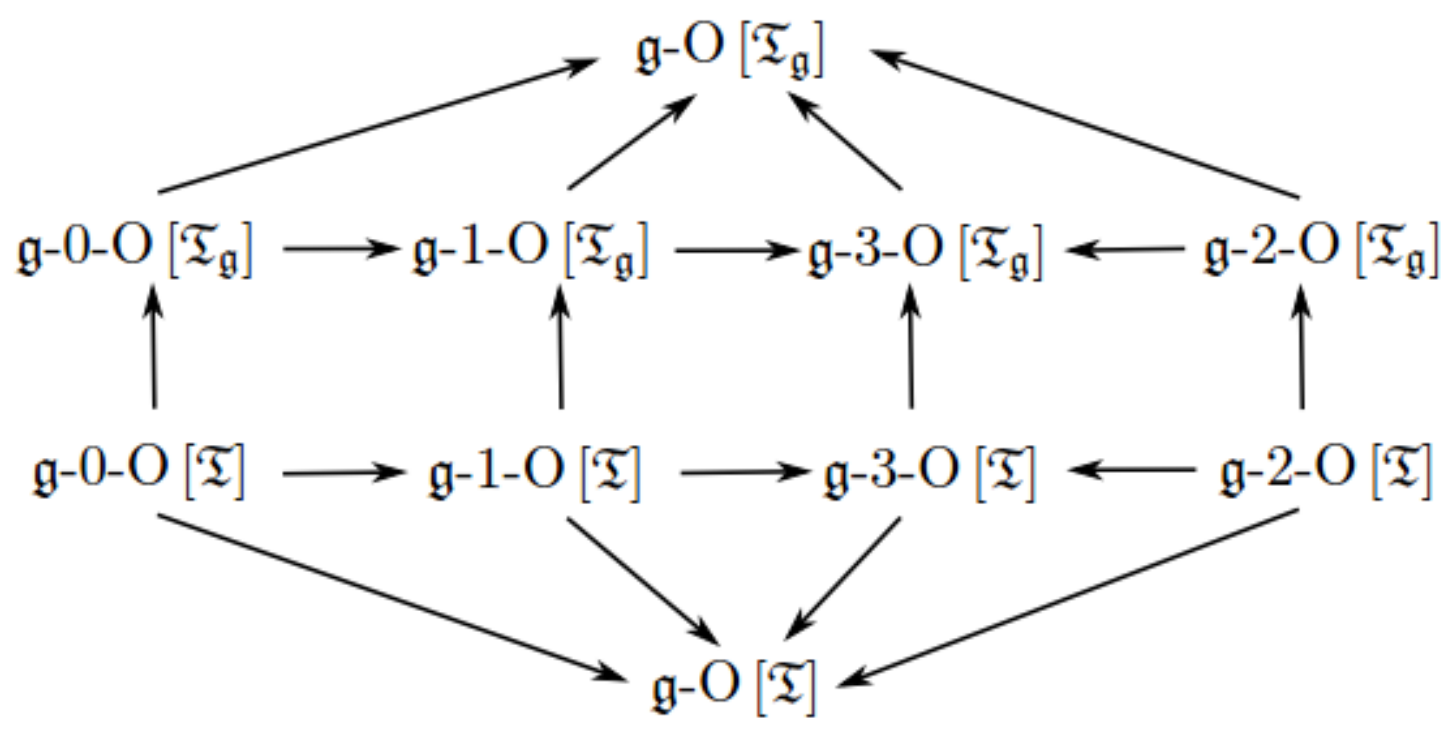

Fig. 2. Relationships: classes of $\mathfrak{g}$ - $\mathfrak{T}$-open sets and $\mathfrak{g}-\mathfrak{T}_{\mathfrak{g}}$-open sets 
It is plain that, $\mathfrak{g}-\nu-\mathrm{K}[\mathfrak{T}] \subseteq \mathfrak{g}-\mathrm{K}[\mathfrak{T}]$ and $\mathfrak{g}-\nu-\mathrm{K}[\mathfrak{T}] \subseteq \mathfrak{g}-\nu-\mathrm{K}\left[\mathfrak{T}_{\mathfrak{g}}\right] \subseteq \mathfrak{g}-\mathrm{K}\left[\mathfrak{T}_{\mathfrak{g}}\right]$ for every $\nu \in I_{3}^{0}$. Moreover, it is also clear that, $\mathfrak{g}-2-\mathrm{K}[\mathfrak{T}] \subseteq \mathfrak{g}-3-\mathrm{K}[\mathfrak{T}]$ and $\mathfrak{g}-0-\mathrm{K}[\mathfrak{T}] \subseteq \mathfrak{g}-1-\mathrm{K}[\mathfrak{T}] \subseteq \mathfrak{g}-3-\mathrm{K}[\mathfrak{T}]$, and $\mathfrak{g}-2-\mathrm{K}\left[\mathfrak{T}_{\mathfrak{g}}\right] \subseteq \mathfrak{g}-3-\mathrm{K}\left[\mathfrak{T}_{\mathfrak{g}}\right]$ and $\mathfrak{g}-0-\mathrm{K}\left[\mathfrak{T}_{\mathfrak{g}}\right] \subseteq \mathfrak{g}-1-\mathrm{K}\left[\mathfrak{T}_{\mathfrak{g}}\right] \subseteq \mathfrak{g}-3-\mathrm{K}\left[\mathfrak{T}_{\mathfrak{g}}\right]$. Because, for every $\mathfrak{T}_{\mathfrak{g}}$-set $\mathcal{S}_{\mathfrak{g}} \subset \mathfrak{T}_{\mathfrak{g}}$, the relations $\operatorname{cl}_{\mathfrak{g}}\left(\mathcal{S}_{\mathfrak{g}}\right) \supseteq \operatorname{int}_{\mathfrak{g}} \circ \operatorname{cl}_{\mathfrak{g}}\left(\mathcal{S}_{\mathfrak{g}}\right) \supseteq \operatorname{int}_{\mathfrak{g}} \circ \operatorname{cl}_{\mathfrak{g}} \circ \operatorname{int}_{\mathfrak{g}}\left(\mathcal{S}_{\mathfrak{g}}\right) \subseteq \operatorname{cl}_{\mathfrak{g}} \circ \operatorname{int}_{\mathfrak{g}}\left(\mathcal{S}_{\mathfrak{g}}\right)$ holds. Consequently,

$$
\neg \mathrm{op}_{\mathfrak{g}, 0}\left(\mathcal{S}_{\mathfrak{g}}\right) \supseteq \neg \mathrm{op}_{\mathfrak{g}, 1}\left(\mathcal{S}_{\mathfrak{g}}\right) \supseteq \neg \mathrm{op}_{\mathfrak{g}, 3}\left(\mathcal{S}_{\mathfrak{g}}\right) \subseteq \neg \mathrm{op}_{\mathfrak{g}, 2}\left(\mathcal{S}_{\mathfrak{g}}\right) \forall \mathcal{S}_{\mathfrak{g}} \subset \mathfrak{T}_{\mathfrak{g}}
$$

In Figure 3, we present the relations between the class $\mathfrak{g}-\mathrm{K}\left[\mathfrak{T}_{\mathfrak{g}}\right]=\bigcup_{\nu \in I_{3}^{0}} \mathfrak{g}-\nu-\mathrm{K}\left[\mathfrak{T}_{\mathfrak{g}}\right]$ of $\mathfrak{g}-\mathfrak{T}_{\mathfrak{g}}$-closed sets of categories $0,1,2$ and 3 in the $\mathcal{T}_{\mathfrak{g}}$-space $\mathfrak{T}_{\mathfrak{g}}$, and the class $\mathfrak{g}$-K $[\mathfrak{T}]=\bigcup_{\nu \in I_{3}^{0}} \mathfrak{g}-\nu$-K $[\mathfrak{T}]$ of $\mathfrak{g}$ - $\mathfrak{T}$-closed sets of categories $0,1,2$ and 3 in the $\mathcal{T}$-space $\mathfrak{T} \subset \mathfrak{T}_{\mathfrak{g}}$.

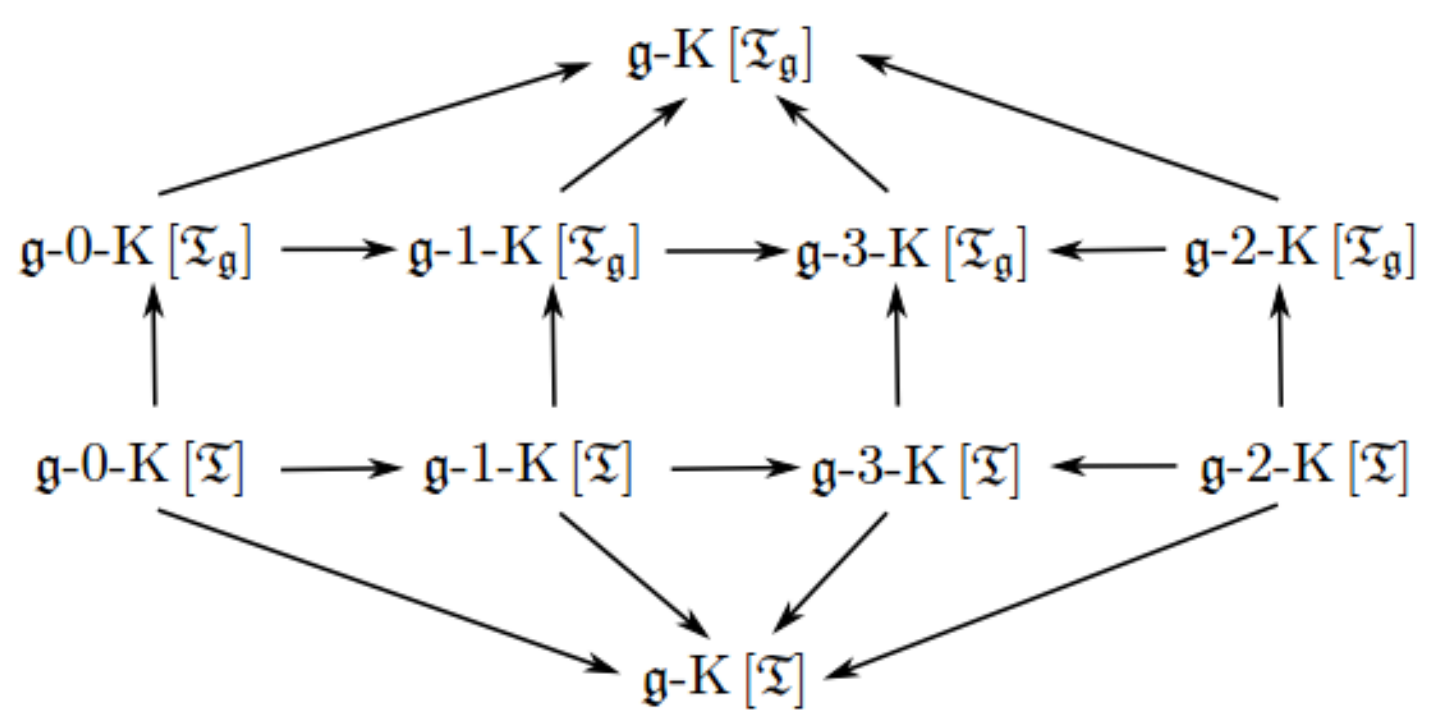

Fig. 3. Relationships: classes of $\mathfrak{g}$-T-closed sets and $\mathfrak{g}$ - $\mathfrak{T}_{\mathfrak{g}}$-closed sets

As in the papers of Caldas et al. [42], Dontchev [43], Jun et al. [8], and Tyagi et al. [6], among others, the manner we have positioned the arrows is solely to stress that, in general, none of the implications in FIGS 1, 2 and 3 is reversible.

At this stage, a nice application is worth considering, and is presented in the following section.

\subsection{A Nice Application}

Concentrating on fundamental concepts from the standpoint of the theory of $\mathfrak{g}-\mathfrak{T}_{\mathfrak{g}}$-sets, we shall now present a nice application. Let $\Omega=\left\{\xi_{\nu}: \nu \in I_{5}^{*}\right\}$ denotes the underlying set and consider the $\mathcal{T}_{\mathfrak{g}}$-space $\mathfrak{T}_{\mathfrak{g}}=\left(\Omega, \mathcal{T}_{\mathfrak{g}}\right)$, where

$$
\begin{aligned}
\mathcal{T}_{\mathfrak{g}}(\Omega) & =\left\{\emptyset,\left\{\xi_{1}\right\},\left\{\xi_{3}, \xi_{4}\right\},\left\{\xi_{1}, \xi_{3}, \xi_{4}\right\}\right\} \\
& =\left\{\mathcal{O}_{\mathfrak{g}, 1}, \mathcal{O}_{\mathfrak{g}, 2}, \mathcal{O}_{\mathfrak{g}, 3}, \mathcal{O}_{\mathfrak{g}, 4}\right\} \\
\neg \mathcal{T}_{\mathfrak{g}}(\Omega) & =\left\{\Omega,\left\{\xi_{2}, \xi_{3}, \xi_{4}, \xi_{5}\right\},\left\{\xi_{1}, \xi_{2}, \xi_{5}\right\},\left\{\xi_{2}, \xi_{5}\right\}\right\} \\
& =\left\{\mathcal{K}_{\mathfrak{g}, 1}, \mathcal{K}_{\mathfrak{g}, 2}, \mathcal{K}_{\mathfrak{g}, 3}, \mathcal{K}_{\mathfrak{g}, 4}\right\}
\end{aligned}
$$

respectively, stand for the classes of $\mathcal{T}_{\mathfrak{g}}$-open and $\mathcal{T}_{\mathfrak{g}}$-closed sets. Since conditions $\mathcal{T}_{\mathfrak{g}}(\emptyset)=\emptyset, \mathcal{T}_{\mathfrak{g}}\left(\mathcal{O}_{\mathfrak{g}, \nu}\right) \subseteq$ $\mathcal{O}_{\mathfrak{g}, \nu}$ for every $\nu \in I_{4}^{*}$, and $\mathcal{T}_{\mathfrak{g}}\left(\bigcup_{\nu \in I_{4}^{*}} \mathcal{O}_{\mathfrak{g}, \nu}\right)=\bigcup_{\nu \in I_{4}^{*}} \mathcal{T}_{\mathfrak{g}}\left(\mathcal{O}_{\mathfrak{g}, \nu}\right)$ are satisfied, it is clear that the onevalued map $\mathcal{T}_{\mathfrak{g}}: \mathcal{P}(\Omega) \rightarrow \mathcal{P}\left(\left\{\xi_{\nu}: \nu \in I_{5}^{*}\right\}\right)$ is a $\mathfrak{g}$-topology. Furthermore, it is easily checked that, $\mathcal{O}_{\mathfrak{g}, \mu} \in \mathfrak{g}-\nu-\mathrm{O}[\mathfrak{T}]$ for every $(\nu, \mu) \in I_{3}^{0} \times I_{4}^{*}$. Hence, the $\mathcal{T}_{\mathfrak{g}}$-open sets forming the $\mathfrak{g}$-topology $\mathcal{T}_{\mathfrak{g}}: \mathcal{P}(\Omega) \longrightarrow \mathcal{P}(\Omega)$ of the $\mathcal{T}_{\mathfrak{g}}$-space $\mathfrak{T}_{\mathfrak{g}}=\left(\Omega, \mathcal{T}_{\mathfrak{g}}\right)$ are $\mathfrak{g}$ - $\mathfrak{T}$-open sets relative to the $\mathcal{T}$-space $\mathfrak{T}=(\Omega, \mathcal{T})$. 
After calculations, the classes $\mathfrak{g}-\nu-\mathrm{O}\left[\mathfrak{T}_{\mathfrak{g}}\right]$ and $\mathfrak{g}-\nu$-K $\left[\mathfrak{T}_{\mathfrak{g}}\right]$, respectively, of $\mathfrak{g}$ - $\mathfrak{T}_{\mathfrak{g}}$-open and $\mathfrak{g}$ - $\mathfrak{T}_{\mathfrak{g}}$-closed sets of categories $\nu \in\{0,2\}$ then take the following forms:

$$
\begin{aligned}
\mathfrak{g}-\nu-\mathrm{O}\left[\mathfrak{T}_{\mathfrak{g}}\right]= & \mathcal{T}_{\mathfrak{g}} \cup\left\{\left\{\xi_{3}\right\},\left\{\xi_{4}\right\},\left\{\xi_{1}, \xi_{3}\right\},\left\{\xi_{1}, \xi_{4}\right\}\right\} \\
\mathfrak{g}-\nu-\mathrm{K}\left[\mathfrak{T}_{\mathfrak{g}}\right]=-\neg \mathcal{T}_{\mathfrak{g}} \cup\left\{\left\{\xi_{2}, \xi_{4}, \xi_{5}\right\},\left\{\xi_{1}, \xi_{2}, \xi_{3}, \xi_{5}\right\}\right. & \\
& \left.\left\{\xi_{1}, \xi_{2}, \xi_{4}, \xi_{5}\right\},\left\{\xi_{2}, \xi_{3}, \xi_{5}\right\}\right\}, \quad \forall \nu \in\{0,2\}
\end{aligned}
$$

On the other hand, those of categories $\nu \in\{1,3\}$ take the following forms:

$$
\begin{aligned}
\mathfrak{g}-\nu-\mathrm{O}\left[\mathfrak{T}_{\mathfrak{g}}\right] & =\mathcal{T}_{\mathfrak{g}} \cup\left\{\mathcal{O}_{\mathfrak{g}}: \mathcal{O}_{\mathfrak{g}} \in \mathcal{P}(\Omega) \backslash \mathcal{T}_{\mathfrak{g}}\right\} \\
\mathfrak{g}-\nu-\mathrm{K}\left[\mathfrak{T}_{\mathfrak{g}}\right] & =\neg \mathcal{T}_{\mathfrak{g}} \cup\left\{\mathcal{K}_{\mathfrak{g}}: \mathcal{K}_{\mathfrak{g}} \in \mathcal{P}(\Omega) \backslash \neg \mathcal{T}_{\mathfrak{g}}\right\}, \quad \forall \nu \in\{1,3\}
\end{aligned}
$$

The discussions carried out in the preceding sections can be easily verified from this nice application. The next section provides concluding remarks and future directions of the theory of $\mathfrak{g}$ - $\mathfrak{T}_{\mathfrak{g}}$-sets discussed in the preceding sections.

\section{Conclusion}

In this paper, we developed a new theory, called Theory of $\mathfrak{g}-\mathfrak{T}_{\mathfrak{g}}$-Sets. In its own rights, the proposed theory has several advantages. The very first advantage is that the theory holds equally well when $\left(\Omega, \mathcal{T}_{\mathfrak{g}}\right)=(\Omega, \mathcal{T})$ and other features adapted on this basis, in which case it might be called Theory of $\mathfrak{g}$-T-Sets. Hence, in a $\mathcal{T}_{\mathfrak{g}}$-space the theoretical framework categorises such pairs of concepts as $\mathfrak{g}$ - $\mathfrak{T}_{\mathfrak{g}}$-open and $\mathfrak{g}-\mathfrak{T}_{\mathfrak{g}}$-closed sets, $\mathfrak{g}$ - $\mathfrak{T}_{\mathfrak{g}}$-semi-open and $\mathfrak{g}$ - $\mathfrak{T}_{\mathfrak{g}}$-semi-closed sets, $\mathfrak{g}$ - $\mathfrak{T}_{\mathfrak{g}}$-pre-open and $\mathfrak{g}$ - $\mathfrak{T}_{\mathfrak{g}}$ pre-closed sets, and $\mathfrak{g}-\mathfrak{T}_{\mathfrak{g}}$-semi-pre-open and $\mathfrak{g}$ - $\mathfrak{T}_{\mathfrak{g}}$-semi-pre-closed sets as $\mathfrak{g}$ - $\mathfrak{T}_{\mathfrak{g}}$-sets of categories $0,1,2$, and 3, respectively, and theorises the concepts in a unified way; in a $\mathcal{T}$-space it categorises such pairs

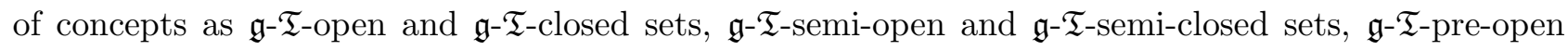

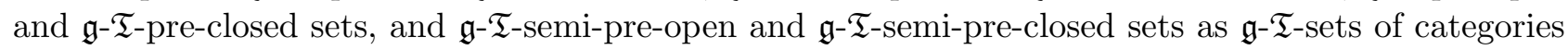
$0,1,2$, and 3 , respectively, and theorises the concepts in a unified way.

It is an interesting topic for future research to develop the theory of $\mathfrak{g}$ - $\mathfrak{T}_{\mathfrak{g}}$-sets of mixed categories. More precisely, for some pair $(\nu, \mu) \in I_{3}^{0} \times I_{3}^{0}$ such that $\nu \neq \mu$, to develop the theory of $\mathfrak{g}$ - $\mathfrak{T}_{\mathfrak{g}}$-open sets belonging to the class $\left\{\mathcal{O}_{\mathfrak{g}}=\mathcal{O}_{\mathfrak{g}, \nu} \cup \mathcal{O}_{\mathfrak{g}, \mu}:\left(\mathcal{O}_{\mathfrak{g}, \nu}, \mathcal{O}_{\mathfrak{g}, \mu}\right) \in \mathfrak{g}-\nu-\mathrm{O}\left[\mathfrak{T}_{\mathfrak{g}}\right] \times \mathfrak{g}-\mu-\mathrm{O}\left[\mathfrak{T}_{\mathfrak{g}}\right]\right\}$ and the theory of $\mathfrak{g}$ - $\mathfrak{T}_{\mathfrak{g}}$-closed sets belonging to the class $\left\{\mathcal{K}_{\mathfrak{g}}=\mathcal{K}_{\mathfrak{g}, \nu} \cup \mathcal{K}_{\mathfrak{g}, \mu}:\left(\mathcal{K}_{\mathfrak{g}, \nu}, \mathcal{K}_{\mathfrak{g}, \mu}\right) \in \mathfrak{g}-\nu-\mathrm{K}\left[\mathfrak{T}_{\mathfrak{g}}\right] \times \mathfrak{g}-\mu-\mathrm{K}\left[\mathfrak{T}_{\mathfrak{g}}\right]\right\}$ in a $\mathcal{T}_{\mathfrak{g}}$-space $\mathfrak{T}_{\mathfrak{g}}$, as Andrijević [22] and Caldas et al. [44] developed the theory of $b$-open and $b$-closed sets in a $\mathcal{T}$-space $\mathfrak{T}$. Such two theories are what we thought would certainly be worth considering, and the discussion of this work ends here.

\section{Author Contributions}

All the authors contributed equally to this work. They all read and approved the last version of the manuscript.

\section{Conflicts of Interest}

The authors declare no conflict of interest.

\section{Acknowledgement}

The authors would like to express their sincere thanks to Prof. (Dr.) F. William Lawvere (Professor Emeritus and inventor of Lawvere-Tierney Topology) for motivating them to develop the subject matter and, Prof. (Dr.) Endre Makai, Jr. (Professor Emeritus of the Mathematical Institute of the Hungarian Academy of Sciences) for his valuable suggestions. 


\section{References}

[1] S. Ersoy, M. Bilgin, İ. İnce, Generalized Closed Set in Topological Spaces, Mathematica Moravica 19(1) (2015) 49-56.

[2] S. Al Ghour, W. Zareer, Omega Open Sets in Generalized Topological Spaces, Journal of Nonlinear Sciences and Applications 9 (2016) 3010-3017.

[3] P. Jeyanthi, P. Nalayini, M. Mocanu, g* $\lambda_{\mu}$-Closed Sets and Generalized Topological Spaces, Boletim da Sociedade Paranaense de Matemática 34(1) (2016) 203-212.

[4] I. Reilly, Generalized Closed Sets: A Survey of Recent Works, General and Geometric Topology and its Applications 1248 (2002) 1-11.

[5] D. Saravanakumar, N. Kalaivani, G. S. S. Krishnan, On $\tilde{\mu}$-Open Sets in Generalized Topological Spaces, Malaya Journal of Matematik 3(3) (2015) 268-276.

[6] B. K. Tyagi, Harsh V. S. Chauhan, On Generalized Closed Sets in a Generalized Topological Spaces, CUBO A Mathematical Journal 18(01) (2016) 27-45.

[7] A. Danabalan, C. Santhi, A Class of Separation Axioms in Generalized Topology, Mathematical Journal of Interdisciplinary Sciences 4(2) (2016) 151-159.

[8] Y. B. Jun, S. W. Jeong, H. J. Lee, J. W. Lee, Applications of Pre-Open Sets, Applied General Topology, Universidad Politécnica de Valencia 9(2) (2008) 213-228.

[9] V. Pavlović, A. S. Cvetković, On Generalized Topologies arising from Mappings, Vesnik, Universidad Politécnica de Valencia 38(3) (2012) 553-565.

[10] O. Njåstad, On Some Classes of Nearly Open Sets, Pacific Journal of Mathematics 15(3) (1965) 961-970.

[11] D. Andrijević, Semi-Pre-open Sets, Matematički Vesnik 38(1) (1986) 24-32.

[12] H. Ogata, Operations on Topological Spaces and Associated Topology, Mathematica Japonica 36 (1991) $175-184$.

[13] P. Jeyanthi, P. Nalayini, T. Noiri, $\Delta_{\mu}$-Sets and $\nabla_{\mu}$-Sets in Generalized Topological Spaces, Georgian Mathematical Journal 24(3) (2016) 403-407.

[14] Á. Császár, Generalized Topology, Generalized Continuity, Acta Mathematica Hungarica 96(4) (2002) 351-357.

[15] N. Levine, Generalized Closed Set in Topological Spaces, Rendiconti del Circolo Matematico di Palermo 19 (1970) 89-96.

[16] N. Levine, Semi-Open Sets and Semi-Continuity in Topological Spaces, American Mathematical Monthly 70 (1963) 19-41.

[17] Á. Császár, Remarks on Quasi-Topologies, Acta Mathematica Hungarica 119(1-2) (2008) 197-200.

[18] Á. Császár, Further Remarks on the Formula for $\gamma$-Interior, Acta Mathematica Hungarica 113(4) (2006) 325-332.

[19] Á. Császár, Generalized Open Sets in Generalized Topologies, Acta Mathematica Hungarica 106(12) (2005) 53-66.

[20] Á. Császár, Generalized Open Sets, Acta Mathematica Hungarica 75(1-2) (1997) 65-87. 
[21] K. Rajeshwari, T. D. Rayanagoudar, S. M. Patil, On Semi Generalized $\omega \alpha$-Closed Sets in Topological Spaces, Global Journal of Pure and Applied Mathematics 13(9) (2017) 5491-5503.

[22] D. Andrijević, On b-Open Sets, Matematički Vesnik 48 (1996) 59-64.

[23] S. Bayhan, A. Kanibir, I. L. Reilly, On Functions between Generalized Topological Spaces, Applied General Topology 14(2) (2013) 195-203.

[24] P. Bhattacharyya, B.K. Lahiri, Semi-Generalized Closed Sets in Topology, Indian Journal of Mathematics 29 (1987) 376-382.

[25] C. Boonpok, On Generalized Continuous Maps in Čech Closure Spaces, General Mathematics 19(3) (2011) 376-382.

[26] J. Cao, M. Ganster, I. Reilly, On Generalized Closed sets, Topology and its Applications 123(1) (2002) 37-46.

[27] J. Dontchev, T. Noiri, Quasi-Normal Spaces and $\pi$ g-Closed Sets, Acta Mathematica Hungarica 89(3) (2000) 211-219.

[28] J. Dontchev, On Generalizing Semi-Pre-open Sets, Memoirs of the Faculty of the Science, Kochi University Series A Mathematics 16 (1995) 35-48.

[29] Y. Gnanambal, On Generalized Preregular Closed Sets in Topological Spaces, Indian Journal of Pure and Applied Mathematics 28 (1997) 351-360.

[30] A. Gupta, R. D. Sarma, A Note on some Generalized Closure and Interior Operators in a Topological Space, Mathematics for Applications 6 (2017) 11-20.

[31] R. A. Hosny, D. Al-Kadi, Types of Generalized Sets with Ideal, International Journal of Computer Applications 80(4) (2013) 11-14.

[32] M. K. R. S. Veera Kumar, Between Closed Sets and g-Closed Sets, Memoirs of the Faculty of the Science, Kochi University Series A Mathematics 21 (2000) 1-19.

[33] L. L. L. Lusanta, H. M. Rara, Generalized Star $\alpha$-b-Separation Axioms in Bigeneralized Topological Spaces, Applied Mathematical Sciences 9(75) (2015) 3725-3737.

[34] H. Maki, R. Devi, K. Balachandran, Associated Topologies of Generalized $\alpha$-Closed Sets and $\alpha$-Generalized Closed Sets, Memoirs of the Faculty of the Science, Kochi University Series A Mathematics 15 (1994) 51-63.

[35] H. Maki, R. Devi, K. Balachandran, Generalized $\alpha$-Closed Sets in Topology, Bulletin of Fukuoka University of Education Part III 42 (1993) 13-21.

[36] A. S. Mashhour, I. A. Hasanein, S. N. E. Deeb, $\alpha$-Continuous and $\alpha$-Open Mappings, Acta Mathematica Hungarica 41(3-4) (1983) 213-218.

[37] B. Roy, On a Type of Generalized Open Sets, Applied General Topology 12(2) (2011) 163-173.

[38] P. Sundaram, M. Sheik John, On w-Closed Sets in Topology, Acta Ciencia Indica 4 (2000) 389392.

[39] M. I. Khodabocus, A Generalized Topological Space endowed with Generalized Topologies, PhD Dissertation, University of Mauritius (2020) Réduit, Mauritius.

[40] M. S. Sarsak, On some Properties of Generalized Open Sets in Generalized Topological Spaces, Demonstratio Mathematica XLVI (2) (2013) 415-427. 
[41] T. Noiri, Unified Characterizations for Modifications of $\mathrm{R}_{0}$ and $\mathrm{R}_{1}$ Topological Spaces, Circolo Matematico di Palermo 55(2) (2006) 29-42.

[42] M. Caldas, S. Jafari, R. K. Saraf, Semi- - -Open Sets and New Classes of Maps, Bulletin of the Iranian Mathematical Society 31(2) (2005) 37-52.

[43] J. Dontchev, On Some Separation Axioms Associated with the $\alpha$-Topology, Memoirs of the Faculty of the Science, Kochi University Series A Mathematics 18 (1997) 31-35.

[44] M. Caldas, S. Jafari, On Some Applications of b-Open Sets in Topological Spaces, Kochi Journal of Mathematics 2 (2007) 11-19. 